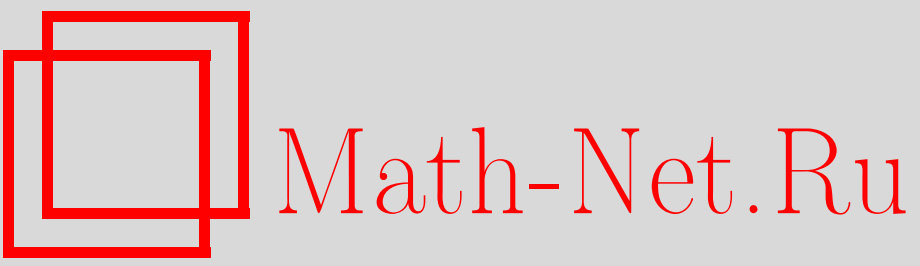

Е. И. Динабург, Некоторые вопросы спектральной теории дискретных операторов с квазипериодическими коэффициентами, УМН, 1997, том 52, выпуск 3, 3-52

DOI: https://doi.org/10.4213/rm848

Использование Общероссийского математического портала Math-Net.Ru подразумевает, что вы прочитали и согласны с пользовательским соглашением

http://www.mathnet.ru/rus/agreement

Параметры загрузки:

IP: 52.23 .180 .231

26 апреля 2023 г., 15:36:07 


\title{
НЕКОТОРЫЕ ВОПРОСЫ СПЕКТРАЛЬНОЙ ТЕОРИИ ДИСКРЕТНЫХ ОПЕРАТОРОВ \\ С КВАЗИПЕРИОДИЧЕСКИМИ КОЭФФИЦИЕНТАМИ
}

\author{
Е. И. ДинАБУРГ
}

СОДЕРЖАНИЕ

Введение

Часть первая. Одномерные операторы Шрёдингера с квазипериодичес-

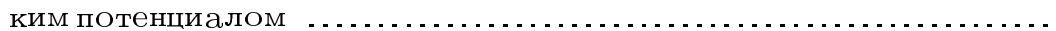

$\S 1$. Локализация Андерсона для одномерного разностного оператора Шрёдингера с квазипериодическим потенциалом .............. 8

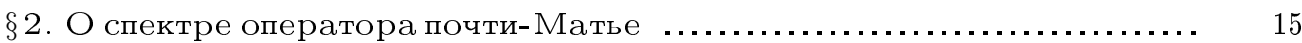

Дополнение к части первой. О непрерывном одномерном уравнении Шрёдингера с квазипериодическим потенциалом ................ .

Часть вторая. Экспоненциальная локализация собственных функций квазипериодических операторов с дальнодействием на решетке . 21

$\S 1$. Постановка задачи. Формулировка результата ................. 21

$\S 2$. Доказательство теоремы ... . . . . . . . . . . . . . . . . . . . . . . . . . . . 23

$\S 3$. Доказательство индуктивной леммы . . . . . . . . . . . . . . . . . . . . . 32

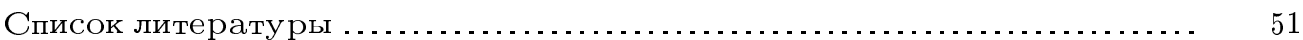

\section{Введение}

Эта статья посвящается Якову Григорьевичу Синаю. Он внес важньй вклад в разработку методов анализа спектральных свойств операторов с квазипериодическими коэф фициентами и получил результаты общего характера о структуре спектра и собственных функций таких операторов (см. [1]-[4], а также [5]).

Основньм объектом изучения являются не индивидуальные операторы, а эргодические семейства операторов, зависяшие от параметра, значения которого пробегают фазовое пространство некоторой эргодической динамической системы, а коэффициенты индивидуального оператора, входящего в семейство, определяются значениями функций вдоль траектории динамической системы. Подавляющее число результатов о спектральных свойствах таких семейств носит метрический характер и относится

Работа выполнена при частичной финансовой поддержке Российского фонда фундаментальных исследований (грант № 93-01-16090 и грант № 95-01-00632a). 
к почти всем (по инвариантной мере динамической системы), т.е. типичным, значениям параметра.

Для рассматриваемых в этой статье семейств операторов общая постановка задачи может быть сформулирована следующим образом.

Пусть $\mathbb{Z}^{\nu}-\nu$-мерная целочисленная решетка в $\mathbb{R}^{\nu}(\nu \geqslant 1), n=\left(n_{1}, \ldots, n_{\nu}\right) \in \mathbb{Z}^{\nu}$, $|n|=\sum_{i=1}^{\nu}\left|n_{i}\right|$.

Мы рассматриваем динамические системы с $\nu$-мерным дискретным временем, $\left(X,\left\{T^{n}\right\}, \mu\right)$, где $X$ - измеримое пространство, $\left\{T^{n}\right\}$ - измеримое действие групшы $\mathbb{Z}^{\nu}$ на $X$, сохраняющее меру $\mu, \mu(X)=1$.

Пусть $\varphi(n, x), x \in X, n \in \mathbb{Z}^{\nu}$, - функщия двух переменных, принадлежашая при любом фиксированном $n$ пространству $L^{\infty}(X, \mu)$. При любом $\rho \geqslant 0$ рассмотрим множество функций $\varphi(n, x)$, для которых

$$
\|\varphi\|_{\rho}=\|\varphi(n, x)\|_{\rho}=\sum_{n \in \mathbb{Z}^{\nu}}\|\varphi(n, x)\|_{\infty} e^{\rho|n|}<\infty
$$

Банахово пространство таких функций обозначим $B^{\rho}$.

Определим на $B^{\rho}$ умножение по формуле:

$$
(\varphi \psi)(n, x)=\sum_{m \in \mathbb{Z}^{\nu}} \varphi\left(n-m, T^{m} x\right) \psi(m, x)
$$

и инволющию

$$
(\varphi(n, x))^{*}=\overline{\varphi\left(-n, T^{n} x\right)} .
$$

Здесь $\varphi(n, x), \psi(n, x) \in B^{\rho}$, черта означает комплексное сопряжение.

Легко видеть, что $\|\varphi \psi\|_{\rho} \leqslant\|\varphi\|_{\rho}\|\psi\|_{\rho}$ и $\left\|\varphi^{*}\right\|_{\rho}=\|\varphi\|_{\rho}$.

Пространство $B^{\rho}$ с введенными операциями инволюции и умножения становится *-алгеброй. Для того, чтобы подчеркнуть его алгебраическую структуру, мы будем в дальнейшем пользоваться вместо $B^{\rho}$ обозначением $A^{\rho}$.

Ясно, что $A^{\rho} \subset A^{\rho_{1}}$ при $\rho_{1} \leqslant \rho$.

Введем важное для дальнейшего $*$-представление алгебры $A^{\rho}$ в алгебру семейств ограниченных операторов в $\ell^{2}\left(\mathbb{Z}^{\nu}\right)$, зависящих от параметра $x \in X$.

Каждому элементу $\varphi(n, x)$ алгебры $A^{\rho}$ поставим в соответствие семейство операторов $\widehat{\varphi}(x)$, действуюших в $\ell^{2}\left(\mathbb{Z}^{\nu}\right)$. Его матричное представление в естественном базисе $\left\{e_{n}\right\}=\left\{\delta_{n m}\right\}, n, m \in \mathbb{Z}^{\nu}$, имеет вид

$$
\widehat{\varphi}_{m n}(x)=\varphi\left(n-m, T^{m} x\right) .
$$

Оператор $\widehat{\varphi}(x)$ корректно определен для почти всех $x$ и представляет собой ограниченньй оператор в $\ell^{2}\left(\mathbb{Z}^{\nu}\right)$, поскольку его матричные элементы экспоненциально быстро убьвают при $|n-m| \rightarrow \infty$.

Мы будем говорить, что семейство операторов $\widehat{\varphi}(x)$ определяется функцией $\varphi(n, x)$ и ассощиировано с динамической системой $\left(X,\left\{T^{n}\right\}, \mu\right)$. Если $\left(X,\left\{T^{n}\right\}, \mu\right)$ - эргодическая система, то семейство $\widehat{\varphi}(x)$ называется эргодическим семейством.

Очевидно, что если $\widehat{\varphi}(x)$ и $\widehat{\psi}(x)$ - два семейства операторов, определяемых функциями $\varphi(n, x)$ и $\psi(n, x)$, соответственно, и ассоциированных с одной и той же динамической системой, то семейство $\widehat{\eta}(x)=\widehat{\varphi} \widehat{\psi}(x)$ определяется элементом 
$\eta(n, x)=(\varphi \psi)(n, x)$ алгебры $A^{\rho}$. Кроме того, непосредственная проверкапоказывает, что условие $\varphi^{*}(n, x)=\varphi(n, x)$ является необходимым и достаточным условием самосопряженности операторов $\widehat{\varphi}(x)$.

ЗАмЕчАниЕ 1 . Семейство $\widehat{\varphi}(x)$ можно рассматривать как оператор со случайными коэффициентами. Случайность определяется случайным выбором точки $x \in X$, распределение вероятностей которой определяется мерой $\mu$.

ЗАмечАниЕ 2 . Л.А. Пастуром было показано, что если $\widehat{\varphi}(x)$ - эргодическое семейство операторов, то спектр $\sigma(x)$ и его абсолютно непрерьвная $\sigma_{a c}(x)$, сингулярно непрерьвная $\sigma_{s c}(x)$ и точечная $\sigma_{p p}(x)$ (замыкание множества собственных значений) компоненты для почти всех $x$ не зависят от $x$ (см., например, $[6]$ ).

ЗАмечАниЕ 3. Класс операторов, определенньй выше, включает в себя разностньй оператор Шрёдингера со случайным потенщиалом

$$
\Delta+U_{x}(n),
$$

где $(\Delta \psi)(n)=\sum_{m:|n-m|=1} \psi(m)$, потенциал $U_{x}(n)=U\left(T^{n} x\right)$. В самом деле, достаточно положить $\varphi(n, x)=1$ при $|n|=1, \varphi(0, x)=U(x), \varphi(n, x)=0$ при $|n|>1$.

Опишем некоторые примеры операторов Шрёдингера и связанных с ними семейств операторов, ассоциированных с различными динамическими системами.

\section{а) Модель Андерсона.}

Рассмотрим динамическую систему $\left(X,\left\{T^{n}\right\}, \mu\right)$, где $X=\prod_{n \in \mathbb{Z}^{\nu}} X_{n}$ - произведение изоморфных пространств $X_{n} \approx \mathbb{R}, x=\left\{x_{i}\right\}_{i \in \mathbb{Z}^{\nu}}, x_{i} \in X_{i}, \mu=\prod_{i \in \mathbb{Z}^{\nu}} \nu_{i}, \nu_{j}$ - мера на $X_{j}, \nu_{i}=\eta, T^{n} x=\left\{x_{i-n}\right\}$. Положим $U(x)=x_{0}(x)$, где $x_{0}(x)$-нулевая координата точки $x$.

Иными словами, при фиксированном $x$ значение потенциала $U\left(T^{n} x\right)$ есть реализация последовательности независимых одинаково распределенных (с распределением $\eta$ ) случайных величин. Такая модель назьвается моделью Андерсона.

Известно (см., например, [6]), что для $\nu=1$ и $\eta$, имеюших ограниченную плотность с компактньм носителем, оператор $\widehat{\varphi}(x)$ имеет чисто точечньй спектр для почти всех $x$ по мере $\mu$ и его собственные функции экспоненциально убывают при $|n| \rightarrow \infty$.

Принято говорить, что для семейства операторов $\widehat{\varphi}(x)$ имеет место локализация Андерсона, если для почти всех $x$ оператор $\widehat{\varphi}(x)$ имеет чисто точечньй спектр, и экспоненциальная локализация Андерсона, если при этом его собственные функции экспоненциально убьвают.

Экспоненциальная локализация Андерсона для оператора Шрёдингера со случайным потенциалом имеет место для широкого класса операторов.

В этой статье мы будем изучать условия существования чисто точечного спектра для семейств операторов, ассоциированных с динамическими системами с чисто точечным спектром, которые приводят к операторам с почти периодическими коэффишиентами.

Читателя, интересующегося операторами со случайньм потенциалом типа модели Андерсона, мы отсылаем к монографии [6], содержащей все основные результаты, полученные до 1990 года включительно, а по поводу более поздних результатов к статье [7]. 
б) Оператор Шрёдингера, ассоциированный с алгебраическим автоморфизмом тора Tor $^{2}$.

Пусть $X=$ Tor $^{2}=\mathbb{R}^{2} / \mathbb{Z}^{2}, x=\left(x_{1}, x_{2}\right) \in X, 0 \leqslant x_{1}<1,0 \leqslant x_{2}<1$, $A=\left(\begin{array}{ll}a & b \\ c & d\end{array}\right)$ - целочисленная матрица, $\operatorname{det} A=1, \operatorname{tr} A>2$. Хорошо известно (см., например, [8]), что $\left(X,\left\{T^{n}\right\}, \mu\right), n \in \mathbb{Z}^{1},-$ эргодическая динамическая система, где $T x=\left(a x_{1}+b x_{2}, c x_{1}+d x_{2}\right) \bmod 1, \mu$-мера Лебега $d x_{1} d x_{2}$. Более того, динамическая система $\left(X,\left\{T^{n}\right\}, \mu\right)$ обладает перемешиванием всех степеней, имеет положительную энтропию Колмогорова-Синая, является $K$-системой и т. п. (см. [8]). Для произвольной гладкой функции $U(x)$ на $X$ со средним 0 рассмотрим эргодическое семейство операторов в $\ell^{2}\left(\mathbb{Z}^{1}\right)$ :

$$
(\widehat{\varphi}(x) f)(n)=f(n+1)+f(n-1)+g U\left(T^{n} x\right) f(n),
$$

где $f(n) \in \ell^{2}\left(\mathbb{Z}^{1}\right), n \in \mathbb{Z}^{1}, g>0$ - параметр. Единственный результат, доказанньй к настояшему времени для этого случая, состоит в том, что для достаточно малых $g$ и для $g^{1 / 2}<|E|<2-g^{1 / 2}$ показатель Ляпунова $\gamma(E)$ положителен для широкого класса функции $U(x)($ см. $[9])$.

Напомним определение показателя Ляпунова.

Положим

$$
\Psi(n)=\left(\begin{array}{c}
\psi(n+1) \\
\psi(n)
\end{array}\right), \quad U_{n}(x)=U\left(T^{n} x\right) .
$$

Уравнение $\psi(n+1)+\psi(n-1)+U_{n}(x) \psi(n)=E \psi(n)$ может быть переписано в матричной форме:

$$
\Psi(n+1)=T_{n}^{x}(E) \Psi(n),
$$

где

$$
T_{n}^{x}(E)=\left(\begin{array}{cr}
E-U_{n}(x) & -1 \\
1 & 0
\end{array}\right)
$$

есть матрища 2-го порядка. Тогда

$$
\Psi(n)=A_{n}^{x}(E) \Psi(0)=T_{n}^{x}(E) T_{n-1}^{x}(E) \cdots T_{1}^{x}(E) \Psi(0) .
$$

Обозначим

$$
\gamma(E)=\lim _{n \rightarrow \infty} \frac{1}{n} \ln \left\|A_{n}^{x}(E)\right\|,
$$

где $\|$ • ||-операторная норма в множестве матриц 2-го порядка. По теореме Фюрстенберга (см. [6]) при каждом $E$ этот предел сушествует для всех $E$ и для почти всех $x$ и не зависит от $x$. Этот общий предел и назьвается показателем Ляпунова. Данное определение относится только к одномерному случаю.

в) Эргодические семейства операторов с квазипериодическими коэффициентами.

в1) Мерилендская модель.

Пусть $\left(X,\left\{T^{n}\right\}, \mu\right)=\left(S^{1},\left\{T_{\omega}^{n}\right\}, \mu\right)$ - динамическая система, где $T_{\omega}$ - поворот единичной окружности $S^{1}$ на иррациональньй угол $\omega, \mu$-мера Лебега на $S^{1}$. Рассмотрим семейство операторов $\widehat{\varphi}_{M}(x)$ в $\ell^{2}\left(\mathbb{Z}^{1}\right)$ :

$$
\left(\widehat{\varphi}_{M}(x) f\right)(n)=f(n+1)+f(n-1)+\lambda \operatorname{tg}(\pi(x+n \omega)) f(n) .
$$

Здесь $\lambda$ - действительньй параметр. 
Формально это семейство операторов не содержится в алгебре $A^{\rho}$ ни при каком $\rho$, но тем не менее такое семейство операторов может быть изучено. Для него известно (см. [10], [11]), что для всех $\lambda$ и иррациональных $\omega$, принадлежаших множеству полной меры, спектр оператора $\widehat{\varphi}_{M}(x)$ при всех $x$ чисто точечный, а его собственные функции экспоненциально убьвают. При доказательстве этого результата существенно используется вид потенциала.

в2) Обобщенная мерилендская модель.

Пусть $\left(X,\left\{T^{n}\right\}, \mu\right)$ - та же динамическая система, что и в предыдущем примере. Рассмотрим произвольньй элемент $\varphi(n, x) \in A^{\rho}$ при некотором $\rho>0$ и семейство операторов $\widehat{\varphi}_{G M}(x)$, которое имеет вид

$$
\left(\widehat{\varphi}_{G M}(x) f\right)(n)=(\widehat{\varphi}(x) f)(n)+\operatorname{tg}(\pi(n \omega+x)) f(n) .
$$

Тогда для любого $\delta>0$ сушествует такое множество $\Omega_{\delta} \subset[0,1]$ лебеговой меры, большей $1-\delta$, и такое $\varepsilon_{0}=\varepsilon_{0}(\delta, \rho)>0$, что если $\omega \in \Omega_{\delta}$ и $\|\varphi(n, x)\|_{\rho}<\varepsilon_{0}$, то оператор $\widehat{\varphi}_{G M}(x)$ имеет чисто точечный спектр при любом $x$ и его собственные функции экспоненциально убьвают. Это следует, например, из [12].

в3) Оператор Шрёдингера с квазипериодическим потенциалом.

Пусть $X=$ Tor $^{d}=\mathbb{R}^{d} / \mathbb{Z}^{d}-d$-мерньй тор, $x=\left(x_{1}, \ldots, x_{d}\right), 0 \leqslant x_{i} \leqslant 1$ для $1 \leqslant i \leqslant d, \omega=\left(\omega_{1}, \ldots, \omega_{d}\right) \in \mathbb{R}^{d}$ - такой $d$-мерньй вектор, что для любых цельх $n_{1}, n_{2}, \ldots, n_{d+1}$

$$
\sum_{i=1}^{d} n_{i} \omega_{i}+n_{d+1} \neq 0, \quad T^{n} x=\left(x_{1}+n_{1} \omega_{1}, \ldots, x_{d}+n_{d} \omega_{d}\right) \bmod 1
$$

$\mu$ - мера Лебега на торе. Тогда $\left(X,\left\{T^{n}\right\}, \mu\right)$ - эргодическая динамическая система (см. [8]). В одномерном случае, $n \in \mathbb{Z}^{1}$, выбирая произвольный элемент $\varphi(n, x)$ алгебры $A^{\rho}$ при некотором $\rho>0$, мы получаем в качестве $\widehat{\varphi}(x)$ семейство одномерных операторов общего вида с квазипериодическими коэффициентами.

В отличие от примеров а) и б) динамическая система $\left(X,\left\{T^{n}\right\}, \mu\right)$ в данном случае обладает минимальными из возможных статистическими свойствами: она всего лишь эргодична. Это обстоятельство делает невозможным изучение квазипериодических операторов методами, развитыми для исследования операторов с чисто случайными коэффициентами. С другой стороны, простота квазипериодических динамических систем (в противоположность гиперболическим динамическим системам (пример б)) позволяет получить интересные результаты о спектральных свойствах таких семейств операторов.

Дальнейший текст состоит из двух частей, первая из которых содержит обзор некоторых результатов, относящихся к исследованию одномерных разностных операторов Шрёдингера с квазипериодическим потенщиалом и полученных в последние годы; вторая часть содержит доказательство новых результатов о спектре семейств дальнодействующих операторов с квазипериодическими коэффициентами.

В заключение я выражаю глубокую благодарность Я. Г. Синаю за многочисленные стимулируюшие обсуждения вопросов, рассмотренных в этой статье. Я искренне благодарен И. Ю. Арнольд за помош при подготовке рукописи к печати. 


\section{Часть первая.}

\section{Одномерные операторы Шрёдингера с квазипериодическим потенциалом}

Эта часть состоит из двух параграфов и дополнения. Первый параграф посвящен обзору результатов о локализации Андерсона для одномерных разностных операторов Шрёдингера с квазипериодическим потенщиалом общего вида; второй - обзору полученных недавно результатов, относяшихся к одному из самых популярных в этой области примеру: оператору почти-Матье (или оператору Харпера). Дополнение содержит обзор некоторых результатов, относящихся к одномерному непрерывному оператору Шрёдингера с квазипериодическим потенциалом.

\section{§1. Локализация Андерсона для одномерного разностного оператора Шрёдингера с квазипериодическим потенциалом}

В этом параграфе рассматривается семейство операторов

$$
\left(H_{\omega, \varepsilon, x} \psi\right)(n)=\varepsilon[\psi(n+1)+\psi(n-1)]+V(x+n \omega) \psi(n),
$$

действующих в $\ell^{2}\left(\mathbb{Z}^{1}\right)$. Это семейство зависит от трех параметров: иррационального числа $\omega$, действительного числа $\varepsilon$ и циклической переменной $x \in S^{1}$. Мы предполагаем, что $V(x) \in C^{2}\left(S^{1}\right)$. При фиксированных $\omega$ и $\varepsilon$ семейство операторов $H_{\omega, \varepsilon, x}$ определяется функцией $\varphi_{\varepsilon}(n, x), n \in \mathbb{Z}^{1}, x \in S^{1}, \varphi_{\varepsilon}(0, x)=V(x), \varphi_{\varepsilon}(n, x)=\varepsilon$ для $|n|=1, \varphi_{\varepsilon}(n, x)=0$ для $|n|>1$, и ассоциировано с эргодической динамической системой $\left(S^{1},\left\{T_{\omega}^{n}\right\}, \mu\right)$, где $T_{\omega}$ - поворот окружности $S^{1}$ на угол $\omega$, а $\mu$ - мера Лебега на $S^{1}$. Как уже отмечалось во введении, спектр $\sigma\left(H_{\omega, \varepsilon, x}\right)$ оператора $H_{\omega, \varepsilon, x}$ при фиксированных $\omega$ и $\varepsilon$ не зависит от $x$ для почти всех $x$ по мере $\mu$.

Нас интересует более тонкая структура спектра $\sigma\left(H_{\omega, \varepsilon, x}\right)$ : наличие или отсутствие компонент $\sigma_{p p}\left(H_{\omega, \varepsilon, x}\right), \sigma_{a c}\left(H_{\omega, \varepsilon, x}\right), \sigma_{s c}\left(H_{\omega, \varepsilon, x}\right)$ и характер убьвания собственных функций (если таковые имеются). Как мы увидим из дальнейшего, ответ на этот вопрос зависит существенным образом от арифметической природы числа $\omega$ и значения параметра $\varepsilon$.

Мы будем говорить, что $\omega$ удовлетворяет диофантову условию $(D)$ с постоянньм $C>0$ и $\sigma>1$, если

$$
|\{n m\}|>\frac{C}{|n|^{\sigma}}
$$

для всех $n \neq 0$. Здесь фигурные скобки означают дробную часть числа.

Наиболее обший результат о том, когда для семейства операторов $H_{\omega, \varepsilon, x}$ имеет место локализация Андерсона, получен Синаем в [1] (см. также [4]) и Фрелихом, Спенсером и Витвером в [13].

Tеорема 1 (см. [1]). Предположим, что $V(x) \in C^{2}\left(S^{1}\right)$ есть функиия Морса на $S^{1}$ с двумя критическими точками. Тогда для любого $\delta>0$ существуют такое множество $\Omega_{\delta} \subset[0,1]$ лебеговой меры, большей $1-\delta$, и такое $\varepsilon_{0}=\varepsilon_{0}(\delta, V)$, что для почти всех $x$ по мере Лебега при каждом $\omega \in \Omega_{\delta} u|\varepsilon|<\varepsilon_{0}$ оператор $H_{\omega, \varepsilon, x}$ имеет чисто точечныи спектр. Соответствующие собственные функиии убывают әкспоненииально. Спектр $\sigma\left(H_{\omega, \varepsilon, x}\right)$ однократныи и как множество (замыкание множества собственных значений) является канторовым множсеством положительной меры. 
ЗАмЕчАНИЕ. Множество $\Omega_{\delta}$ состоит из таких $\omega$, которые удовлетворяют диофантову условию $(D)$ с фиксированными $\sigma$ и постоянной $C=C(\delta)$, стремящейся к нулю при $\delta \rightarrow 0$.

Опишем основные идеи доказательства теоремы 1 . Мы будем следовать подходу, развитому в [1] и [4].

Доказательство проводится с помошью индуктивной процедуры, на каждом шаге которой строятся приближенные собственные значения (ПСЗ) и соответствуюшие приближенные собственные функции (ПСФ) семейства операторов $H_{\omega, \varepsilon, x}$, которые в пределе дают полный набор собственных значений (С3) и собственных функции (С $\Phi)$ для почти всех $x$.

Случай гладкой функции $V(x)$ принципиально отличается от случая монотонной функции $V(x)$ (см. во введении примеры в1) и в 2)). В последнем случае можно построить СЗ и СФ соответствуюших семейств операторов при помоши индуктивной процедуры типа КАМ-теории, исключив из рассмотрения некоторое подмножество поворотов $\omega$, мера которого стремится к нулю при $\varepsilon \rightarrow 0$. На каждом шаге индуктивной процедуры строятся поправки к С $\Phi$ и С 3 , построенньм на предыдущем шаге, при помощи теории возмущений, по существу, аналогичной обычному ряду теории возмущений для изолированного СЗ конечномерной матрицы.

Для гладкого потенщиала $V(x)$ на каждом шаге индуктивной процедуры ряды обычной теории возмушений содержат бесконечное число малых знаменателей (резонансов) при любом $\omega$. Поэтому индуктивная процедура должна включать в себя построение ПСЗ и ПСФ и в присутствии резонансов.

Из того, что при фиксированных $\omega$ и $\varepsilon$ семейство операторов $H_{\omega, \varepsilon, x}$ ассоциировано с динамической системой $\left(S^{1},\left\{T_{\omega}^{n}\right\}, \mu\right)$, следует, что если функция $\varphi_{\lambda(x)}(n) \in \ell^{2}\left(\mathbb{Z}^{1}\right)$ является СФ оператора $H_{\omega, \varepsilon, x}$ с С $3 \lambda(x)$ :

$$
\left(H_{\omega, \varepsilon, x} \varphi_{\lambda(x)}\right)(n)=\lambda(x) \varphi_{\lambda(x)}(n),
$$

то функция $\widetilde{\varphi}_{\lambda(x)}=\varphi_{\lambda(x)}(n-m)$ является С $\Phi$ оператора $H_{\omega, \varepsilon, T_{\omega}^{m} x}$ c тем же самьм C3 $\lambda(x)$.

Предположим, что экспоненциальная локализация Андерсона имеет место для операторов семейства $H_{\omega, \varepsilon, x}$ при $x$, принадлежаших множеству $S \subset S^{1}$ полной лебеговой меры. Без ограничения обшности, можно считать, что $S T_{\omega}$-инвариантно. В противном случае вместо $S$ можно рассмотреть $\bigcap_{n \in \mathbb{Z}} T_{\omega}^{n} S$.

Предположим также, что имеет место равномерная локализация $\mathrm{C} \Phi$, т.е. для каждой С $\Phi \varphi_{i}(n)$ существуют такие положительные постоянные $A$ и $\gamma$, не зависяшие от $i$, и такое множество $L\left(\varphi_{i}\right) \subset \mathbb{Z}$, являющееся объединением конечного числа конечных интервалов на решетке, что

$$
\left|\varphi_{i}(n)\right|<A e^{-\gamma \operatorname{dist}\left(n, L\left(\varphi_{i}\right)\right)} .
$$

(В ходе доказательства показьвается, что равномерная локализация действительно имеет место.)

Множество $L\left(\varphi_{i}\right)$ будет назьваться сушественным носителем функции $\varphi_{i}(x)$, поскольку, как правило, на $L\left(\varphi_{i}\right) \mathrm{C} \Phi \varphi_{i}$ принимает значения порядка 1 . Конечно, выбор множества $L\left(\varphi_{i}\right)$ и постоянных $A$ и $\gamma$ неоднозначен. Доказательство включает в себя конкретную процедуру однозначного выбора $L\left(\varphi_{i}\right), A$ и $\gamma$. В таком случае имеет смысл говорить о самой левой точке множества $L\left(\varphi_{i}\right)$, которую мы обозначим $\operatorname{pos}\left(\varphi_{i}\right)$. 
Легко убедиться в том, что для того, чтобы найти все С $\Phi \varphi_{i}$ при каждом фиксированном $x \in S^{1}$, достаточно построить С $\Phi \Phi_{i}(x)$, для которых $\operatorname{pos}\left(\Phi_{i}\right)=0$, но для всех $x \in S$. Пусть $\Lambda(x)$ - множество С $3, \Phi(x)$ - множество соответствующих С $\Phi$ с $\operatorname{pos}(\Phi(x))=0$ для $x \in S$. Функции $\Phi_{i}(x)$ со значениями в $\ell^{2}(Z)$ и $\Lambda(x)$ со значениями в $\mathbb{R}$ являются многозначными функциями.

Для построения $\Lambda(x)$ и $\Phi_{i}(x)$ применяется индуктивная процедура, на $s$-м шаге которой для всех $x \in S^{1}$ строятся ПСЗ $\Lambda^{(s)}(x)$ и ПС $\Phi \Phi^{(s)}(x)$, которые также являются многозначными функциями, имеюшими конечное число непрерывных ветвей $\Lambda_{j}^{(s)}(x)$, $\Phi_{j}^{(s)}(x)$, каждая из которых определена на некотором подмножестве окружности $S^{1}$, являющемся либо отрезком, либо объединением конечного числа интервалов. Более детально структура функций $\Lambda_{j}^{(s)}(x)$ и $\Phi_{j}^{(s)}(x)$ будет описана позже.

Функции $\Lambda_{j}^{(s)}(x)$ и $\Phi_{j}^{(s)}(x)$ являются ПСЗ и ПСФ порядка $s$, а именно:

$$
H_{x} \Phi_{j}^{(s)}(x)=\Lambda_{j}^{(s)}(x) \Phi_{j}^{(s)}(x)+\delta_{j}^{(s)}(x),
$$

при этом $\delta_{j}^{(s)}(x)=O\left(\varepsilon^{s}\right),\left\|\delta_{j}^{(s)}\right\|_{\ell^{2}\left(\mathbb{Z}^{1}\right)}<e^{-c s}$, где $c$ - положительная постоянная. (Здесь мы опускаем индексы $\omega$ и $\varepsilon$, имея ввиду, что $\omega$ и $\varepsilon$ фиксированы, $\omega$ удовлетворяет условию $(D), \varepsilon$ достаточно мало.)

Совокупность ПС $\Phi\left\{\varphi_{i}^{(s)}(x)\right\}$ и ПСЗ $\left\{\lambda_{i}^{(s)}(x)\right\}$ для фиксированных $x$ определяется следуюшим образом:

$$
\left\{\lambda_{i}^{(s)}(x)\right\}=\bigcup_{j}\left(\bigcup_{n}^{\prime} \Lambda_{j}^{(s)}(x+n \omega)\right), \quad\left\{\varphi_{i}^{(s)}(x)\right\}=\bigcup_{j}\left(\bigcup_{n}^{\prime} \Phi_{j}^{(s)}(x+n \omega)\right),
$$

где ' означает, что объединение при фиксированном $j$ производится только по таким $n$, для которых $x+n \omega$ принадлежит области определения $\Lambda_{j}^{(s)}(x), \Phi_{j}^{(s)}(x)$.

Доказывается, что совокупность $\left\{\varphi_{i}^{(s)}(x)\right\}$ образует базис в $\ell^{2}\left(\mathbb{Z}^{1}\right)$ (вообще говоря, неортогональньй) при каждом $s$ и $x \in S^{1}$, и что $\left\{\varphi_{i}(x)\right\}=\lim _{s \rightarrow \infty}\left\{\varphi_{i}^{(s)}(x)\right\}$ сушествует при почти всех $x$ и образует ортогональньй базис в $\ell^{2}\left(\mathbb{Z}^{1}\right)$.

Для построения $\Lambda_{j}^{(1)}(x)$ и $\Phi_{j}^{(1)}(x)$ на первом шаге индукции положим $\varepsilon=0$. Тогда $H_{x}$ переходит в оператор умножения на функцию. Легко проверить, что в этом случае $j$ принимает только одно значение, равное $1, \Phi_{1}^{(1)}(x)=\delta_{0 n}, \Lambda_{1}^{(1)}(x)=V(x)$ для любого $x \in S^{1}$. Очевидно, что $\Phi_{1}^{(1)}(x)$ и $\Lambda_{1}^{(1)}(x)$ есть ПСФ и ПСЗ первого порядка.

Опишем подробнее структуру функций $\Lambda_{j}^{(s)}(x), \Phi_{j}^{(s)}(x)$.

1) $\Lambda_{j}^{(s)}(x) \in C^{2}\left(I_{j}^{(s)}\right)$, где $I_{j}^{(s)}$ - область определения ветви $\Lambda_{j}^{(s)}(x)$; каждое свое значение, за исключением критических, функция $\Lambda_{j}^{(s)}(x)$ принимает ровно два раза.

$2)$ Для $s>1$ функция $\Lambda_{j}^{(s)}(x)$ имеет не более одной критической точки, и эта точка невырожденна (если она существует).

$3)$ Для каждой конщевой точки $\bar{x}$ области определения функции $\Lambda_{j}^{(s)}(x)$ найдутся такое $n<$ const $\cdot s$ и такая ветвь $\Lambda_{k}^{(s)}(x)$, что $T_{\omega}^{n} \bar{x}-$ концевая точка области определения $\Lambda_{k}^{(s)}(x)$ и функция $\Lambda_{j k}^{(s)}(x)$, равная $\Lambda_{j}^{(s)}(x)$ в одной полуокрестности точки $\bar{x}$ и равная $\Lambda_{k}^{(s)}\left(T_{\omega}^{-n} x\right)$ в другой полуокрестности точки $\bar{x}$, непрерьвна в $\bar{x}$ вместе с первой и второй производньми. 
4) Диаметр существенного носителя $L\left(\Phi_{j}^{(s)}\right)$ функщии $\Phi_{j}^{(s)}(x)$ не превосходит const ·s при любом $x$.

5) Области значений $\operatorname{Ran}\left(\Lambda_{j}^{(s)}(x)\right)$ и $\operatorname{Ran}\left(\Lambda_{k}^{(s)}(x)\right)$ не пересекаются при $j \neq k$. Для каждой ветви $\Lambda_{j}^{(s)}(x), \Phi_{j}^{(s)}(x)$ указан момент ее появления, т.е. номер шага индукции, на котором она образовалась. Если ветви $\Lambda_{j}^{(s)}(x), \Lambda_{k}^{(s)}(x)$ образовались на $t$-м шаге индукции, $t \leqslant s$, то

$$
\operatorname{dist}\left(\operatorname{Ran}\left(\Lambda_{j}^{(s)}(x)\right), \operatorname{Ran}\left(\Lambda_{k}^{(s)}(x)\right)\right)>C_{1} e^{-C_{2} t}
$$

при некоторых постоянных $C_{1}, C_{2}>0$.

Для того, чтобы перейти от $s$ к $s+1$, построим по $\Lambda_{i}^{(s)}(x)$ и $\Phi_{i}^{(s)}(x)$ ПСЗ $\lambda_{i}^{(s)}(x)$ и ПС $\Phi \varphi_{i}^{(s)}(x)$ для каждого $x \in S^{1}$, как это было уже объяснено.

Мы говорим, что $\lambda_{i}^{(s)}(x)$ является нерезонансным в точке $x$, если для любого $k \neq i$ вьполнено одно из двух условий:

1) $\left|\lambda_{i}^{(s)}(x)-\lambda_{k}^{(s)}(x)\right|>1 / s^{4}$

2) для сушественных носителей $L\left(\varphi_{i}^{(s)}(x)\right)$ и $L\left(\varphi_{k}^{(s)}(x)\right)$ функций $\varphi_{i}^{(s)}(x)$ и $\varphi_{k}^{(s)}(x)$

$$
\operatorname{dist}\left(L\left(\varphi_{i}^{(s)}(x)\right), L\left(\varphi_{k}^{(s)}(x)\right)\right)>\text { const } \cdot \frac{s}{\ln \varepsilon^{-1}},
$$

$$
\text { где } L\left(\varphi_{i}^{(s)}(x)\right)=T_{\omega}^{-n} L\left(\Phi_{j}^{(s)}(x)\right) \text {, если } \varphi_{i}^{(s)}(x)=\Phi_{j}^{(s)}(x+n \omega) \text {. }
$$

В этом случае ПСЗ $\lambda_{i}^{(s+1)}(x)$ и ПСФ $\varphi^{(s+1)}(x)$ определяются с помощью ряда теории возмушений, аналогичного ряду теории возмушений для изолированного собственного значения конечномерной матрицы. Причем в этом ряде достаточно сохранить только члены первого порядка. А именно, мы полагаем:

$$
\begin{aligned}
& \varphi_{i}^{(s+1)}(x)=\varphi_{i}^{(s)}(x)+\sum_{j \neq i}^{\prime} \frac{\left(\delta_{i}^{(s)}(x), \varphi_{j}^{(s)}(x)\right)}{\lambda_{i}^{(s)}(x)-\lambda_{j}^{(s)}(x)} \varphi_{j}^{(s)}(x), \\
& \lambda_{i}^{(s+1)}(x)=\lambda_{i}^{(s)}+\left(\delta_{i}^{(s)}(x), \varphi_{i}^{(s)}(x)\right) .
\end{aligned}
$$

Здесь ' означает, что суммирование проводится только по таким $j$, для которых условие 2) не вьполнено. Включение в сумму дополнительных членов дало бы только поправку более высокого порядка, чем $O\left(\varepsilon^{s}\right)$. Это следует из экспоненциального убывания ПСФ вне их сушественных носителей.

Мы полагаем $L\left(\varphi_{i}^{(s+1)}\right)=L\left(\varphi_{i}^{(s)}\right)$ и определяем ветви ПСЗ $\Lambda_{j(i)}^{(s+1)}$ и ПС $\Phi \Phi_{j(i)}^{(s+1)}$ следуюшим образом:

$$
\Lambda_{j(i)}^{(s+1)}(x+n \omega)=\lambda_{i}^{(s+1)}(x), \quad \Phi_{j(i)}^{(s+1)}(x+n \omega)=\varphi_{i}^{(s+1)}(x) .
$$

Тем самым мы определили ветви ПСЗ $\Lambda_{j(i)}^{(s+1)}(x)$ и ПСФ $\Phi_{j(i)}^{(s+1)}(x)(s+1)$-го порядка для всех значений $x$, для которых $\lambda_{i}^{(s)}(x)$ при отображении $\left\{\Lambda_{i}^{(s)}\right\} \rightarrow\left\{\lambda_{i}^{(s)}\right\}$ 
нерезонансно в точке $x$. Из оценок ряда теории возмущений вытекает, что новые ветви $\Lambda_{i}^{(s+1)}(x)$ для нерезонансных $x$ отличаются от $\Lambda_{i}^{(s)}(x)$ на величину, не превосходящую $O\left(\varepsilon^{s}\right)$, а для соответствующих $\Phi_{i}^{(s+1)}(x)$ и $\Phi_{i}^{(s)}(x)$

$$
\left\|\Phi_{i}^{(s+1)}(x)-\Phi_{i}^{(s)}(x)\right\|_{\ell^{2}\left(\mathbb{Z}^{1}\right)}<\exp (-c s) .
$$

Если для $\lambda_{i}^{(s)}(x)$ не вьполнено ни одно из условий 1$)$ и 2$), \lambda_{i}^{(s)}(x)$ назьвается резонансным в точке $x$. В этом случае сушествует такое $k \neq i$, что

$$
\begin{aligned}
\left|\lambda_{i}^{(s)}(x)-\lambda_{k}^{(s)}(x)\right| & \leqslant \frac{1}{s^{4}} \\
\operatorname{dist}\left(L\left(\varphi_{i}^{(s)}(x)\right), L\left(\varphi_{k}^{(s)}(x)\right)\right) & <\operatorname{const} \frac{s}{\ln \varepsilon^{-1}} .
\end{aligned}
$$

Тогда пара $\lambda_{i}^{(s)}(x), \lambda_{k}^{(s)}(x)$ называется резонансной парой порядка $s$.

Из того, что многозначная функция $\Lambda^{(s)}(x)$ принимает каждое свое значение не более чем в двух точках, и из того, что $\omega$ удовлетворяет диофантову условию $(D)$, следует, что для данного $i$ существует не более одной резонансной пары $\lambda_{i}^{(s)}(x), \lambda_{k}^{(s)}(x)$ $s$-ого порядка в точке $x$.

Из свойств функций $\Lambda_{i}^{(s)}(x)$ следует, что функции $\lambda_{j}^{(s)}(x)$ являются гладкими (класса $C^{2}$ ) функциями в некоторой окрестности точки $x$. Следовательно, компонента множества точек $y$, содержащих точку $x$ и удовлетворяющих неравенству

$$
\left|\lambda_{i}^{(s)}(y)-\lambda_{k}^{(s)}(y)\right| \leqslant \frac{1}{s^{4}}
$$

является отрезком. Из оценок первых двух производных функщий $\lambda_{i}^{(s)}(y), \lambda_{k}^{(s)}(y)$ сверху и снизу, получаемых на каждом шаге индукщии, вытекает, что длина этого отрезка не меньше const $\cdot s^{-5}$ и не превосходит const $\cdot s^{-2}$.

Этот отрезок назьвается резонансной зоной (РЗ) порядка $s$.

Для такой $\mathrm{P} 3 \lambda_{i}^{(s+1)}(x)$ и $\lambda_{k}^{(s+1)}(x)$ и соответствующие $\varphi_{i}^{(s+1)}(x)$ и $\varphi_{k}^{(s+1)}(x)$ определяются следуюшим образом. Сначала определяются промежуточные ПСЗ $\widetilde{\lambda}_{i}^{(s+1)}(x), \widetilde{\lambda}_{k}^{(s+1)}(x)$ и ПСФ $\widetilde{\varphi}_{i}^{(s+1)}(x), \widetilde{\varphi}_{k}^{(s+1)}(x)$ при помощи ряда теории возмушений, вьписанного вьше. При этом из суммы дополнительно исключается слагаемое $j=k$ для $\widetilde{\varphi}_{i}^{(s+1)}(x)$ и $j=i$ для $\widetilde{\varphi}_{k}^{(s+1)}(x)$. Затем $\widetilde{\lambda}_{i}^{(s+1)}(x)$ и $\widetilde{\lambda}_{k}^{(s+1)}(x)$ строятся как линейные комбинации функций $\widetilde{\varphi}_{i}^{(s+1)}(x)$ и $\widetilde{\varphi}_{k}^{(s+1)}(x)$ :

$$
\begin{aligned}
& \varphi_{i}^{(s+1)}(x)=A_{+} \widetilde{\varphi}_{i}^{(s+1)}(x)+B_{+} \widetilde{\varphi}_{k}^{(s+1)}(x), \\
& \varphi_{k}^{(s+1)}(x)=A_{-} \widetilde{\varphi}_{i}^{(s+1)}(x)+B_{-} \widetilde{\varphi}_{k}^{(s+1)}(x) .
\end{aligned}
$$

Коэффициенты $A_{ \pm}, B_{ \pm}$совпадают с элементами ортогональной матрицы, приводящей матрищу

$$
Q_{i, k}(x)=\left(\begin{array}{cc}
\tilde{\lambda}_{i}^{(s+1)}(x) & \left(\widetilde{\varphi}_{i}^{(s+1)}(x), \widetilde{\varphi}_{k}^{(s+1)}(x)\right) \\
\left(\widetilde{\varphi}_{i}^{(s+1)}(x), \widetilde{\varphi}_{i}^{(s+1)}(x)\right) & \widetilde{\lambda}_{k}^{(s+1)}(x)
\end{array}\right)
$$


к диагональному виду. Новые ПСЗ $\lambda_{i}^{(s+1)}(x)$ и $\lambda_{k}^{(s+1)}(x)$ определяются как СЗ матрицы $Q_{i, k}(x)$. Мы полагаем $L\left(\varphi_{i}^{(s+1)}\right)=L\left(\varphi_{k}^{(s+1)}\right)=L\left(\varphi_{i}^{(s)}\right) \cup L\left(\varphi_{k}^{(s)}\right)$.

Пусть $\operatorname{pos}\left(L\left(\varphi_{i}^{(s)}(x)\right)\right)=n$. Тогда для точки $y=x+n \omega$, где $x$ принадлежит РЗ, определяются две новые ветви ПС $\Phi \Phi_{i^{\prime}}^{(s+1)}(y), \Phi_{i^{\prime \prime}}^{(s+1)}(y)$ и ПСЗ $\Lambda_{i^{\prime}}^{(s+1)}(y), \Lambda_{i^{\prime \prime}}^{(s+1)}(y)$ :

$$
\begin{array}{ll}
\Lambda_{i^{\prime}}^{(s+1)}(y)=\lambda_{i}^{(s+1)}(x), & \Lambda_{i^{\prime \prime}}^{(s+1)}(y)=\lambda_{k}^{(s+1)}(x), \\
\Phi_{i^{\prime}}^{(s+1)}(y)=\varphi_{i}^{(s+1)}(x), & \Phi_{i^{\prime \prime}}^{(s+1)}(y)=\varphi_{j}^{(s+1)}(x),
\end{array}
$$

где нумерация ветвей определяется некоторьм стандартньм образом, так что различные ветви имеют различные номера. Кроме того, доказывается, что число ветвей $\Lambda_{i}^{(s)}(x), \Phi_{i}^{(s)}(x)$ не превосходит const $\cdot s$.

О ветвях ПСЗ $\Lambda_{i^{\prime}}^{(s)}(x), \Lambda_{i^{\prime \prime}}^{(s)}(x)$ и ПС $\Phi \Phi_{i^{\prime}}^{(s)}(x), \Phi_{i^{\prime \prime}}^{(s)}(x)$ говорят, что они образовались на шаге $s$.

Образование двух новых ветвей ПСЗ и ПСФ внутри РЗ называется расщеплением резонанса.

Полученные новые ветви ПСЗ и ПС $\Phi(s+1)$-го порядка, вообще говоря, не являются малыми возмушениями ПСЗ и ПС $\Phi$-го порядка. Важно отметить, что из оценок ПС $\Phi$ и производных ПСЗ по $x$, получаемых в ходе индуктивного процесса, доказывается оценка снизу для расщепления резонанса:

$$
\left|\lambda_{i}^{(s+1)}(x)-\lambda_{k}^{(s+1)}(x)\right|>C_{1} \varepsilon^{-C_{2} s},
$$

где $C_{1}, C_{2}$ - положительные постоянные.

Получение этой оценки является технически самой трудной частью доказательства. При этом используется конкретньй вид оператора Шрёдингера и специальное представление погрешности $\delta_{i}^{(s)}$.

Благодаря этой оценке расщепления мы получаем следующее важное свойство: между множествами значений образовавшихся при расщеплении резонансановых ветвей образуется щель. Эта щель может лишь незначительно сузиться при последуюших шагах индукции. Из оценки размеров РЗ $s$-го порядка и диофантовости числа $\omega$ следует, что расстояние между последовательными моментами $s_{1}$ и $s_{2}$ первого попадания точки $x$ в $\mathrm{P} 3$ превосходит $C_{1}^{\prime} e^{C_{2} s_{1}} \ln \varepsilon^{-1}$, (разреженность резонансов) и что суммарная мера всех РЗ конечна. Поэтому почти каждая точка $x \in S^{1}$ принадлежит какой-либо РЗ только конечное число раз. Это позволяет доказать сходимость $\lambda_{i}^{(s)}(x)$ и $\varphi_{i}^{(s)}(x)$ при $s \rightarrow \infty$ для почти всех $x$. Полнота предельных собственных функций вытекает из полноты допредельных ПС $\Phi \varphi_{i}^{(s)}(x)$ при каждом $s$.

Из оценок размера щелей и всюду плотности траектории $\left\{T_{\omega}^{n} x\right\}$ любой точки $x$ легко показать, что замькание множества значений функции $\Lambda(x)=\lim _{s \rightarrow \infty} \Lambda^{(s)}(x)$ есть канторовское множество положительной меры, которое и является спектром $H_{\omega, \varepsilon, x}$.

Можно сказать, что теорема 1 устанавливает метрическую устойчивость экспоненциальной локализации Андерсона: чисто точечньй спектр и экспоненщиальное убьвание С $\Phi$ имеют место для множества полной меры значений $x \in S$.

$\mathrm{C}$ другой стороны, существование чисто точечного спектра является крайне неустойчивым свойством эргодического семейства операторов с топологической точки зрения. 
Пусть $V(n)$ - почти периодическая функция на $\mathbb{Z}^{1}$. Напомним, что $V(n)$ назьвается почти периодической на $\mathbb{Z}^{1}$, если $V(n)$ ограниченна и множество $\left\{V_{m}(n)=V(n-m)\right\}$ имеет компактное замыкание в $\ell^{\infty}\left(\mathbb{Z}^{1}\right)$. Это замыкание $\Omega_{V}$ назьвается оболочкой почти периодической функции $V(n)$.

В частности, если $v(x)$ - непрерьвная функция на $S^{1}$, то последовательность $V_{x_{0}}(n)=v\left(x_{0}+n \omega\right)$ при фиксированном $x_{0} \in S^{1}$ и иррациональньм $\omega$ определяет почти периодическую функцию $V_{x_{0}}(n)$ на $\mathbb{Z}^{1}$. Легко проверить, что оболочка $\Omega_{V_{x_{0}}}$ функции $V_{x_{0}}(n)$ состоит из всех функций $V_{x}(n)=v(x+n \omega), x \in S^{1}$.

В работе С. Житомирской и Б. Саймона [14] доказаны следующие утверждения.

Теорема 2. Пусть $V(n)$ - четная почти периодическая функиия, т.е. $V(n)=$ $V(-n)$. Тогда существует плотное $G_{\delta}$-множсество $U \subset \Omega_{V}$ такое, что если $u(n) \in U$, mo оператор $\left(H_{U} \Psi\right)(n)=\Psi(n+1)+\Psi(n-1)+u(n) \Psi(n)$ как оператор в $\ell^{2}(\mathbb{Z})$ не имеет собственных значений.

ТЕОРема 3. Пусть выполнены предположения теоремы 2. Предположим дополнительно, что существует хотя бы одно $u(n) \in \Omega_{V}$, для которого оператор $H_{u}$ не имеет абсолютно непрерывного спектра. Тогда для плотного $G_{\delta}$-подмножества $\widetilde{U} \subset \Omega_{V}$ оператор $H_{\widetilde{u}}(\widetilde{u} \in \widetilde{U})$ имеет чисто сингулярно непрерывный спектр.

В частности, взяв в качестве $V(x)$ в теореме 1 четную функцию, мы получим, что для всюду плотного $G_{\delta}$-подмножества окружности $S^{1}$ оператор $H_{\omega, \varepsilon, x}$ имеет чисто сингулярно непрерьвньй спектр.

Идея доказательства теорем 2 и 3 применительно к семейству операторов $H_{\omega, \varepsilon, x}$ с четным потенциалом $V(x)$ состоит в том, что соответствующее множество $\widetilde{U}$ строится как множество таких функций $V_{x}(n)$, для которых $x$ принадлежит бесконечному числу РЗ.

Теорема 1 относится к потенциалу с одной частотой $\omega$. Случай двух частот рассмотрен Я.Г. Синаем и В.А. Чулаевским в [2] и [4].

Предположим, что действительная функция $V(x) \in C^{2}\left(\mathrm{Tor}^{2}\right)$ удовлетворяет следуюшим условиям:

I. $V(x)$ имеет конечное число критических точек $x^{1}, \ldots, x^{n}$, и все эти критические точки невырожденны. Значения функции $V(x)$ в различных критических точках различны.

II. Множества уровней $A(V, c)=\left\{x \in\right.$ Tor $\left.^{2}: V(x)=c\right\}$ состоят из конечного числа замкнутых кривых, которые могут иметь самопересечения только в критических седловых точках.

III. Для любого $\omega \in[0,1] \times[0,1]$ множество $\left\{x \in\right.$ Tor $\left.^{2}: V(x+\omega)-V(x-\omega)=0\right\}$ состоит из конечного числа замкнутых кривых, каждая из которых имеет конечное число самопересечений. Ограничение функции $V$ на любую из этих кривых имеет конечное число критических точек, которые могут быть вырожденными только для векторов $\omega$, принадлежаших объединению конечного числа замкнутых кривых или дуг.

Рассмотрим семейство операторов

$$
\left(H_{\omega, \varepsilon, x} \Psi\right)(n)=(\Psi(n+1)+\Psi(n-1))+V(x+n \omega) \Psi(n),
$$

где $n \in \mathbb{Z}^{1}$ 
В [2] и [4] доказана следуюшая

ТеОРема 4. Пусть для $V(x)$ выполнены условия I-III. Тогда для любого $\delta>0$ существуют такое множество $\Omega_{\delta} \subset[0,1] \times[0,1]$ лебеговой мерь, большей $1-\delta$, $u$ такая постоянная $\varepsilon_{0}=\varepsilon_{0}(\delta, V)>0$, что для любого $\varepsilon,|\varepsilon|<\varepsilon_{0}$, и любого $\omega \in \Omega_{\delta}$ оператор $H_{\omega, \varepsilon, x}$ для почти всех $x$ имеет чисто точечный спектр, $и$ соответствующие собственные функции әкспоненциально убывают.

Доказательство теоремы 4 аналогично доказательству теоремы 1 , но технически более сложно, так как типичная кратность резонанса в этом случае может равняться 2 или 3 , а не только 2, как в случае одной частоты.

Основное отличие двухчастотного случая от одночастотного заключается в том, что в случае двух частот $\sigma\left(H_{\omega, \varepsilon, x}\right)$ не содержит шелей.

\section{$\S 2$. О спектре оператора почти-Матье}

Почти-Матье оператор - это разностньй оператор Шрёдингера с квазипериодическим потенциалом, порожденным функцией $\lambda \cos 2 \pi x$ :

$$
H_{\omega, \lambda, x}^{M} \Psi(n)=\Psi(n+1)+\Psi(n-1)+\lambda \cos (2 \pi(x+n \omega)) \Psi(n)
$$

где $\lambda$ - действительный параметр. Из соображений симметрии следует, что достаточно рассмотреть случай $\lambda>0$.

Этот оператор возникает во многих задачах теоретической физики и в физической литературе иногда называется оператором Харпера.

Почти-Матье оператор является одним из самых популярных примеров и ему посвящено огромное число математических и физических работ. Этот пример демонстрирует удивительное многообразие спектральных свойств в зависимости от значений параметров $\omega, \lambda$ и $x$.

В этом параграфе будут приведены формулировки лишь нескольких важных результатов, полученных в самое последнее время и связанных с наличием или отсутствием точечной компоненты спектра оператора (1). Мы с самого начала исключаем из рассмотрения рациональные $\omega$, хотя уже в этом случае для критического значения $\lambda=2$ появляются интересные и до сих пор до конца нерешенные задачи (бабочка Хофштадера).

Прежде всего, отметим, что спектр $\sigma(\omega, \lambda, x)$ зависит от арифметической природы иррационального $\omega$. Как было описано в $\S 1$, для диофантовых $\omega$ и достаточно больших $\lambda\left(\lambda>\lambda_{0}(\omega)\right)$ спектр оператора $H_{\omega, \lambda, x}^{M}$ - канторово множество положительной меры. С другой стороны, в работе Аврона и Саймона [15] было доказано, что лебегова мера $\left|\sigma\left(H_{\omega, \varepsilon, x}\right)\right|$ спектра $\sigma(\omega, \lambda, x)$ оператора $H_{\omega, \lambda, x}^{M}$ не зависит от $x$ при фиксированных $\omega$ и $\lambda$ ( $\omega$ иррационально). Следовательно, при диофантовых $\omega$ и $\lambda>\lambda_{0}(\omega)$ при всех $x$ спектр $\sigma(\omega, \lambda, x)$ имеет положительную меру.

Согласно теореме Эрмана [16] показатель Ляпунова $\gamma(E) \geqslant \ln \frac{|\lambda|}{2}$ при всех $E$. Из теоремы Ишии-Пастура-Котани (см. [6]) следует, что при $|\lambda|>2$ спектр $\sigma(\omega, \lambda, x)$ не содержит абсолютно непрерывной компоненты. 
Основная гипотеза относительно природы спектра $\sigma(\omega, \lambda, x)$ состоит в следующем: для $\lambda>2$ при типичных $\omega$ и для почти всех $x$ спектр оператора $H_{\omega, \lambda, x}^{M}$ чисто точечный;

для $\lambda<2$ при типичных $\omega$ и для почти всех $x$ спектр оператора $H_{\omega, \lambda, x}^{M}$ абсолютно непрерывный. ${ }^{1}$

$\mathrm{K}$ настояшему времени эта гипотеза не доказана в полной мере. Последние результаты, поддерживаюшие эту гипотезу, принадлежат С. Житомирской для $\lambda>2$ и Й. Ласту для $\lambda<2$.

Рассмотрим множество $\Omega=\left\{x \in S^{1}\right.$ и для каждого $s>1$ неравенство $\left|\sin 2 \pi\left(x+\frac{k}{2} \omega\right)\right|<k^{-s}$ вьполняется для бесконечного числа целых положительных $k\}$. Легко видеть, что лебегова мера множества $\Omega$ равна нулю.

Предположим также, что $\omega$ диофантово.

Кроме того, введем еше одно условие на типичность $\omega$ : сушествует такое $r>1$, что

$$
\frac{C}{q_{n}^{r}}<\left|p_{n}-\omega q_{n}\right|=\frac{O(1)}{q_{n}}
$$

при $n \rightarrow \infty$ при некоторой постоянной $C>0$. Здесь $\frac{p_{n}}{q_{n}}-n$-я подходящая дробь разложения числа $\omega$ в непрерьвную дробь.

Tеорема 1 (см. [17]). 1. Пусть $\lambda \geqslant 15$ и $H_{\omega, \lambda, x}^{M}$ имеет место локализачия Андерсона для почти всех $x$, т.е. при почти каждом $x$ имеется полный набор әкспоненциально убывающих собственных функиий.

2. Положим $\widetilde{\sigma}=\{E \in \sigma(\omega, \lambda, x)$ и для любого $\varepsilon>0|[E-\varepsilon, E+\varepsilon] \cap \sigma(\omega, \lambda, x)|>0\}$. Предположим, что ш удовлетворяет условию $\left(D_{1}\right)$. Тогда для любого $2<\lambda<15$

a) $\left|\sigma_{p p}(\omega, \lambda, x)\right|=|\sigma(\omega, \lambda, x)|$;

б) $\sigma_{s c}(\omega, \lambda, x)$ (если такая компонента существует) имеет меру нуль и нигде неплотно в $\widetilde{\sigma}(\omega, \lambda, x)$ в относительной топологии;

в) если $x \notin \Omega$, то множество собственных значений оператора $H_{\omega, \lambda, x}^{M}$ плотно в $\widetilde{\sigma}$ и соответствующие собственнье функции әкспоненциально убиввют.

ЗАмЕчАНИЕ. В работе [18] показано, что

$$
|\sigma(\omega, \lambda, x)|>2 \lambda-4 \text {. }
$$

Еще раз подчеркнем, что природа спектра $\sigma(\omega, \lambda, x)$ сушественно зависит от арифметической природы числа $\omega$.

В частности, предположим, что $\omega$ является числом Лиувилля, т.е. для любого целого положительного $k$ сушествуют такие $p_{k}, q_{k}$, что

$$
\left|\omega-\frac{p_{k}}{q_{k}}\right|<K^{-q_{k}} .
$$

Аврон и Саймон в [19] доказали следующее утверждение.

\footnotetext{
${ }^{1}$ Другая гипотеза относится к критическому случаю $\lambda=2$ : для типичных $\omega$ и почти всех $x$ оператор $H_{\omega, \lambda, x}^{M}$ имеет сингулярно непрерывньй спектр. По поводу ее доказательства см. [29] (прим. при корректуре).
} 
ТЕОРема 2. Если $\omega-$ число Лиувилля $и \lambda>2$, оператор $H_{\omega, \lambda, x}^{M}$ имеет чисто сингулярно непрерывный спектр для почти всех $x$.

Перейдем теперь к случаю $\lambda<2$. Исследование спектра в этом случае основано на одном общем утверждении, устанавливающем связь между спектром эргодического семейства разностных операторов с потенциалом $V(x)$ и спектром его периодических аппроксимаций. Пусть

$$
\left(H_{V}(x) \psi\right)(n)=\psi(n+1)+\psi(n-1)+V\left(T^{n} x\right) \psi(n)
$$

есть семейство операторов Шрёдингера, определяемое ограниченной функцией $V(x)$ и ассоциированное с эргодической динамической системой $\left(X,\left\{T^{n}\right\}, \mu\right), n \in \mathbb{Z}^{1}$. Как уже отмечалось в $\S 1$, множество

$$
A=\{E: \gamma(E)=0\}
$$

не зависит от $x$ для почти всех $x$, где $\gamma(E)$ - показатель Ляпунова. Теорема ИшииПастура-Котани [6] устанавливает следующую связь между абсолютно непрерьвной компонентой $\sigma_{a c}\left(H_{V}(x)\right)$ спектра оператора $H_{V}(x)$ и множеством $A$ :

$$
\sigma_{a c}\left(H_{V}(x)\right)=\bar{A}^{e s s},
$$

где $A^{e s s}=\{E \in \mathbb{R}:|A \cap[E-\varepsilon, E+\varepsilon]|>0$ для любого $\varepsilon>0\}$. Следовательно, $A$ содержится в $\sigma_{a c}\left(H_{V}(x)\right)$ с точностью до множества меры 0 и $\left|\sigma_{a c}\left(H_{V}(x)\right)\right| \geqslant|A|$.

Для каждого $x$ и целого положительного $m$ определим периодический потенциал $V_{m}^{x}(n)$, полагая

$$
\begin{gathered}
V_{m}^{x}(n)=V\left(T^{n} x\right) \text { для } n=1,2, \ldots m, \\
V_{m}^{x}(n+k m)=V_{m}^{x}(n) \text { для любого } k \in \mathbb{Z}^{1} .
\end{gathered}
$$

Рассмотрим семейство операторов Шрёдингера с периодическим потенщиалом $V_{m}^{x}$ :

$$
\left(H_{m}^{x} \psi\right)(n)=\psi(n+1)+\psi(n-1)+V_{m}^{x}(n) \psi(n) .
$$

Обозначим его спектр $\sigma_{m}^{x}$.

TeOpema 3 (см. [20]). Для почти всех $x$ nо мере $\mu$

$$
\left|\limsup _{m \rightarrow \infty} \sigma_{m}^{x} \backslash A\right|=0
$$

где, как обично

$$
\limsup _{m \rightarrow \infty} \sigma_{m}^{x}=\bigcap_{l=1}^{\infty} \bigcup_{m=l}^{\infty} \sigma_{m}^{x} .
$$

Из этой теоремы непосредственно вытекает, что для почти всех $x$

$$
\limsup _{m \rightarrow \infty}\left|\sigma_{m}^{x}\right| \leqslant|A| \leqslant\left|\sigma_{a c}\left(H_{V}(x)\right)\right|
$$

Вернемся к оператору почти-Матье $H_{\omega, \lambda, x}^{M}$. В [20] для этого оператора доказана следующая 
ТЕОремА 4. 1) Для любого иррачионального $\omega$ и почти всех $x$

$$
\left|\sigma_{a c}(\omega, \lambda, x)\right| \geqslant|A(\omega, \lambda, x)| \geqslant 4-\left(2+\frac{\pi}{\sqrt{5}}\right) \lambda .
$$

2) Если $0 \leqslant \lambda<2$ и существует такая последовательность рациональных чисел $\left\{p_{k} / q_{k}\right\}_{k=1}^{\infty}$, что

$$
\lim _{k \rightarrow \infty} q_{k}^{2}\left|\omega-\frac{p_{k}}{q_{k}}\right|=0
$$

mo

$$
|A(\omega, \lambda, x)|=\left|\sigma_{a c}(\omega, \lambda, x)\right|=|\sigma(\omega, \lambda, x)|=4-2 \lambda
$$

для почти всех $x$.

Сделаем несколько замечаний по поводу теоремы 4.

1. Условие $\left(D_{2}\right)$ вьполняется для почти всех $\omega$.

2. Гипотеза о том, что $|\sigma(\omega, \lambda, x)|=4-2 \lambda$ для всех иррациональных $\omega$ и $0 \leqslant \lambda<2$, принадлежит Андре и Обри [21]. Она исследовалась Таулесом в [22] для рациональных $\omega$. В работе Аврона, Ван Муша и Саймона [23] была доказана оценка снизу: $|\sigma(\omega, \lambda, x)| \geqslant 4-2 \lambda$.

\section{Дополнение к части первой.}

\section{О непрерывном одномерном уравнении Шрёдингера с квазипериодическим потенциалом}

Это дополнение содержит формулировки результатов Л. Элиасона [24], дающих, по существу, окончательное решение вопроса о структуре спектра непрерывного одномерного оператора Шрёдингера с малым квазипериодическим потенциалом.

В статье [24] изучаются решения уравнения Шрёдингера

$$
(L y)(t)=-y^{\prime \prime}(t)+q_{\omega}(t) y(t)=E y(t)
$$

для квазипериодического потенциала $q_{\omega}(t)$ с частотным вектором $\omega$ и для больших энергий $E$ или для малых потенциалов $q$.

Иными словами, функция $q_{\omega}(t)$ может быть описана следующим образом. Пусть $\omega=\left(\omega_{1}, \ldots, \omega_{d}\right) \in \mathbb{R}^{d}$ и $q(x)$ - функция на $d$-мерном торе Tor ${ }^{d}=\mathbb{R}^{d} / \mathbb{Z}^{d}$. Рассмотрим поток $S^{t}$ на торе $\operatorname{Tor}^{d}: S^{t} x^{0}=\left(x_{1}^{0}+\omega_{1} t, \ldots, x_{d}^{0}+\omega_{d} t\right)$, где $x^{0}=\left(x_{1}^{0}, \ldots, x_{d}^{0}\right)$ - произвольная точка на $\operatorname{Tor}^{d}$. Тогда $q_{\omega}(t)=q\left(S^{t} x^{0}\right)$. Функция $q_{\omega}(t)$ зависит от выбора начальной точки $x^{0}$, но мы опускаем указание на эту зависимость, так как утверждения справедливы для любой $x^{0}$.

Мы предполагаем, что $q: \operatorname{Tor}^{d} \rightarrow \mathbb{R}$ аналитична в некоторой комплексной окрестности $|\operatorname{Im} x|<r$. Положим

$$
|q|_{r}=\sup _{|\operatorname{Im} x|<r}|q(x)| .
$$

Эта норма аналогична $\|\cdot\|_{\rho}$, определенной ранее во введении. Конечность $|q|_{r}$ означает экспоненциальную скорость убывания коэффициентов Фурье

$$
a_{n}=\frac{1}{(2 \pi)^{d}} \int_{\text {Tor }^{d}} e^{2 \pi i(n, x)} q(x) d x:\left|a_{n}\right|<\text { const } \cdot e^{-r^{\prime}|n|}
$$

для любого $r^{\prime}<r$. Здесь $(n, x)$ - скалярное произведение векторов $n \in \mathbb{Z}^{d}$ и $x=\left(x_{1}, \ldots, x_{d}\right) \in \operatorname{Tor}^{d}, d x=d x_{1} d x_{2} \ldots d x_{d}$. 
В работе [24] изучается вопрос о сушествовании решений уравнения (1) типа решений Флоке (или волн Блоха), т.е. решений вида $y(t)=e^{i k t}\left(p_{1}(t)+t p_{2}(t)\right)$ с постоянным $k$ и квазипериодическими функциями $p_{1}(t), p_{2}(t)$, имеющими вектор частот $\omega$ или $\omega / 2$. Одновременно изучается спектр $\sigma(\bar{L})$ самосопряженного оператора $\bar{L}$, являющегося расширением оператора $L$ с областью определения $C_{0}^{\infty}(\mathbb{R})$ и действующего в $L^{2}(\mathbb{R})$.

Предполагается, что вектор $\omega$ диофантов, т.е.

$$
|(n, \omega)| \geqslant|n|^{-\tau}, \quad n \in \mathbb{Z}^{d} \backslash 0,
$$

$\mathrm{c} \tau>d-1$.

Для того, чтобы сформулировать результаты, необходимо определить понятие числа врашения для уравнения (1).

Пусть $A: R \rightarrow \mathrm{GL}(2, \mathbb{R}), A(0)=I$, есть непрерьвное отображение и $V \in \mathbb{R}^{2} \backslash 0$. Положим

$$
\Phi(t, V)=\arg (A(t) V),
$$

$\Phi(t, V)$ есть многозначная непрерывная функция на комплексной плоскости. Выбирая

$$
0 \leqslant \arg V<2 \pi
$$

мы превратим $\Phi(t, V)$ в однозначную функцию, непрерьвную в $\mathbb{R} \times \mathbb{R}^{2} \backslash([0, \infty] \times 0)$.

Если предел

$$
\lim _{t \rightarrow \infty} \frac{\Phi(t, V)}{t}
$$

существует, то он не зависит от $V$ и назьвается числом врашения отображения $A$. Мы обозначим этот предел $\rho_{A}$.

Запишем уравнение (1) в векторной форме:

$$
Y^{\prime}(t)=\left(\begin{array}{cc}
0 & 1 \\
q_{\omega}(t)-E & 0
\end{array}\right) Y(t)
$$

где $Y(t)=\left(y^{\prime}(t), y(t)\right)$ есть двумерный вектор. Тогда если $Y(t)$ - решение этой системы уравнений с начальньми данными $Y_{0}(0)$, то

$$
Y(t)=A_{E}(t) Y_{0}(0)
$$

$A_{E}(t) \in \mathrm{GL}(2, \mathbb{R})$ - непрерывное отображение $\mathbb{R} \rightarrow \mathrm{GL}(2, R)$ и $A(0)=I$. В [25] доказано, что если частоты $\omega_{1}, \ldots, \omega_{d}$ рационально независимы, то отображение $A_{E}(t)$ имеет число врашения $\rho(E)$, которое назьвается числом врашения уравнения $(1) . \rho(E)$ - монотонная и непрерывная функция.

Пусть $M=\left\{\frac{(n, \omega)}{2}, n \in \mathbb{Z}^{d}\right\}$ - модуль, порожденньй получастотами функции $q_{\omega}$. В [25] показано, что

$$
\sigma(\bar{L})=\overline{R \backslash \rho^{-1}(M)} .
$$

Связные компоненты множества $\operatorname{Int}\left(\rho^{-1}(M)\right)$ образуют щели в спектре оператора $\bar{L}$. Щель называется коллапсируюшей, если она состоит из одной точки $E$ такой, что $\rho(E) \in M$ и $\rho^{-1}(\rho(E))=E$. 
Назовем число $\rho$ диофантовым, если

$$
\left|\rho-\frac{(n, \omega)}{2}\right|>K^{-1}|n|^{-\sigma}, \quad n \in \mathbb{Z}^{d},
$$

для некоторых положительных $K$ и $\sigma$, и рациональным (относительно $M$ ), если $\rho \in M$.

Теперь мы в состоянии сформулировать первьй результат из [24]. Положим $E_{c}(s)=(s / c)^{2}$ для $s \geqslant C$ и $E_{c}(s)=-\infty$ для $s<C$, где $C>0$ - постоянная.

Tеорема 1. Существует такое $C_{0}=C_{0}(\tau, r)$, что для $E>E_{C_{0}}\left(|q|_{r}\right)$ сnраведливо одно из двух утвержсдений:

I) если $\rho(E)$ диофантово или рационально, то существуют матрица $Q(E) \in$ $\mathrm{sl}(2, \mathbb{R})$, зависящая от $E$, для которой

$$
Y(t)=Z_{\omega / 2}(t) e^{Q t}
$$

II) если $\rho($ Е) не является ни диофантовым, ни рациональнылм, то

$$
\lim _{|t| \rightarrow \infty}|Y(t)-Y(0)|<\frac{1}{2} Y(0) \quad u \quad \lim _{|t| \rightarrow \infty} \frac{Y(t)}{t}=0
$$

ЗАмЕчАниЕ. Теорема 1 есть утверждение о приводимости уравнения (2), так как

$$
\frac{d}{d t} Z_{\omega / 2}(t)=A_{E}(t)-Z_{\omega / 2}(t) Q
$$

Tеорема 2. Для $E>E_{C_{0}}\left(\|q\|_{r}\right)$

1) матрица $Q(E)=0$ для коллапсирующей щели и $Q($ E) нильпотентна в концевых точках щели;

2) для плотного множсества $G_{\delta}$ потенииалов $q$ в $\|q\|_{r}$-топологии существует $E>E_{C_{0}}\left(\|q\|_{r}\right)$ такое, что для этого $E$ уравнение (2) имеет неограниченное решение $Y_{\omega / 2}(t)$ и $\rho(E)$ не является ни диофантовым, ни рациональным.

ТеОрема 3. Для $E>E_{C_{0}}\left(\|q\|_{r}\right)$ справедливь следующие утвержсдения:

1) для плотного множества $G_{\delta}$ потенциалов $q$ в $\|q\|_{r}$-топологии пересечение $\sigma(\bar{L}) \cap[E, \infty]$ есть канторово множество;

2) ограничение спектра $\sigma(\bar{L})$ на интервал $(E, \infty)$ содержит только абсолютно непрерывную компоненту.

Ранее в работах [5], [26] было показано, что спектр $\sigma(\bar{L})$ в пересечении с интервалом $(E, \infty)$ при достаточно больших $E$ содержит абсолютно непрерьвную компоненту $\widetilde{\sigma}_{a c}(\bar{L})$ и мера $(\sigma(\bar{L}) \backslash \widetilde{\sigma}(\bar{L})) \cap(E, \infty) \rightarrow 0$ при $E \rightarrow \infty$.

Затем в [27] была построена другая часть абсолютно непрерьвного спектра $\widetilde{\sigma}(\bar{L}) \subset \sigma(\bar{L})$, не содержашаяся в $\widetilde{\sigma}(\bar{L})$. Однако, $\widetilde{\sigma}(\bar{L}) \cup \widetilde{\widetilde{\sigma}}(\bar{L})$ не совпадало со всем спектром.

В [5] и [26] впервые были применены методы КАМ-теории для анализа спектра квазипериодических операторов и доказательства приводимости системы (2) на Tor ${ }^{d}$ 
к системе с постоянными коэффициентами для значений $E$, принадлежаших спектру оператора $\sigma(\bar{L})$. Как известно, методы КАМ-теории предполагают отсутствие малых знаменателей (резонансов) на каждом шаге индуктивной процедуры, что приводит к бесконечному числу дополнительных условий на значения $E$, при которых этот процесс может быть проведен до конца. Этим объясняется то обстоятельство, что в результате применения КАМ-теории получается лишь часть спектра.

В [27] методы приводимости уравнения (2) были развиты для случая, когда на каждом шаге индукции имеется не более одного резонанса. В этом случае замена переменных в уравнении (2) уже не будет близкой к тождественной, как это было в [5] и [26]. Но присутствие не более одного резонанса на каждом шаге индуктивной процедуры и разреженность этих резонансов дает возможность доказать существование предельной замены, осуществляюшей приводимость уравнения (2).

Но и здесь при проведении индуктивной процедуры возникала необходимость исключения значений $E$, не удовлетворяющих бесконечному числу арифметических условий.

Основное достижение работы [24] состоит в том, что единственное арифметическое условие, ограничивающее значения $E$, для которых индукция может быть проведена, - это условие диофантовости или рациональности индекса вращения $\rho(E)$.

\section{Часть вторая. Экспоненциальная локализация собственных функций квазипериодических операторов с дальнодействием на решетке}

В этой части рассматриваются квазипериодические самосопряженные операторы на решетке, отвечаюшие взаимодействию бесконечного радиуса, убывающему экспоненщиально с ростом расстояния. К исследованию спектров таких операторов сводятся многие интересные физические задачи, например, движение электрона в постоянном магнитном поле при малых периодических возмущениях; распространение поверхностных волн в полупространстве, ограниченном шероховатой стенкой, форма которой задается квазипериодической функцией.

\section{$\S$ 1. Постановка задачи. Формулировка результата}

Как и в первой части, пусть $\mathbb{Z}^{\nu}$ есть $\nu$-мерная целочисленная решетка в $\mathbb{R}^{\nu}$, $n=\left(n_{1}, \ldots, n_{\nu}\right) \in \mathbb{Z}^{\nu}-$ произвольная точка решетки.

Для любой комплекснозначной функции $\varphi$ на решетке $\mathbb{Z}^{\nu}$ и любого действительного числа $\rho \geqslant 0$ определим $\rho$-норму функции $\varphi$ как

$$
\|\varphi\|_{\rho}=\sum_{n \in \mathbb{Z}^{\nu}}|\varphi(n)| e^{\rho|n|} .
$$

Удобно ввести в рассмотрение банахово пространство $H^{\rho}$ всех функций $\varphi$ с конечной $\rho$-нормой. Легко проверить, что для любых $\rho^{\prime} \geq \rho^{\prime \prime}$ справедливо неравенство $H^{\rho^{\prime}} \subseteq H^{\rho^{\prime \prime}}$.

Пусть $\left(X,\left\{T_{\omega}^{n}\right\}, \mu\right)$ - динамическая система, $X=S^{1}$ - единичная окружность, $\mu-$ мера Лебега, $\left\{T_{\omega}^{n}\right\}$ - действие групшы $\mathbb{Z}^{\nu}$ на $S^{1}$, определяемое следуюшим образом.

Зафиксируем вектор $\omega=\left(\omega_{1}, \ldots, \omega_{n}\right) \in \mathbb{R}^{\nu}$ с компонентами $\omega_{i}, 1 \leqslant i \leqslant \nu$, такой, что $\omega_{i}$ удовлетворяют условию: для любого $n \in \mathbb{Z}^{\nu}, n \neq 0$,

$$
|\{(n, \omega)\}|>0 .
$$


Здесь фигурные скобки обозначают дробную часть числа, $(n, \omega)=n_{1} \omega_{1}+\cdots+$ $n_{\nu} \omega_{\nu}$.

Положим для $x \in S^{1}$

$$
T_{\omega}^{n} x=x+(n, \omega) \quad(\bmod 1)
$$

Ясно, что динамическая система $\left(X,\left\{T_{\omega}^{n}\right\}, \mu\right)$ эргодична.

Теперь рассмотрим функцию $\varphi(n, x) \in A^{(\rho)}, \rho \geqslant 0, n \in \mathbb{Z}^{\nu}, x \in S^{1}$, и эргодическое семейство операторов $\widehat{\varphi}(x)$, определяемое функцией $\varphi(n, x)$ и ассоциированное с динамической системой $\left(X,\left\{T_{\omega}^{n}\right\}, \mu\right)$ (см. введение к этой статье).

Для любого $\varphi \in A^{\rho}$ мы введем разложение на его "диагональную" и "внедиагональную" части:

$$
\varphi(n, x)=D_{\varphi}(x) \delta_{n 0}+V_{\varphi}(n, x), \quad D_{\varphi}(x)=\varphi(0, x), \quad V_{\varphi}(n, x)=\left(1-\delta_{n 0}\right) \varphi(n, x) .
$$

Из определений следует, что в матричном представлении

$$
\widehat{\varphi}_{m n}(x)=D_{\varphi}\left(T^{n} x\right) \delta_{m n}+\left(1-\delta_{m n}\right) V_{\varphi}\left(n-m, T^{m} x\right),
$$

т.е. разложение $\varphi(n, x)$ на "диагональную" и "внедиагональную" компоненты связано с разложением оператора $\varphi(x)$ в сумму его диагональной и внедиагональной частей.

Напомним, что условие самосопряженности имеет вид

$$
\varphi^{*}(n, x)=\varphi(n, x)
$$

Основной результат этой части составляет

ТЕОремА. Пусть $d(x)-C^{2}$-функиия на $S^{1}$, имеющая ровно две критические невырожденные точки. Предположим также, что $V(n, x) \in A^{\rho}$ для некоторого $\rho>0 u V^{*}(n, x)=V(n, x), V(0, x) \equiv 0$. Тогда для любого $\delta>0$ существуют множество $\Omega_{\rho, \delta} \subseteq \Omega=[0,1]^{\nu}$, mes $\Omega_{\rho, \delta}>1-\delta$, и положительное число $\epsilon(d, \rho, \delta)$ такие, что если $\omega \in \Omega_{\rho, \delta},\|V\|_{\rho} \leqslant \epsilon(d, \rho, \delta),\left\|\frac{d V}{d x}\right\|_{\rho} \leqslant \epsilon(d, \rho, \delta),\left\|\frac{d^{2} V}{d x^{2}}\right\|_{\rho} \leqslant \epsilon(d, \rho, \delta)$, то для почти всех $x \in S^{1}$ операторы семейства $\widehat{\varphi}(x)$, определяемого функиией $\varphi(n, x)=d(x) \delta_{n 0}+\left(1-\delta_{n 0}\right) V(n, x)$ и ассоциированного с динамической системой $\left(X,\left\{T_{\omega}^{n}\right\}, \mu\right)$, имеют чисто точечный спектр. Все собственные функции этих операторов принадлежат пространству $H^{\rho \backslash 2}$. Кратность каждого собственного значения равна 1 . Спектр оператора $\widehat{\varphi}(x)$ как множество имеет положительную меру.

Ранее в [28] при тех же условиях было доказано, что для почти всех $x$ спектр оператора $\widehat{\varphi}(x)$ является чисто точечным, его собственные функции принадлежат $\ell^{1}\left(\mathbb{Z}^{\nu}\right) \subset \ell^{2}\left(\mathbb{Z}^{\nu}\right)$, кратность спектра не превосходит 2.

Для доказательства теоремы применяется индуктивный процесс, представляющий собой, как и в [28], вариант КАМ-теории для нерезонансных $x$ и процедуру расшепления резонансов для резонансных $x$, как и в [1]. Анализ резонансных значений $x$ основан на ограниченности кратности резонансов и их разреженности.

Усиление результата из [28] достигается благодаря иному выбору параметров индуктивного процесса и более точным оценкам приближенных собственных функций, строяшихся на каждом шаге индукции. 
ЗАмечАнИЕ. Пусть $e: S^{1} \rightarrow S^{1}$ - взаимно однозначное отображение окружности $S^{1}$, сохраняющее меру Лебега и переводящее каждую траекторию динамической системы $\left(S^{1},\left\{T_{\omega}^{n}\right\}, \mu\right)$ в себя. Последнее означает, что для любого $x \in S^{1}$ существует $p(x) \in \mathbb{Z}^{\nu}$, для которого $e(x)=T^{p(x)} x$. Рассмотрим произвольный самосопряженный элемент $\varphi(n, x) \in A^{\rho}$ и элемент $\widetilde{\varphi}(n, x)=\varphi(n, e(x))$. Легко проверить, что $\widetilde{\varphi}(n, x) \in A^{\rho}$ и $\|\varphi\|_{\rho}=\|\widetilde{\varphi}\|_{\rho}$.

Предположим, что $\left\{f_{n}(x)\right\}$ - собственный вектор оператора $\widehat{\varphi}(x)$ с собственным значением $\lambda(x)$, т.е.

$$
\sum_{n} \varphi\left(n-m, T^{m} x\right) f_{n}(x)=\lambda(x) f_{m}(x), \quad e(x)=T^{p(x)} x .
$$

Мы можем переписать это равенство следуюшим образом:

$$
\sum_{m} \varphi\left((n-p)-(m-p), T^{m-p}(e(x))\right) f_{m}(x)=\lambda(x) f_{m}(x) .
$$

Это означает, что оператор $\widehat{\varphi}(e(x))$ имеет собственный вектор $\left\{\bar{f}_{n}(x)\right\}=\left\{f_{n+p}(x)\right\}$ с тем же самьм собственным значением $\lambda(x)$. Таким образом, если оператор $\widehat{\varphi}(x)$ имеет полный набор собственных функций $\left\{f^{(n)}\right\}$ с собственными значениями $\left\{\lambda^{(n)}(x)\right\}$, то оператор $\widehat{\varphi}(e(x))$ также имеет полньй набор собственных функций $\left\{\bar{f}^{(n)}\right\}$ с теми же собственньми значениями. Из вида функции $\bar{f}^{(n)}$ следует, что она экспоненциально убывает при $|n| \rightarrow \infty$, если это верно для $f^{(n)}$ (множитель при экспоненте, вообще говоря, изменится). Это замечание будет неоднократно использовано в дальнейшем.

\section{§. Доказательство теоремы}

Как уже отмечалось, основой доказательства теоремы является индуктивньй процесс, которьй заключается в том, что на $s$-м шаге индукции мы определяем такой унитарный элемент $v^{(s)}(n, x)$ алгебры $A^{\left(\rho_{s}\right)}$, что элемент $\varphi^{(s+1)}(n, x)$, принадлежаший алгебре $A^{\left(\rho_{s+1}\right)}$ и равньй $\left(A d_{v^{(s)}}\left(\varphi^{(s)}\right)\right)(n, x)=\left(v^{(s)^{*}} \cdot \varphi^{(s)} \cdot v^{(s)}\right)(n, x)$, имеет вид

$$
\varphi^{(s+1)}(n, x)=d^{(s+1)}(x)+V^{(s+1)}(n, x),
$$

где элементы $d^{(s+1)}(x)$ и $V^{(s+1)}(n, x)$ являются кусочно непрерьвньми функциями от $x$ вместе с их первыми и вторьми производньми, элемент $V^{(s+1)}(n, x)$ имеет порядок малости $\epsilon^{(s+1)}, \epsilon^{(s+1)}=\left(\epsilon^{(s)}\right)^{1+\kappa}, \kappa$ - фиксированное положительное число. При этом функция $d^{(s+1)}(x)$ сохраняет свое основное свойство: каждое свое значение она принимает ровно два раза (с учетом кратности). Здесь и далее с целью сокращения обозначений, мы пишем $d(x), d^{(s)}(x)$ и т.п. вместо $d(x) \delta_{0 n}, d^{(s)}(x) \delta_{0 n}$ для обозначения диагональных частей элементов $\varphi(n, x), \varphi^{(s)}(n, x)$ алгебры $A^{\rho}$.

Величины $\rho_{s}$ убьвают с ростом $s$, оставаясь больше $\rho / 2$, что позволяет доказать экспоненциальное убьвание собственных функций исходного оператора $\widehat{\varphi}(x)$.

Более того, при фиксированном $x$ столбцы оператора $\widehat{\bar{v}}^{(s)}(n, x)=\left(v^{(1) \cdots v}(s)\right)(n, x)$ являются приближенными собственными функциями оператора $\widehat{\varphi}(n, x)$ с точностью $\epsilon^{(s+1)}$, а соответствующий набор приближенных собственных значений этого оператора совпадает с множеством значений функции $d^{(s+1)}$ вдоль траектории точки $x$ под действием динамической системы $\left\{T^{n}\right\}$. 
На $s$-м шаге индукции определяется понятие $s$-резонансной пары $(n, x)$, элемент $v^{(s)}(n, x)$ определяется различным образом для нерезонансных и резонансных значений $x$. Для первого случая $v^{(s)}(n, x)$ находится с помошью теории возмушений типа КАМ-теории. Для резонансных $x$, которым может отвечать только кратность 2 и соответствуюшие двумерные подпространства, элемент $v^{(s)}(n, x)$ соответствует вращению в таких подпространствах, приводяшему ограничение оператора $\widehat{\varphi}^{(s)}(n, x)$ на этих подпространствах к диагональному виду. Для таких $x$ элемент $v^{(s)}(n, x)$ не является близким к единичному в противоположность нерезонансным $x$. Такие врашения мы назьваем расшеплениями резонансных зон. В отличие от [1], где была доказана оценка снизу для убывания собственных функций и, благодаря этому, оценка снизу размера щели, образующейся в спектре при расщеплении внутри всей резонансной зоны, в нашем случае такая оценка, вообе говоря, не может быть получена, и мы вынуждены проводить расшепление лиш в части резонансной зоны. Поэтому мы различаем полное и частичное расшепление резонансных зон.

Однако, и в нашем случае почти каждая точка $x$ может находиться в резонансной зоне только конечное число раз. Это позволяет доказать сходимость $\prod_{s=1}^{\infty} \widehat{v}^{(s)}(n, x)$ для почти всех $x$, что обеспечивает существование полного набора собственных функций, обладаюшего желаемьми свойствами.

2.1. Индуктивная лемма. Положим $\kappa=1 / 4$ и определим следующие четыре последовательности чисел:

$$
\begin{gathered}
\epsilon_{s+1}=\epsilon_{s}^{1+\kappa}, \quad \epsilon_{1}=\epsilon, \quad R_{s}=\left[\Gamma s^{2} \ln \epsilon_{s}^{-1}\right], \quad \rho_{s+1}=\rho_{s}-\frac{3}{\pi^{2}} \frac{\rho}{s^{2}}, \quad s>1, \\
\rho_{1}=\rho, \quad \delta_{s}=\epsilon_{\bar{s}}^{\mu}, \quad \bar{s}=\max (1, s-g(s)),
\end{gathered}
$$

где

$$
g(s)=\left[(\ln \Gamma \rho+\ln 10+2 \ln s)(\ln (1+\kappa))^{-1}\right] .
$$

Здесь $\mu$ - достаточно малое число, $\Gamma$ - постоянная такая, что Г $\rho$ достаточно велико (можно считать, что $Г \rho=100)$.

Кроме того, мы предполагаем, что вектор $\omega$ удовлетворяет следующему диофантову условию:

$$
|\{(n, \omega)\}|>\frac{\epsilon^{\sigma}}{s^{2}|n|^{\nu+1}}, \quad|n| \leqslant R_{s}, \quad n \neq 0,
$$

с некоторой фиксированной постоянной $\sigma$ такой, что $\sigma / \mu$ достаточно мало. Здесь $\{\cdot\}$ обозначает дробную часть числа.

ЗАмечАнИЕ. Ясно, что $\rho_{s}>\rho / 2$. В самом деле,

$$
\rho_{s}=\rho-\frac{3}{\pi^{2}} \sum_{k=1}^{s} \frac{1}{s^{2}}>\rho-\frac{3}{\pi^{2}} \sum_{k=1}^{s} \frac{1}{s^{2}}=\frac{\rho}{2} .
$$

Смысл функции $g(s)$ станет ясным несколько позже. Ясно также, что лебегова мера множества векторов $\omega\left(\left|\omega_{i}\right|<1\right)$, удовлетворяюших условию $(D)$, больше $1-C \epsilon^{\sigma}$, где $C$ - абсолютная постоянная.

В дальнейшем в формулировках и доказательствах различных утверждений будут встречаться постоянные, как абсолютные, так и зависящие от вида функций $\varphi(n, x)$, 
размерности $\nu$ и т.п., но не зависящие от $s$. Все такие постоянные будут обозначаться символами $C, C_{1}, C_{2}, \ldots$, иногда даже различные постоянные будут обозначаться одним и тем же символом. В других случаях будет указываться зависимость от $s$ : $C(s), C_{1}(s), \ldots$. Значение $\epsilon$ предполагается столь мальм, что все необходимые соотношения вьполняются.

Предположим, что для $1 \leqslant t \leqslant s$

$\left.0_{s}\right)$ существуют такие множсества $\Omega_{t} \subset \Omega$, mes $\Omega_{t}>1-C \varepsilon^{\sigma} \sum_{k=1}^{t} k^{-2}$, $\Omega_{t} \supset \Omega_{t+1}$, что для $\omega \in \Omega_{t}$ выполнень следующие условия:

$\left.1_{s}\right) \varphi_{\omega}^{(t)}(n, x)=d_{\omega}^{(t)}(x)+V_{\omega}^{(t)}(n, x)$, где $d_{\omega}^{(t)}(x)=\varphi_{\omega}^{(t)}(0, x) ;$ функции $d_{\omega}^{(t)}(x)$, $V_{\omega}^{(t)}(n, x)$ кусочно непрерывны и на каждом интервале (полуинтервале, отрезке) непрерывности принадлежсат классу $C^{2}$; в точках непрерывности (односторонней непрерывности) функции $d_{\omega}^{(t)}(x)$ функции $V_{\omega}^{(t)}(n, x)$ непрерывны (односторонне непрерывны $)$, если $\bar{x}$ - точка разрыва функции $d_{\omega}^{(t)}(x)$ или $V_{\omega}^{(t)}(n, x)$, то существует такое $p(\bar{x}),|p(\bar{x})|<R_{t}$, что $T^{p(\bar{x})} \bar{x}$ является также точкой разрыва функиии $d_{\omega}^{(t)}(x)$, причем лево (право)-сторонние предельц функций $d_{\omega}^{(t)}(x)$ и $V_{\omega}^{(n)}(n, x)$ и их первых и вторых производных совпадают с право(лево)-сторонними пределами функиий $d_{\omega}^{(t)}\left(T^{p(\bar{x})} x\right), V_{\omega}^{(t)}\left(n, T^{p(\bar{x})} x\right)$ и их первьх и вторых производных при $x \rightarrow \bar{x}$.

(Элемент $\varphi_{\omega}^{(t)}(n, x)$ зависит от $\omega$, но мы далее опускаем указание на эту зависимость с целью упрощения обозначений, имея в виду, что соответствующие условия вьполнены для каждого $\omega \in \Omega_{t}$.)

Для формулировки остальных условий необходимо привести некоторые определения.

ОПРЕДЕЛЕНИЕ 1 . Пара $(n, x), x \in S^{1}, n \neq 0 \in \mathbb{Z}^{\nu}$, назьвается $t$-резонансной, если $|n| \leqslant R_{t}$ и

$$
\left\|d^{(t)}(x)-d^{(t)}\left(T^{n} x\right)\right\|<\delta_{t}
$$

ЗАмЕчАнИЕ. Очевидно, что вместе с $t$-резонансной парой $(n, x)$ таковой является пара $\left(-n, T^{n} x\right)$.

ОПРЕДЕЛЕНИЕ 2 . Связная компонента $O_{t, i}^{(n)}$ множества точек $x$, для которых $(n, x)$ является $t$-резонансной парой, называется резонансной зоной (РЗ) порядка $t$.

Здесь индекс $i$ отмечает номер РЗ при фиксированных $n$ и $t$; иногда мы будем опускать этот индекс, имея в виду, что соответствующие утверждения справедливы для любого возможного значения индекса $i$.

ОПРЕДЕЛЕНИЕ 3 . Связная компонента $\widetilde{O}_{t, i}^{(n)}$ множества

$$
\left\{x \in S^{1}:\left|d^{(t)}(x)-d^{(t)}\left(T^{n} x\right)\right|<2 \delta_{t}\right\}
$$

содержащая $\mathrm{P} 3 O_{t, i}^{(n)}$, называется расширением этой $\mathrm{P} 3$. 
$\left.2_{s}\right)$ Для каждого $t$ рассмотрим все $Р$ Р $O_{t_{1}, i}^{(n)}$ поряджа $t_{1}$, где $\bar{t} \leqslant t_{1} \leqslant t$. РЗ $O_{t_{1}, i}^{(n)}$ будет называться максимальной, если она не содерэстся ни в какой другой РЗ $O_{t_{2}, j}^{(m)}, \bar{t} \leqslant t_{2} \leqslant t_{1}$. Расширения $\widetilde{O}_{t_{1}, i}^{(n)}$ всех максимальных РЗ для $\bar{t} \leqslant t_{1} \leqslant t$ попарно не пересекаются. Диаметр расширения каждоймаксимальной РЗ порядка $t_{1}$ не превосходит $C \delta_{t_{1}}^{1 / 2}$. Число РЗ $O_{t_{1}, i}^{(n)}$ с фиксированным $n$ конечно.

В дальнейшем мы будем рассматривать только максимальные РЗ, не оговаривая этого специально.

$\left.3_{s}\right)$ Функции $d^{(t)}(x)$ и $V^{(t)}(n, x)$ таковьл, что их ограничения на $\widetilde{O}_{t_{1}}^{(n)}, \bar{t} \leqslant t_{1} \leqslant t$, принадлежат $C^{2}\left(\widetilde{O}_{t_{1}}^{(n)}\right)$.

$\left.4_{s}\right)$ Функиия $V^{(t)}(n, x)$ может быть представлена в виде суммы двух слагаeмubx:

$$
V^{(t)}(n, x)=V_{R}^{(t)}(n, x)+V_{N R}^{(t)}(n, x),
$$

где $V_{N R}^{(t)}(n, x)=V^{(t)}(n, x)$, если $(n, x)$ не является $t_{1}$-резонансной парой для $\bar{t} \leqslant$ $t_{1} \leqslant t, u V_{N R}^{(t)}(n, x)=0$ в остальных случаях. При этом $V_{N R}^{(t)}(n, x) \in A^{\left(\rho_{t}\right)} u$ имеют место следующие оценки:

$$
\left\|V_{N R}^{(t)}(n, x)\right\|_{t} \leqslant \epsilon_{t}, \quad\left\|\frac{d}{d x} V_{N R}^{(t)}(n, x)\right\|_{t} \leqslant \epsilon_{t}^{3 / 4}, \quad\left\|\frac{d^{2}}{d x^{2}} V_{N R}^{(t)}(n, x)\right\|_{t} \leqslant \epsilon_{t}^{1 / 2}
$$

(здесь и далее мы пишем $\|\cdot\|_{t}$ вместо $\left.\|\cdot\|_{\rho_{t}}\right)$.

Функиия $V_{R}^{(t)}(n, x)$ представляется в виде суммы $((t-\bar{t})+1)$ слагаемьх:

$$
V_{R}^{(t)}(n, x)=\sum_{l=0}^{t-\bar{t}} V_{R}^{(t), l}(n, x)
$$

где $V_{R}^{(t), l}(n, x)=V^{(t)}(n, x)$ для $x \in \bigcup_{i} O_{t-l, i}^{(n)} u V_{R}^{(t), l}(n, x)=0$ в остальньхх случаях. Справедливы следующие оченки:

$$
\left\|V_{R}^{(t), l}(n, x)\right\|_{t} \leqslant C \epsilon_{t-l}, \quad\left\|\frac{d}{d x} V_{R}^{(t), l}(n, x)\right\|_{t} \leqslant C \epsilon_{t-l}^{3 / 4}, \quad\left\|\frac{d^{2}}{d x^{2}} V_{R}^{(t), l}(n, x)\right\|_{t} \leqslant C \epsilon_{t-l}^{1 / 2} .
$$

$M$ мь будем называть $V_{R}^{(t)}(n, x)$ резонансной частью, $a V_{N R}^{(t)}(n, x)$ - нерезонансной частью әлемента $\varphi^{(t)}(n, x)$.

Для того чтобы сформулировать остальные условия, удобно ввести вспомогательные функции $\tau^{(t)}(x), \tau_{1}^{(t)}(x)_{1}$ и $\tau_{*}^{(t)}(x)$, определенные для всех $t \leqslant s$ и принимающие значения в множестве неотрицательных целых чисел.

Функция $\tau^{(t)}(x)$ отмечает попадание точки $x$ в РЗ: $\tau^{(t)}(x)=t$, если $x$ принадлежит PЗ порядка $t_{1}, \bar{t} \leqslant t_{1} \leqslant t$, и $\tau^{(t)}(x)=\tau^{(t-1)}(x)$ в противном случае. Мы полагаем $\tau^{(0)}(x) \equiv 0$.

Функция $\tau_{1}^{(t)}(x)$ связана с моментом первого попадания в РЗ, возможно после некоторого перерьва: $\tau_{1}^{(t)}(x)=t_{1}$, если $\tau^{(\widetilde{t})}(x)=\widetilde{t}$ для всех $t_{1} \leqslant \widetilde{t} \leqslant t$, но $\tau^{\left(t_{1}-1\right)}(x) \neq$ $t_{1}-1$. 
Функция $\tau_{*}^{(t)}(x)$ определяется более сложным образом, и ее точное определение будет дано в ходе доказательства индуктивной леммы. (Смысл функции $\tau_{*}^{(t)}(x)$ состоит в том, что $\tau_{*}^{(t)}(x)=t_{0}$, если на шаге индукции с номером $t_{0}$ произошло последнее расщепление РЗ, содержашей точку $x$.) Здесь важно отметить следующие ее свойства: $\tau_{*}^{(t)}(x)=\tau_{*}^{(t-1)}(x)$, если $x$ не принадлежит ни одной РЗ порядка $t_{1}, \bar{t} \leqslant t_{1} \leqslant t$.

$\left.5_{s}\right)$ Пусть $\tau_{1}^{(t)}(x)=t u x \in O_{t}^{(n)}$. Тогда существует такая точка $x_{*} \in O_{t}^{n}$, что $d^{(t)}\left(x_{*}\right)=d^{(t)}\left(T^{n} x_{*}\right) u \operatorname{sgn} \frac{d}{d x} d^{(t)}\left(x_{*}\right)=-\operatorname{sgn} \frac{d}{d x} d^{(t)}\left(T^{n} x_{*}\right)$ для всех $x \in O_{t}^{(n)}$. Eсли $\tau_{*}^{(t-1)}(x)>0$, mo $t-\tau_{*}^{(t-1)}(x)>C \ln \epsilon^{-1}(1+\kappa)^{\overline{\tau_{*}^{(t-1)}}}(x)$. Cправедливь следующие неравенства:

$$
\left|\frac{d}{d x} d^{(t)}(x)\right|<C, \quad\left|\frac{d^{2}}{d x^{2}} d^{(t)}(x)\right|<C \frac{t-t_{*}^{(t-1)}(x)+1}{\epsilon_{\tau_{*}^{(t-1)}(x)+1}} .
$$

Кроме того, либо $\left|\frac{d}{d x} d^{(t)}(x)\right|>C \epsilon_{\tau_{*}^{(t-1)}(x)+1}$, либо $\left|\frac{d^{2}}{d x^{2}} d^{(t)}(x)\right|>C$, причем первое неравенство может нарушаться только в окрестности критической точки функции $d^{(t)}(x)$ диаметра $C \epsilon_{\tau_{*}^{(t-1)}(x)+1}$, в которой второе неравенство выполнено.

Пусть $x^{*}$ - критическая точка функции $d^{(t)}(x) u \tau_{*}^{(t)}(x)=t_{1}<t$. Тогда существуют такие постоянные $C_{1}>0, C_{2}>0$ и такая окрестность $O_{t_{1}}\left(x^{*}\right)$ точки $x^{*}$, что функиия $d^{(t)}(x)$ каждое свое значение $\bar{d},\left|\bar{d}-d^{(t)}\left(x^{*}\right)\right|<C_{1} \delta_{t_{2}}^{1 / 2}$, принимает ровно два раза (с учетом кратности), при этом $C_{2} \delta_{t_{2}}<\operatorname{diam}\left(O_{t_{1}}\left(x^{*}\right)\right)<$ $C_{2} \delta_{t_{2}}^{1 / 2}$. Здесь $t_{2}=\max \left(k: t>k \geqslant t_{1}, O_{t_{1}}^{(n)}\left(x^{*}\right) \cap O_{k}^{(m)} \neq \varnothing\right), O_{t_{1}}^{(n)}\left(x^{*}\right)-$ РЗ порядка $t_{1}$, содерэсащая точку $x^{*}$.

$6_{s}$ ) Функция $d^{(t)}(x)$ принимает каждое свое значение ровно два раза (с учетом кратности).

Пусть $I_{t}$ - область значений функции $d^{(t)}(x)$ и $I_{t}=\cup I_{t}^{(i)}$ - ее разложение на связные компоненты. Положим $S_{t}^{(i)}=\left(d^{(t)}\right)^{-1}\left(I_{t}^{(i)}\right)$ для каждого $i$. При любом $i S_{t}^{(i)}$ состоит не более чем из двух компонент, каждая из которых является полуинтервалом. Если $S_{t}^{(i)}$ состоит из двух компонент, скажем, $S_{t, 1}^{(i)}$ u $S_{t, 2}^{(i)}$, mо существует такое $p=p\left(S_{t}^{(i)}\right)$, ито $|p|<R_{\bar{s}}, T^{p} S_{t, 2}^{(i)} \cap S_{t, 1}^{(i)}=\varnothing, a$ $S_{t, 1}^{(i)} \cup T^{p} S_{t, 2}^{(i)}=\widetilde{S}_{t}^{(i)}$ представляет собой полуинтервал.

B случае связного $S_{t}^{(i)}$ положим $S_{t, 1}^{(i)}=S_{t}^{(i)}, S_{t, 2}^{(i)}=\varnothing$.

Функиия $\widetilde{d}^{(t)}(x)$, равная $d^{(t)}(x)$ на $S_{t, 1}^{(i)}$ и $d^{(t)}\left(T^{-p} x\right)$ на $T^{p} S_{t, 2}^{(i)}$, непрерьвна вместе со своими первыми двумя производными, имеет ровно две невьрожденные критические точки на $\widetilde{S}_{t}^{(i)}$ и принимает каждое свое значение (за исключением значения в коние полуинтервала) ровно два раза (с учетом кратности).

Если $x$ не содержится в расширении ни одной РЗ $\widetilde{O}_{t_{1}}^{(n)}$ ини одного из сдвигов $T^{k} \widetilde{O}_{t}^{(n)},|k| \leqslant R_{\bar{t}}, \bar{t} \leqslant t_{1} \leqslant t$, то $\left|d^{(t-1)}(x)-d^{(t)}(x)\right|<\epsilon_{t} \epsilon_{\bar{t}}^{-\mu}$. На кажсдом максимальном интервале непрерывности $d^{(t)}(x)$ содержит, по крайней мере, 
одну критическую точку. Внутри каждой $Р$ З $O_{t_{1}}^{(n)}, \bar{t} \leqslant t_{1} \leqslant t$, функиия $d_{1}^{(t)}(x)$ или строго монотонна, или имеет ровно одну невырожденную критическую точку. В первом случае внутри $Р З O_{t_{1}}^{(n)}$ существует точка $x_{*}$, в которой $d^{(t)}\left(x_{*}\right)=d^{(t)}\left(T^{n} x_{*}\right)$.

Во втором случае пусть $O_{t_{1}}^{(n)}-P 3$, содержащая точку $x, a O_{t_{1}}^{(-n)}-P З$, содержащая точку $T^{n} x, t_{1}=\tau_{*}^{\left(t_{1}\right)}(x)+1$. Положим $I_{t_{1}}^{(n)}=\left(d^{\left(t_{1}\right)}\right)^{-1}\left(O_{t_{1}}^{(n)}\right)$, $I_{t_{1}}^{(-n)}=\left(d^{\left(t_{1}\right)}\right)^{-1}\left(O_{t_{1}}^{(-n)}\right)$. Tогдa $C_{2} \epsilon_{\overline{t_{1}-1}}>\operatorname{dist}\left(I_{t_{1}}^{(n)}, I_{t_{1}}^{(-n)}\right)>C_{1} \epsilon_{t_{1}}$.

ИнДУКТИВНАя ЛЕМма. Пусть выполнены предположсения $\left.0_{s}\right)-6_{s}$ ). Тогда существуют такой унитарный элемент $u^{(s)} \in A^{\rho_{s}}$ и такое взаимно однозначное сохраняющее меру Лебега и переводящее каждую траекторию динамической системы $\left(S^{1},\left\{T_{\omega}^{n}\right\}, \mu\right)$ в себя отображсение $e^{(s)}: S^{1} \rightarrow S^{1}$, что

$$
\varphi^{(s+1)}(n, x)=\bar{\varphi}^{(s+1)}\left(n, e^{(s)}(x)\right)
$$

удовлетворяет предположсениям $\left.\left.0_{s+1}\right)-6_{s+1}\right)$, где

$$
\bar{\varphi}^{(s+1)}(n, x)=u^{(s)^{*}} \varphi^{(s)} u^{(s)}(n, x) .
$$

Кроме того, $u^{(s)}(n, x)=q^{(s)} r^{(s)}(n, x)$, где $q^{(s)}(n, x), r^{(s)}(n, x) \in A^{\rho_{s}}$ - унитарние әлементы,$\left\|q^{(s)}-I d\right\|_{\rho_{s}}<C \varepsilon_{s} \varepsilon_{\bar{s}}^{-\mu}$, әлементы $r^{(s)}(n, x)$ совпадают с единичным вне облединения расширений $Р 3$ порядка $t, \bar{s} \leqslant t \leqslant s$.

Отображсние $e^{(s)}: S^{1} \rightarrow S^{1}$ может быть описано следующим образом. Существуют конечное множество индексов $J^{(s)}$, его разбиение $J^{(s)}=J_{1}^{(s)} \cup J_{2}^{(s)}$, $J_{1}^{(s)} \cap J_{2}^{(s)}=\varnothing,\left|J_{1}^{(s)}\right|=\left|J_{2}^{(s)}\right|$, конечное число подмножеств $I_{j}$, занумерованных әлементами множества $J^{(s)}$, взаимно однозначное отображсение $l: J_{1}^{(s)} \rightarrow J_{2}^{(s)}$ и функиия $p_{s}: S^{1} \rightarrow \mathbb{Z}^{\nu}$ такие, что

1) каждое из множеств $I_{j}$ является интервалом или полуинтервалом;

2) $I_{j_{1}} \cap I_{j_{2}}=\varnothing$, если $j_{1} \neq j_{2}$;

3) каждое множество $I_{j}$ содержится либо в расширении какой-либо РЗ $\widetilde{O}_{s+1}^{(n)}$ порядка $s+1$ для $\varphi^{(s+1)}(n, x)$, либо в ее сдвиге $T^{k} \widetilde{O}_{s+1}^{(n)},|k|<R_{\bar{s}}$, $p_{s}(x)=0 \quad$ для $x \in S^{1} \backslash \bigcup_{j \in J^{(s)}} I_{j} ; p_{s}(x)$ постоянна на $I_{j}, j \in J^{(s)}$; $\left.p_{s}(x)\right|_{I_{j_{1}}}=-\left.p_{s}(x)\right|_{I_{l\left(j_{1}\right)}}, e^{(s)}(x)=T^{p_{s}(x)} x$.

ЛЕМма 1. Для $s=1$ индуктивные предположения $\left.0_{s}\right)-6_{s}$ ) выполнены.

ДОКАЗАТЕЛЬСТВО. Положим $\tau^{(1)}(x)=1$ для окрестностей критических точек (максимум и минимум) диаметра $\epsilon_{1}^{\mu / 2}$ и $\tau^{(1)}(x)=0$ для остальных $x$. Кроме того, $\tau_{1}^{(1)}(x)=\tau^{(1)}(x)$ и $\tau_{*}^{(1)}(x)=\tau^{(1)}(x)$. В качестве множества $\Omega_{1}$ выберем множество всех таких $\omega$, что $|\{(n, \omega)\}|>\epsilon^{\sigma} /|n|^{\nu+1}$ для $|n| \leqslant R_{1}, n \neq 0$. Теперь выполнение всех индуктивных предположений может быть проверено непосредственно.

Доказательство индуктивной леммы будет приведено в $\S 3$. 


\section{2. Вывод теоремы из индуктивной леммы.}

Лемма 2. 1) Почти каждая точка $x \in S^{1}$ принадлежит множествам

$$
O^{(s)}=\bigcup_{\bar{s} \leqslant t \leqslant s} \bigcup_{n} \bigcup_{i} O_{t, i}^{(n)}
$$

лишь для конечного числа значений $s$.

2) Почти каждая точка $x \in S^{1}$ принадлежит множествам

$$
\widetilde{O}^{(s)}=\bigcup_{\bar{s} \leqslant t \leqslant s} \bigcup_{n} \bigcup_{i} \widetilde{O}_{t, i}^{(n)}
$$

лишь для конечного числа значений $s$.

3) Положим $A_{s}=\left\{x \in S^{1}: e^{(s)}(x) \neq x\right\}, 1 \leqslant s<\infty$. Почти каждая точка $x \in S^{1}$ принадлежит множествам $A_{s}$ лишь для конечного числа значений $s$.

ДокАЗАтЕльство. Сначала докажем утверждение 1). Мера РЗ $O_{t}^{(n)}$ порядка $t$, $\bar{s} \leqslant t \leqslant s$, не превосходит $C \delta_{\bar{s}}^{1 / 2}$. Каждая точка $x$ находится в Р $O_{t}^{(n)}$ не более $g(s)$ шагов индукции. Число различных $n \mathrm{c}|n| \leqslant R_{s}$ не превосходит $C R_{s}^{\nu}$.

Оценим сверху число РЗ $O_{t}^{(n)}$ с фиксированным $n$. Каждая РЗ в момент своего возникновения $\tau_{1}$ содержит такую точку $x_{*}$, что $d^{\left(\tau_{1}\right)}\left(x_{*}\right)=d^{\left(\tau_{1}\right)}\left(T^{k} x_{*}\right)$, где $|k| \leqslant R_{\tau_{1}}$ и функции $d^{\left(\tau_{1}\right)}(x)$ и $d^{\left(\tau_{1}\right)}\left(T^{k} x\right)$ имеют противоположные монотонности в окрестности точки $x_{*}$. Следовательно, число РЗ $O_{s}^{(n)}$ с фиксированньм $n$ не превосходит числа критических точек функции $d^{(s)}(x)$, которое, в свою очередь, не превосходит числа всех РЗ порядка $t \leqslant s$. Поэтому если число РЗ порядка $\leqslant k-1$ равно $N_{k-1}$, то число $N_{k}^{(n)}$ РЗ порядка $k$ с фиксированньм $n$ не превосходит $N_{k-1}$. Отсюда получаем, что $N_{k}<C N_{k-1} R_{k}^{\nu}$. Так как $N_{0}=2$, то $N_{k} \leqslant 2 C^{k} \prod_{l=1}^{k} R_{l}^{\nu}$. Итак, сумма мер множеств точек $x$, принадлежаших на $s$-м шаге индукции какой-либо РЗ, по всем $s$ не превосходит

$$
\sum \delta_{\bar{s}}^{1 / 2} \cdot g(s) \cdot N_{s} R_{s}^{\nu}<2 \sum_{s}\left(\delta_{\bar{s}}^{1 / 2} \cdot g(s) \cdot C^{s} \prod_{k=1}^{s} R_{k}^{\nu}\right)<\infty
$$

Из леммы Бореля-Кантелли следует, что почти каждая точка $x$ окружности $S^{1}$ принадлежит РЗ не более конечного числа шагов индукции.

Утверждение 2) доказывается аналогично.

Для доказательства утверждения 3) достаточно заметить, что $A_{s}$ содержится в объединении расширений всех РЗ $\widetilde{O}_{s+1, i}^{(n)}$ порядка $s+1$ и их сдвигов $T^{k} \widetilde{O}_{s+1, i}^{(n)},|k| \leqslant R_{\bar{s}}$. Следовательно, $\mu\left(A_{s}\right) \leqslant C_{1} R_{\bar{s}}^{\nu} \cdot N_{s+1} \cdot C \varepsilon_{\bar{s}}^{\mu / 2}$, где $N_{s+1}$ - число РЗ порядка $s+1$. Как и в доказательстве утверждения 1 ), получаем, что

$$
\sum_{s} \mu\left(A_{s}\right)<C(\varepsilon)<\infty
$$

где $C(\varepsilon) \rightarrow 0$ при $\varepsilon \rightarrow 0$. 
СлеДСТВИЕ 1. Положим $e_{s}=\prod_{k=1}^{s} e^{(k)}: S^{1} \rightarrow S^{1}$. Для почти каждой точки $x \in S^{1}$ существует такое $s_{0}(x)$, что $e_{s+1}(x)=e_{s}(x)$ для $s \geqslant s_{0}(x)$.

ДокаЗАТЕЛЬСТво. Положим $B_{s}=\left\{x \in S^{1}: e_{s+1}(x) \neq e_{s}(x)\right\}$. Так как $e_{s+1}=$ $e^{(s+1)} e_{s}$ и $e^{(k)}$ сохраняет меру $\mu, \mu\left(B_{s}\right)=\mu\left(A_{s}\right)$. Из $\sum_{k} \mu\left(A_{k}\right)<\infty$ вытекает справедливость следствия.

ЗАмЕчАнИЕ. Следствие 1 позволяет определить отображение $e_{\infty}: S \rightarrow S$, где $S \subset S^{1}$ и $\mu(S)=1$. А именно, для любой точки $x$, удовлетворяюшей следствию 1 , положим $e_{\infty}(x)=e_{\bar{s}_{0}(x)}(x)$, где $\bar{s}_{0}(x)=\min s_{0}(x)$. Можно считать, что $S$ инвариантно относительно $\left\{T_{\omega}^{n}\right\}$, так как в противном случае можно вместо $S$ рассмотреть $\bigcap_{n \in \mathbb{Z}^{\nu}} T_{\omega}^{n} S$. Отображение $e_{\infty}: S \rightarrow S$ переводит каждую траекторию $\left\{T_{\omega}^{n} x\right\}$ в себя: $e_{\bar{s}_{0}(x)}(x)=\left(\prod_{k=1}^{\bar{s}_{0}(x)} e^{(k)}\right)(x)=T^{q(x)} x$, где $q(x)=\sum_{k=1}^{\bar{s}_{0}(x)} p_{k}\left(e_{k-1}(x)\right)$. Покажем, что отображение $e_{\infty}$ взаимно однозначно и сохраняет меру $\mu$. В самом деле, если $e_{\infty}\left(x_{1}\right)=e_{\infty}\left(x_{2}\right), x_{1} \neq x_{2}$, то $e_{\bar{s}_{0}\left(x_{1}\right)}\left(x_{1}\right)=e_{\bar{s}_{0}\left(x_{2}\right)}\left(x_{2}\right)$. Тогда $e_{\bar{s}}\left(x_{1}\right)=e_{\bar{s}}\left(x_{2}\right)$, где $\bar{s}=\max \left(\bar{s}_{0}\left(x_{1}\right), \bar{s}_{0}\left(x_{2}\right)\right)$, что невозможно, так как $e_{\bar{s}}$ взаимно однозначно. Пусть $B \subset S$ - измеримое множество, $C_{k} \subset S, C_{k}=\left\{x \in S: \bar{s}_{0}(x)=k\right\}, C_{k_{1}} \cap C_{k_{2}}=\varnothing$ при $k_{1} \neq k_{2}, \bigcup C_{k}=S$. Тогда $B=\bigcup_{k}\left(B \cap C_{k}\right)$ и $e_{\infty}(B)=\bigcup_{k} e_{k}\left(B \cap C_{k}\right)$. Из взаимнооднозначности отображения $e_{\infty}$ на $S$ следует, что множества $e_{\infty}\left(B \cap C_{k}\right)=e_{k}\left(B \cap C_{k}\right)$ попарно не пересекаются. Так как каждое отображение $e_{k}$ как конечное произведение отображений $e^{(l)}$ сохраняет меру $\mu$, получаем $\mu\left(e_{\infty}(B)\right)=\sum_{k} \mu\left(e_{k}\left(B \cap C_{k}\right)\right)=$ $\sum_{k} \mu\left(B \cap C_{k}\right)=\mu(B)$, что и требовалось доказать.

Лемма 3. Существует множсество $S_{2} \in S^{1}$ полной меры такое, что для любого $x \in S_{2}$ оператор $\widehat{\varphi}(n, x)$ имеет чисто точечный спектр, его собственные функиии $\widehat{\psi}_{i}(n, x), i \in \mathbb{Z}^{\nu}$, убывают бистрее, чем $C_{i}(x) \epsilon^{-\frac{\rho}{2}|n|}$. Кратность спектра равна 1.

ДокАЗАТЕЛЬСТВО. Положим $p^{(s)}(n, x)=\left(v^{(1)} \cdot v^{(2)} \cdots v^{(s)}\right)(n, x)$, где $v^{(k)}(n, x)=$ $u^{(k)}\left(n,\left(\prod_{t=k}^{s} e^{(t)}\right)(x)\right)$. Мы получаем, что

$$
\varphi^{(s+1)}(n, x)=\left(p^{(s)^{*}} \bar{\varphi}^{(s)} p^{(s)}\right)(n, x)
$$

с $\bar{\varphi}^{(s)}(n, x)=\varphi\left(n, e_{s}(x)\right)$. Непосредственно проверяется, что если $u(n, x)$ - унитарньй элемент алгебры $A^{\rho}$, то $u\left(n, e^{(s)}(x)\right)$ при любом $s$ является унитарным элементом алгебры $A^{\rho}$ с той же $\rho$-нормой. Поэтому $p^{(s)}(n, x)$ - унитарный элемент алгебры $A^{\left(\rho_{s}\right)}$.

Рассмотрим множество $S_{0}$ всех точек $x \in S^{1}$, удовлетворяющих утверждениям 2) и 3) леммы 2. Положим $S_{1}=\bigcap_{n \in \mathbb{Z}^{\nu}} T^{n} S_{0}$. Ясно, что $\mu\left(S_{1}\right)=1$. Покажем, что для любого $x \in S_{1}$ существует $\lim _{s \rightarrow \infty} d^{(s)}(x)=d^{(\infty)}(x)$, где $d^{(s)}(x)=\varphi^{(s)}(0, x)$. В самом деле, если $x \in S_{1}$, то найдется такое $s_{1}(x)$, что при $s \geqslant s_{1}(x)$ точка $x$ не принадлежит расширениям $\widetilde{O}_{t}^{(n)}, \bar{s} \leqslant t \leqslant s+1$, и никаким $\bigcup_{|k|<R_{\bar{s}}} T^{k} \widetilde{O}_{s+1}^{(n)}$. В силу предположения $\left.6_{s}\right)\left|d^{(s+1)}(x)-d^{(s)}(x)\right|<\varepsilon_{s} \varepsilon_{\bar{s}}^{-\mu}$ и, следовательно, указанньй предел сушествует для $x \in S_{1}$, так как $S_{1} \subset S$ (S- множество точек $x$, удовлетворяюших утверждению 3) леммы 2).

Рассмотрим оператор $\widehat{p}^{(s)}(n, x)$. Покажем, что для почти каждого $x$ и любого $m \in \mathbb{Z}^{\nu}$ предел $\lim _{s \rightarrow \infty} p_{m}^{(s)}(n, x)=p_{m}^{(\infty)}(n, x)$ существует в $\ell^{2}\left(\mathbb{Z}^{\nu}\right)$, где $p_{m}^{(s)}(x)$ - $m$-й столбец матрищы $\widehat{p}^{(s)}(x)$, и матрица $\widehat{p}^{(\infty)}(x)$, составленная из столбцов $p_{m}^{(\infty)}(x)$, есть матрица унитарного оператора в $\ell^{(2)}\left(\mathbb{Z}^{\nu}\right)$. Действительно, элементы 
$p_{m}^{(s)}(x)$ имеют вид $p^{(s)}\left(m-n, T^{n} x\right)$. Если $x \in S_{1}$, то $T^{n} x \in S_{1}$ для любого $n \in \mathbb{Z}^{\nu}$ в силу инвариантности множества $S_{1}$. Более того, рассмотрим множества $C_{s}^{(m)}=\left\{x \in S^{1}: T^{n} x \in \widetilde{O}^{(s)}\right.$ для некоторого $\left.n,|m-n| \leqslant R_{s}\right\}$ и множества $B_{s}^{(m)}=\left\{x \in S^{1}: T^{n} x \in A_{s}\right.$ для некоторого $\left.n,|m-n|<R_{s}\right\}$. Как и в доказательстве леммы 2 , можно показать, что при достаточно малом $\varepsilon$ выполняется $\sum \mu\left(B_{s}^{(m)}\right)<\infty$ и $\sum \mu\left(C_{s}^{(m)}\right)<\infty$ и, следовательно, для почти каждой точки $x$ найдется такое $N(m, x)$, что $x \notin \bigcup_{s>N(m, x)}\left(C_{s}^{(m)} \cup B_{s}^{(m)}\right)$. Обозначим множество таких $x$ через $S_{1}^{(m)}$ и положим $S_{2}=S_{1} \cap\left(\bigcap_{m} S_{1}^{(m)}\right)$. Ясно, что $\mu\left(S_{2}\right)=1$. Тогда для любого $x \in S_{2}$ имеем

$$
p_{m}^{(s)}(x)=\left(\prod_{t>t_{1}>N(m, x)}^{s} \widehat{q}^{(t)}(x)\right) p_{m}^{\left(t_{1}\right)}(x) .
$$

Так как при любом $x \in S_{2}$ произведение $\left(\prod_{t>t_{0}} q^{(t)}\right)(n, x)$ сходится в $\rho / 2$-норме, то $\lim _{s \rightarrow \infty} p_{m}^{(s)}(x)=p_{m}^{(\infty)}(x)$ сушествует,

$$
p_{m}^{(\infty)}(x)=\left(\prod_{t>N(m, x)}\left(\widehat{q}^{(t)}(x)\right)\right) p_{m}^{(N(m, n))}(x)
$$

и компоненты $p_{m}^{(\infty)}(n, x)$ вектора $p_{m}^{(\infty)}(x)$ удовлетворяют неравенству

$$
\left|p_{m}^{(\infty)}(n, x)\right|<C(m, x) e^{-\frac{\rho}{2}|n|}
$$

при некоторой постоянной $C(m, x)$.

Для различных $m_{1}, m_{2} \in \mathbb{Z}^{\nu}$ векторы $p_{m_{1}}^{(\infty)}(x)$ и $p_{m_{2}}^{(\infty)}(x)$ ортогональны, и их совокупность $\left\{p_{m}^{(\infty)}(x)\right\}, m \in \mathbb{Z}^{\nu}$, образует ортонормированный базис в $\ell^{2}\left(\mathbb{Z}^{\nu}\right)$. Это вытекает из унитарности операторов $\widehat{p}^{(s)}(x)$ при любом конечном $s$. Легко проверить, что $p_{m}^{(\infty)}(x)$ является собственным вектором оператора $\widehat{\varphi}\left(e_{\infty}(x)\right)$ с собственньм значением $d^{(\infty)}\left(T^{m} x\right)$. Из замечания в конце $\S 1$ следует, что оператор $\widehat{\varphi}(x)$ имеет полный набор $\left\{\psi_{m}(x)\right\}$ собственных экспоненциально убывающих функций $\psi_{m}(x)$ с собственными значениями $d^{(\infty)}\left(T^{m} x\right), m \in \mathbb{Z}^{\nu}$.

Осталось доказать утверждение о кратности спектра.

Предположим, что $d^{(\infty)}(\bar{x})=d^{(\infty)}\left(T^{k} \bar{x}\right)$ для $\bar{x} \in S_{2}$, и покажем, что это равенство невозможно. Пусть $s^{*}$ таково, что точки $\bar{x}$ и $T^{k} \bar{x}$ не принадлежат расширению ни одной РЗ $\widetilde{O}_{s}^{(n)}$ и ни одному $\bigcup T^{k} \widetilde{O}_{s+1}^{(n)},|k|<R_{\bar{s}}$ для $s \geqslant s^{*}+1$. Следовательно, $\left|d^{\left(s^{*}+1\right)}(\bar{x})-d^{\left(s^{*}+1\right)}\left(T^{k} \bar{x}\right)\right|>\epsilon_{\frac{\mu}{s^{*}+1}}^{\mu}\left|d^{(s)}(\bar{x})-d^{(s+1)}(\bar{x})\right|<\epsilon_{s} \epsilon_{\bar{s}}^{-\mu}$, $\left|d^{(s)}\left(T^{k} \bar{x}\right)-d^{(s+1)}\left(T^{k} \bar{x}\right)\right|<\epsilon_{s} \epsilon_{\bar{s}}^{-\mu}$ для $s>s^{*}$. Но $\sum_{s>s^{*}} \epsilon_{s} \epsilon_{\bar{s}}^{-\mu}<C \epsilon_{s^{*}+1} \epsilon_{s^{*}+1}^{\mu}$, поэтому $\left|d^{(s)}(\bar{x})-d^{(s)}\left(T^{k} \bar{x}\right)\right|>\frac{1}{2} \epsilon_{s^{*}+1}^{\mu}$ для $s>s^{*}$. Отсюда вытекает, что

$$
\left|d^{(\infty)}(\bar{x})-d^{(\infty)}\left(T^{k} \bar{x}\right)\right|>\frac{1}{2} \epsilon_{s^{*}+1}^{\mu}, d^{(\infty)}(\bar{x}) \neq d^{(\infty)}\left(T^{k} \bar{x}\right),
$$

что и требовалось доказать.

Утверждение о мере спектра оператора $\widehat{\varphi}(n, x)$ для почти всех $x$ вытекает из того, что семейство операторов $\widehat{\varphi}(n, x)$ является метрически транзитивным и его спектр как множество не зависит от $x$ (см. [6]), и оценок шелей в спектре, вытекаюших из условия $6_{s}$ ). Лемма доказана. 


\section{§3. Доказательство индуктивной леммы}

Итак, мы предполагаем, что на $s$-м шаге индукции для всех $1 \leqslant t \leqslant s$ заданы

$$
\varphi^{(t)}(n, x)=d^{(t)}(x)+V_{R}^{(t)}(n, x)+V_{N R}^{(t)}(n, x),
$$

удовлетворяющие условиям $\left.0_{s}\right)-6_{s}$ ).

При переходе от $s$ к $s+1$ мы будем вьполнять различные преобразования над резонансной частью $V_{R}^{(s)}(n, x)$ элемента $\varphi^{(s)}(n, x)$ и его нерезонансной частью $V_{N R}^{(s)}(n, x)$. Эти преобразования будут отличаться в окрестности границ РЗ. Для того, чтобы избежать возникновения большого числа разрывов функции $\varphi^{(s+1)}(n, x)$ и ее производных, а также обеспечить сохранение основного свойства функции $d^{(s+1)}(x)$ принимать каждое свое значение ровно два раза (с учетом кратности), мы включаем в наш индуктивный процесс процедуру сглаживания, сопрягающую различные преобразования в точках их совместного применения.

3.1. Процедура сглаживания. Мы определяем вспомогательную функцию $F(x) \in C^{2}([0,1])$ и фиксируем ее с этого момента. Мы предполагаем, что вьполнены следуюшие условия:

1) $F(0)=0, F(1)=1$;

2) $F^{\prime}(0)=F^{\prime \prime}(0)=F^{\prime}(1)=F^{\prime \prime}(1)=0$;

3) $F^{\prime}(x)>0$ для $x \neq 0,1$.

Для любого интервала $\Delta=\left(x_{1}, x_{2}\right)$ положим $F_{\Delta}(x)=F\left(\frac{x-x_{1}}{|\Delta|}\right)$ при $x \in \Delta$, где $|\Delta|$ - длина интервала $\Delta$.

Каждая РЗ $O_{t}^{(n)}$ есть некоторьй интервал $\Delta_{t}^{(n)}=\left(x_{1}, x_{2}\right)$ на окружности $S^{1}$, $x_{1}<x_{2}$. (Мы предполагаем, что в качестве локальной координаты на $S^{1}$ выбрана длина дуги, отсчитьваемая от такой точки, что $x$ является однозначной функцией на $\Delta_{t}^{(n)}$.)

Для любой $\mathrm{P} 3 O_{t}^{(n)}=\left(x_{1}, x_{2}\right)$ рассмотрим два интервала $\Delta_{t}^{(n), 1}=\left(x_{1}, x_{1}^{\prime}\right)$ и $\Delta_{t}^{(n), 2}=\left(x_{2}^{\prime}, x_{2}\right),\left|\Delta_{t}^{(n), 1}\right|=\left|\Delta_{t}^{(n), 2}\right|=\varepsilon_{t}^{1 / 4}$.

Оба эти интервала принадлежат $\Delta_{t}^{(n)}$ и не пересекаются между собой, так как из ограниченности $\frac{d}{d x} d^{(s)}(x)$ вытекает, что $\left|\Delta_{t}^{(n)}\right|>C \varepsilon_{\frac{\mu}{t}}^{\mu}$. Теперь определим функшию $F_{t, n}^{(0)}(x)$ следуюшим образом: $F_{t, n}^{(0)}(x) \equiv 0$ вне $O_{t}^{(n)}, F_{t, n}^{(0)}(x)=F_{\Delta_{t}^{(n), 1}}(x)$ для $x \in \Delta_{t}^{(n), 1}, F_{t, n}^{(0)}(x)=1-F_{\Delta_{t}^{(n), 2}}(x)$ для $x \in \Delta^{(n), 2}(x), F_{t, n}^{(0)} x=1$ для $x \in O_{t}^{(n)}$ $\left(\Delta_{t}^{(n), 1} \cup \Delta_{t}^{(n), 2}\right)$.

Положим $F_{s}^{(0)}(x)=\sum F_{t, n}^{(0)} x$, где суммирование проводится по всем РЗ порядка $t$, $\bar{s} \leqslant t \leqslant s$. Из $\left.22_{s}\right)$ следует, что $F_{s}^{(0)}(x)$ корректно определена.

Представим $V^{(s)}(n, x)$ в виде суммы двух слагаемых

$$
V^{(s), 1}(n, x)=V^{(s)}(n, x) F_{s}^{(0)}(x), \quad V^{(s), 2}(n, x)=V^{(s)}(n, x)\left(1-F_{s}^{(0)}(x)\right) .
$$

Легко видеть, что из определения функции $F_{s}^{(0)}(x)$ вытекает равенство

$$
V^{(s), i}(n, x)=V^{(s), i}\left(-n, T^{n} x\right), \quad i=1,2 .
$$


Мы можем написать теперь следующее разложение для элемента

$$
\varphi^{(s)}(n, x)=d^{(s)}(x)+V_{R}^{(s), 1}(n, x)+U_{N R}^{(s)}(n, x)
$$

с $U_{N R}^{(s)}(n, x)=V_{R}^{(s), 2}(n, x)+V_{N R}^{(s)}(n, x)$. Легко проверить, что $V_{R}^{(s), 1}(n, x)$ равна нулю вне $\mathrm{P} 3 O_{t}^{(n)}$ и непрерьвна вместе со своими первой и второй производньмии в $\widetilde{O}_{t}^{(n)}$,

$$
\begin{gathered}
\left\|V_{R}^{(s), 1}(n, x)\right\|_{s}<\left\|V_{R}^{(s)}(n, x)\right\|_{s}+C \varepsilon_{s}, \quad\left\|U_{N R}^{(s)}(n, x)\right\|_{s}<C \varepsilon_{s}, \\
\left\|\frac{d}{d x} V_{R}^{(s), 1}(n, x)\right\|_{s}<\left\|\frac{d}{d x} V_{R}^{(s)}(n, x)\right\|_{s}+C \varepsilon_{s}^{3 / 4}, \\
\left\|\frac{d^{2}}{d x^{2}} V_{R}^{(s), 1}(n, x)\right\|_{s}<\left\|\frac{d^{2}}{d x^{2}} V_{R}^{(s)}(n, x)\right\|_{s}+C \varepsilon_{s}^{1 / 2}, \\
\left\|\frac{d U_{N R}^{(s)}(n, x)}{d x}\right\|_{s}<C \varepsilon_{s}^{3 / 4}, \quad\left\|\frac{d^{2}}{d x^{2}} U_{N R}^{(s)}(n, x)\right\|_{s}<C \varepsilon_{s}^{1 / 2} .
\end{gathered}
$$

Кроме того, $U_{N R}^{(s)}(n, x)$ равна нулю для $x \in O_{t}^{(n)} \backslash\left(\Delta_{t}^{(n), 1} \cup \Delta_{t}^{(n), 2}\right)$. Полученное разложение $\varphi^{(s)}(n, x)$ назовем сглаживанием типа 0 .

Мы разделяем все РЗ $O_{t}^{(n)}, \bar{s} \leqslant t \leqslant s$, на пять типов:

0) $O_{t}^{(n)}$ есть РЗ типа 0, если $s-t<g(s)$;

если $s-\bar{s}=g(s)$, то

1) $O_{\bar{s}}^{(n)}$ есть РЗ muпа 1, если $\sup _{x \in O_{\bar{s}}^{(n)}}\left|V_{R}^{(s), 1}(n, x)\right|<\frac{1}{4} \varepsilon_{s+1}$;

2) $O_{\bar{s}}^{(n)}$ есть Р3 muna 2 , если

2a) $\sup _{x \in O_{\bar{s}}^{(n)}}\left|V_{R}^{(s), 1}(n, x)\right| \geqslant \frac{1}{4} \varepsilon_{s+1}$,

$2 б)$ существует точка $x_{*} \in O_{\bar{s}}^{(n)}$ такая, что $d^{(s)}\left(x_{*}\right)=d^{(s)}\left(T^{n} x_{*}\right)$,

2в) $\left|V_{R}^{(s), 1}\left(n, x_{*}\right)\right|<\frac{1}{8} \varepsilon_{s+1}$;

3) $O_{\bar{s}}^{(n)}$ есть Р3 muпа 3 , если выполнены условия 2а) и 2б), а вместо 2в) выполняется условие $\left|V_{R}^{(s), 1}\left(n, x_{*}\right)\right| \geqslant \frac{1}{8} \varepsilon_{s+1} ;$

4) $O_{\bar{s}}^{(n)}$ есть Р3 muna 4 , если

$$
\sup _{x \in O \bar{s}}\left|V_{R}^{(s), 1}(n, x)\right| \geqslant \frac{1}{4} \varepsilon_{s+1}
$$

и для всех $x \in O_{\bar{s}}^{(n)}$ вьполняется неравенство $d^{(s)}(x) \neq d^{(s)}\left(T^{n} x\right)$.

Заметим, что согласно индуктивным предположениям может сушествовать не более одной точки $x_{*} \in O_{\bar{s}}^{(n)}$, для которой $d^{(s)}\left(x_{*}\right)=d^{(s)}\left(T^{n} x_{*}\right)$.

Итак, мы применили ко всем РЗ сглаживание типа 0. Этого сглаживания, как будет видно из дальнейшего изложения, достаточно для гладкой склейки преобразований нерезонансной части $U_{N R}^{(s)}(n, x)$ и резонансной части $V_{R}^{(s), 1}(n, x)$ в окрестности гранищ Р З. Однако для преобразования $V_{R}^{(s), 1}(n, x)$ внутри Р З необходимо определить 
дополнительное разложение $V_{R}^{(s), 1}(n, x)$ в зависимости от типа Р3, которое позволит получить функции, удовлетворяюшие индуктивным условиям гладкости на следуюшем шаге индукции.

Перейдем к рассмотрению РЗ типа 1.

ПРЕДЛОЖЕНИЕ 1. Для любой РЗ $O_{\bar{s}}^{(n)}$ muna 1

$$
\left\|V_{R}^{(s), 1}(n, x)\right\|_{s}<\varepsilon_{\bar{s}+1} .
$$

Более того, если

$$
\max _{x \in O_{\bar{s}}^{(n)}}\left|V_{R}^{(s), 1}(n, x)\right|<\varepsilon_{s+1}^{1 / 10},
$$

$m o\left\|V_{R}^{(s), 1}(n, x)\right\|_{s}<\varepsilon_{\bar{s}+1}$.

ДоказАтельСтво. Предположим противное, т.е. пусть $\left\|V_{R}^{(s), 1}(n, x)\right\|_{s} \geqslant \varepsilon_{\bar{s}+1}$. Тогда для некоторой точки $x \in O_{\bar{s}}^{(n)}$

$$
\left|V_{R}^{(s), 1}(n, x)\right| e^{\rho_{s}|n|} \geqslant \varepsilon_{\bar{s}+1}
$$

с $|n| \leqslant R_{\bar{s}}$. Таким образом,

$$
\varepsilon_{s+1}^{\frac{1}{10}} \geqslant \varepsilon_{\bar{s}+1} e^{-\rho|n|}
$$

или

$$
\left.\varepsilon^{\frac{1}{\bar{s}}(1+1}+\kappa\right)^{s-\bar{s}} \geqslant e^{-\rho|n|} \geqslant e^{-\rho R_{\bar{s}}}
$$

Следовательно,

$$
\rho R_{\bar{s}}>(1+\kappa)\left(\frac{1}{10}(1+\kappa)^{s-\bar{s}}-1\right) \ln \varepsilon_{\bar{s}}^{-1}
$$

или

$$
\rho \Gamma(\bar{s})^{2}>\left(\frac{1}{10}(1+\kappa)^{s-\bar{s}}-1\right)(1+\kappa), \quad \rho \Gamma(\bar{s})^{2}>\Gamma \rho s^{2}-1-\kappa .
$$

Так как $\bar{s}<s$, то последнее неравенство невозможно при $\rho \Gamma>1+\kappa=\frac{5}{4}$.

Это предложение показьвает, что рассмотрение РЗ $O_{\bar{s}}^{(n)}$ типа 1 может быть сведено к рассмотрению РЗ порядка $\bar{s}+1$. Чтобы сделать это, определим для каждой $\mathrm{P} 3 O_{\bar{s}}^{(n)}$ типа 1 функцию $F_{n, \bar{s}}^{(1)}(x)$ следуюшим образом. Рассмотрим РЗ

$$
O_{\bar{s}+1}^{(n)} \subset O_{\bar{s}}^{(n)}: O_{\bar{s}+1}^{(n)}=\left\{x:\left|d^{(\bar{s}+1)}(x)-d^{(\bar{s}+1)}\left(T^{n} x\right)\right|<\delta_{\bar{s}+1}\right\} \cap O_{\bar{s}}^{(n)} .
$$

Пусть $O_{\bar{s}}^{(n)}=\Delta_{n, \bar{s}}=\left(x_{1}, x_{2}\right), O_{\bar{s}+1}^{(n)}=\Delta_{n, \bar{s}+1}=\left(x_{1}^{\prime}, x_{2}^{\prime}\right), x_{1}<x_{1}^{\prime}<x_{2}^{\prime}<x_{2}$. Ясно, что $\left|\Delta_{n, \bar{s}+1}\right|>C \delta_{\bar{s}+1}$. Положим $\Delta_{\bar{s}}^{(n), 1}=\left(x_{1}^{\prime}, x_{1}^{\prime \prime}\right), \Delta_{\bar{s}}^{(n), 2}=\left(x_{2}^{\prime \prime}, x_{2}^{\prime}\right)$, где $\left|\Delta_{\bar{s}}^{(n), 1}\right|=\left|\Delta_{\bar{s}}^{(n), 2}\right|=\varepsilon_{\bar{s}+1}^{1 / 4}$. Определим $F_{n, \bar{s}}^{(1)}(x)=0$ вне $O_{\bar{s}+1}^{(n)}, F_{n, \bar{s}}^{(1)}(x)=1$ для $x \in\left(x_{1}^{\prime}, x_{2}^{\prime \prime}\right), F_{n, \bar{s}}^{(1)}(x)=F_{\Delta_{\bar{s}}^{(n), 2}}(x)$ для $x \in \Delta_{\bar{s}}^{(n), 1}$ и $F_{n, \bar{s}}^{(1)}(x)=1-F_{\Delta_{\bar{s}}^{(n), 1}}(x)$ для $x \in \Delta_{\bar{s}}^{(n), 2}$, где $F_{\Delta}(x)$ - введенная выше вспомогательная функция для отрезка $\Delta$. 
Наконец, положим $F_{\bar{s}}^{(1)}(x)=\sum F_{n, \bar{s}}^{(1)}(x)$, где суммирование проводится по всем РЗ типа 1. Функция $F_{s}^{(1)}(x)$ корректно определена в силу условия $\left.2_{s}\right)$.

Представим $V_{R}^{(s), 1}(n, x)$ в виде суммы двух слагаемых:

$$
U_{R}^{(s), 1}(n, x)=V_{R}^{(s), 1}(n, x) F_{s}^{(1)}(x) \quad \text { и } U_{R}^{(s), 2}(n, x)=V_{R}^{(s), 1}(n, x)-U_{R}^{(s), 1}(n, x) .
$$

Ясно, что $U_{R}^{(s), 1}(n, x) \equiv 0$ вне объединения всех Р3 типа $1, U_{R}^{(s), 2}(n, x) \equiv 0$ вне

$$
\bigcup\left[\left(O_{\bar{s}}^{(n)}-\left(O_{\bar{s}+1}^{(n)}\right)\right) \cup\left(\Delta_{\bar{s}}^{(n), 1} \cup \Delta_{\bar{s}}^{(n), 1}\right)\right],
$$

где внешнее объединение производится по всем Р3 типа 1. Для того, чтобы оценить производные функций $U_{R}^{(s), 1}(n, x)$ и $U_{R}^{(s), 2}(n, x)$, нам потребуется следующее утверждение.

ПРЕДЛОЖЕНИЕ 2. Для РЗ $O_{\bar{s}}^{(n)}$ muna 1

$$
\max _{x \in O_{\bar{s}}^{(n)}}\left|\frac{d V_{R}^{(s), 1}(n, x)}{d x}\right|<\varepsilon_{s+1}^{1 / 2} .
$$

ДокАЗАТЕЛЬСТвО. Пусть $\tau_{1}\left(O_{\bar{s}}^{(n)}\right)$ - момент возникновения Р $3 O_{\bar{s}}^{(n)}$, определяемьй как $\min _{x \in O_{\bar{s}}^{(n)}} k(x)$, где $k(x)$ - минимальное значение $l$, для которого $\tau^{(l)}(x)=l$, $\tau^{(l-1)}(x) \neq l-1, \tau^{(p)}(x)=p$ для $l<p \leqslant \bar{s}$. Легко показать, что $k(x)$ не зависит от $x$. Из индуктивных предположений следует, что

$$
\left\|V_{R}^{(s), 1}(n, x)\right\|_{s}<C \varepsilon_{\tau_{1}},\left\|\frac{d V_{R}^{(s), 1}(n, x)}{d x}\right\|_{s}<C \varepsilon_{\tau_{1}}^{3 / 4},\left\|\frac{d^{2} V_{R}^{(s), 1}(n, x)}{d^{2} x}\right\|_{s}<C \varepsilon_{\tau_{1}}^{1 / 2} .
$$

Предположим, что в некоторой точке $\bar{x} \in O_{\bar{s}}^{(n)}$ вьполнено неравенство

$$
\left|\frac{d V_{R}^{(s), 1}(n, \bar{x})}{d x}\right| \geqslant \varepsilon_{s+1}^{1 / 2} .
$$

Тогда

$$
\left|\frac{d V_{R}^{(s), 1}(n, x)}{d x}\right| \geqslant \frac{1}{2} \varepsilon_{s+1}^{1 / 2}
$$

для любой точки $x$ такой, что $|x-\bar{x}| \leqslant \varepsilon_{s+1}^{1 / 2} \varepsilon_{\tau_{1}}^{-1 / 4}$. В самом деле,

$$
\begin{aligned}
\left|\frac{d V_{R}^{(s), 1}(n, x)}{d x}\right| & >\left|\frac{d V_{R}^{(s), 1}(n, \bar{x})}{d x}\right|-\left|\frac{d^{2} V_{R}^{(s), 1}(n, \widetilde{x})}{d x^{2}}\right| x-\bar{x} \mid \\
& >\left|\frac{d V_{R}^{(s), 1}(n, \bar{x})}{d x}\right|-C \varepsilon_{\tau_{1}}^{1 / 2}|x-\widetilde{x}| \geqslant \frac{1}{2} \varepsilon_{s+1}^{1 / 2},
\end{aligned}
$$


где $\widetilde{x}=\bar{x}+\Theta(x-\bar{x}), 0<\Theta<1$. Следовательно,

$$
\left|V_{R}^{(s), 1}(n, x)-V_{R}^{(s), 1}(n, \bar{x})\right|>\frac{1}{2} \varepsilon_{s+1}^{1 / 2}|x-\bar{x}| .
$$

Для $x_{1}^{ \pm}=\bar{x} \pm \varepsilon_{s+1}^{1 / 2} \varepsilon_{\tau_{1}}^{-1 / 4}$ получаем, что

$$
\left|V_{R}^{(s), 1}\left(n, x^{ \pm}\right)-V^{(s), 1}(n, \bar{x})\right|>2 \varepsilon_{s+1} \quad \text { и }\left|V_{R}^{(s), 1}\left(n, x^{ \pm}\right)\right|>\varepsilon_{s+1} .
$$

По крайней мере одна из точек $x^{+}, x^{-}$принадлежит $O_{\bar{s}}^{(n)}$, что противоречит определению РЗ типа 1.

Из предложений 1 и 2 легко следует, что

$$
\left\|U_{k}^{(s), 1}(n, x)\right\|_{s}<\varepsilon_{\bar{s}+1}, \quad\left\|\frac{d U_{R}^{(s), 1}(n, x)}{d x}\right\|_{s}<C \varepsilon_{\tau_{1}}^{3 / 4}, \quad\left\|\frac{d^{2} U_{R}^{(s), 1}(n, x)}{d x^{2}}\right\|_{s}<C \varepsilon_{\tau_{1}}^{1 / 2}
$$

$$
\left|U_{R}^{(s), 2}(n, x)\right|<\varepsilon_{s+1}, \quad\left|\frac{d U_{R}^{(s), 2}(n, x)}{d x}\right|<\varepsilon_{\bar{s}+1}^{1 / 2}, \quad\left|\frac{d^{2} U_{R}^{(s), 2}(n, x)}{d x^{2}}\right|<C \varepsilon_{\tau_{1}}^{1 / 2} .
$$

Теперь рассмотрим РЗ типа 2. Для каждой Р3 $O_{\bar{s}}^{(n)}$ типа 2 построим разложение $V_{R}^{(s), 1}(n, x)=U_{R}^{(s), 1}(n, x)+U_{R}^{(s), 2}(n, x)$. Если $\left\|V_{R}^{(s), 1}(n, x)\right\|_{s}<\varepsilon \bar{s}+1$, то это разложение строится точно так же, как и для РЗ типа 1.

Пусть $\left\|V_{R}^{(s), 1}(n, x)\right\|_{s} \geqslant \varepsilon_{\bar{s}+1}$ и $d^{(s)}\left(x_{*}\right)=d^{(s)}\left(T^{n} x_{*}\right)$. Тогда существует ближайшая к $x_{*}$ точка $x^{*}$ такая, что $\left|V_{R}^{(s), 1}\left(n, x^{*}\right)\right|=\frac{1}{4} \varepsilon_{s+1}$. Кроме того, из определения РЗ типа 2 следует, что сушествует такая $x^{* *}$, лежашая между $x_{*}$ и $x^{*}$, что $\left|V_{R}^{(s), 1}\left(n, x^{* *}\right)\right|=\frac{1}{8} \varepsilon_{\bar{s}+1}$. Из условия $\left\|V_{R}^{(s), 1}(n, x)\right\|_{s}>\varepsilon_{\bar{s}+1}$ и предложения 1 вытекает сушествование такой точки $\bar{x}$, ближайшей к $x^{*}$, что $\left|V_{R}^{(s), 1}(n, \bar{x})\right|=\varepsilon_{s+1}^{1 / 2}$ и $x^{*}$ лежит между $x^{* *}$ и $\bar{x}$. Можно считать, что $x_{*}<x^{* *}<x^{*}<\bar{x}$. (Случай $\bar{x}<x^{*}<x^{* *}<x_{*}$ рассматривается аналогично.)

ПРЕДЛОЖЕНИЕ 3. На отрезКе $\left[x_{*}, \bar{x}\right]$

$$
\left|\frac{d V_{R}^{(s), 1}(n, x)}{d x}\right|<\varepsilon_{\bar{s}+1}^{1 / 4} .
$$

ДокАЗАТЕЛЬство. Прежде всего заметим, что длина отрезка $\left[x_{*}, \bar{x}\right]$ больше

$$
\frac{\left|\varepsilon_{s+1}^{1 / 2}-\varepsilon_{s+1}\right|}{\max _{x \in\left[x_{*}, \bar{x}\right]}\left|\frac{d}{d x} V_{R}^{(s), 1}(n, x)\right|} \geqslant C\left|\varepsilon_{s+1}^{1 / 2}-\varepsilon_{\bar{s}+1}\right| \varepsilon_{\tau_{1}}^{-3 / 4}
$$

Предположим, что $\widetilde{x} \in\left[x_{*}, \bar{x}\right]$ и $\left|\frac{d}{d x} V_{R}^{(s), 1}(n, \widetilde{x})\right|>\varepsilon_{s+1}^{1 / 2}$. Тогда для всех $x \in\left[x_{*}, \bar{x}\right]$ таких, что $|\widetilde{x}-x| \leqslant \varepsilon_{s+1}^{1 / 2} \varepsilon_{\tau_{1}}^{-1 / 3}$, выполнено неравенство

$$
\left|\frac{d}{d x} V^{(s), 1}(n, x)\right|>\frac{1}{2} \varepsilon_{s+1}^{1 / 2} .
$$


В самом деле,

$$
\left|\frac{d}{d x} V^{(s), 1}(n, x)\right|>\left|\frac{d}{d x} V^{(s), 1}(n, \widetilde{x})\right|-C \varepsilon_{\tau_{1}}^{1 / 2}|x-\widetilde{x}|>\frac{1}{2} \varepsilon_{s+1}^{1 / 2} .
$$

Но тогда для $x \in\left[x_{*}, \bar{x}\right]$ таких, что

$$
|\widetilde{x}-x|=\varepsilon_{s+1}^{1 / 2} \varepsilon_{\tau_{1}}^{-1 / 3},
$$

модуль разности

$$
\left|V_{R}^{(s), 1}(n, x)-V_{R}^{(s), 1}(n, \widetilde{x})\right|>\frac{1}{2} \varepsilon_{s+1}^{1 / 2} \varepsilon_{s+1}^{1 / 2} \varepsilon_{\tau_{1}}^{-1 / 3}>2 \varepsilon_{s+1}^{1 / 2} .
$$

Следовательно, $\left|V_{R}^{(s), 1}(n, x)\right|>\varepsilon_{s+1}^{1 / 2}$, что противоречит предположению о том, что $\bar{x}$ является ближайшей к $x_{*}$ точкой, в которой $\left|V_{R}^{(s), 1}(n, x)\right|=\varepsilon_{s+1}^{1 / 2}$.

Пусть $\Delta_{\bar{s}}^{(n), 2}=\left[x^{*}, x_{1}^{*}\right]$ есть отрезок длины $\varepsilon_{s+1}^{1 / 2} \varepsilon_{\tau_{1}}^{-1 / 4}$. Мы определяем $U_{R}^{(s), 1}(n, x)$ для $x \in O_{\bar{s}}^{(n)}$ и $x>x_{*}$ следующим образом:

$$
\begin{array}{r}
U_{R}^{(s), 1}(n, x)=\left[V_{R}^{(s), 1}\left(n, x^{*}\right)-\frac{d V_{R}^{(s), 1}\left(n, x^{*}\right)}{d x}\left(x-x^{*}\right)+\frac{1}{2} \frac{d^{2} V_{R}^{(s), 1}\left(n, x^{*}\right)}{d x^{2}}\left(x-x^{*}\right)^{2}\right] \\
\times\left(1-F_{\Delta_{\bar{s}}^{(n), 2}(x)}\right)
\end{array}
$$

для $x \in \Delta_{\bar{s}}^{(n), 2}$,

$$
U_{R}^{(s), 1}(n, x)=V_{R}^{(s), 1}(n, x)
$$

для $x \in\left[x_{*}, x^{*}\right]$,

$$
U_{R}^{(s), 1}(n, x)=0
$$

для $x \geqslant x_{1}^{*}$.

Если $\bar{x}^{*} \geqslant x_{*}$ есть ближайшая к $x_{*}$ точка, в которой $V_{R}^{(s), 1}\left(n, \widetilde{x}^{*}\right)=\frac{1}{4} \varepsilon_{s+1}$, то мы аналогичным образом выбираем отрезок $\Delta_{\bar{s}}^{(n), 1}=\left[\bar{x}_{1}^{*}, \bar{x}^{*}\right]$ и полагаем

$$
\begin{aligned}
U_{R}^{(s), 1}(n, x)=\left[V_{R}^{(s), 1}\left(n, \bar{x}^{*}\right)-\frac{d V_{R}^{(s), 1}\left(n, \bar{x}^{*}\right)}{d x}\left(x-\bar{x}^{*}\right)+\frac{1}{2} \frac{d^{2} V_{R}^{(s), 1}(n, x)}{d x^{2}}\left(x-\bar{x}^{*}\right)^{2}\right] \\
\times F_{\Delta_{\bar{s}}^{(n), 1}(x)}
\end{aligned}
$$

для $x \in \Delta_{\bar{s}}^{(n), 1}$,

$$
U_{R}^{(s), 1}(n, x)=V_{R}^{(s), 1}(n, x)
$$

для $x<\bar{x}_{1}^{*}$ и

$$
U_{R}^{(s), 1}(n, x)=0
$$

для $x<x_{1}^{*}$.

Если же такой точки $\bar{x}^{*}$ не сушествует, то мы строим $U_{R}^{(s), 1}(n, x)$ для $x<x_{*}$ таким же образом, как и для РЗ типа 1. 
Из предложения 3 следует справедливость следующих оценок:

$$
\left\|U_{R}^{(s), 1}(n, x)\right\|_{s}<\varepsilon_{\bar{s}+1}, \quad\left\|U_{R}^{(s), 1}(n, x)\right\|_{s}<C \varepsilon_{\tau_{1}}^{3 / 4}, \quad\left\|\frac{d^{2} U_{R}^{(s), 1}(n, x)}{d x^{2}}\right\|_{s}<C \varepsilon_{\tau_{1}}^{1 / 2}
$$

и

$$
\begin{gathered}
\left|V_{R}^{(s), 1}(n, x)\right|<C \varepsilon_{s+1} \varepsilon_{\tau_{1}}^{-1 / 4}, \\
\left|\frac{d U_{R}^{(s), 1}(n, x)}{d x}\right|<C \varepsilon_{s+1} \varepsilon_{\tau_{1}}^{-1 / 2}, \quad\left|\frac{d^{2} U_{R}^{(s), 1}(n, x)}{d x^{2}}\right|_{s}<C \varepsilon_{\tau_{1}}^{1 / 2} .
\end{gathered}
$$

Для РЗ типа 0,3 и 4 полагаем $U_{R}^{(s), 1}(n, x) \equiv 0$.

Таким образом, мы построили новое разложение элемента $\varphi^{(s)}(n, x)$ :

$$
\varphi^{(s)}(x)=d^{(s)}(x)+U_{R}^{(s), 1}(n, x)+U_{R}^{(s), 2}(n, x)+U_{N R}^{(s)}(n, x),
$$

где $U_{R}^{(s), 2}(n, x)=V_{R}^{(s), 1}(n, x)-U_{R}^{(s), 1}(n, x)$.

ЗАмечАнИЕ. Из предложения 3 вытекает, что длина отрезка $\left[x_{*}, x^{*}\right]$ больше $C \varepsilon_{s+1}^{1 / 2} \varepsilon_{\tau_{1}}^{-1 / 2}$ и, следовательно,

$$
\left|d^{(s)}\left(x^{*}\right)-d^{(s)}\left(T^{n} x^{*}\right)\right|>C_{1} \varepsilon_{s+1}^{1 / 2} \varepsilon_{\tau_{1}}^{-1 / 2} .
$$

3.2. Преобразование нерезонансной части. Итак, в конце предыдущего раздела мы получили представление

$$
\varphi^{(s)}(n, x)=d^{(s)}(n, x)+V_{R}^{(s), 1}(n, x)+U_{N R}^{(s)}(n, x),
$$

причем в этом разделе нам достаточно иметь только следующие оценки:

$\left\|U_{N R}^{(s)}(n, x)\right\|_{s}<\varepsilon_{s},\left\|\frac{d U_{N R}^{(s)}(n, x)}{d x}\right\|_{s}<\varepsilon_{s}^{3 / 4},\left\|\frac{d^{2} U_{R}^{(s)}(n, x)}{d x^{2}}\right\|_{s}<\varepsilon_{s}^{1 / 2}, U_{N R}^{(s)}(n, x)=0$ для всех пар $(n, x)$ таких, что $\left|d^{(s)}(x)-d^{(s)}\left(T^{n} x\right)\right|<\frac{1}{2} \delta_{s},\left\|V_{R}^{(s), 1}(n, x)\right\|_{s}<\varepsilon_{\bar{s}}$, производные $\frac{d V_{R}^{(s), 1}(n, x)}{d x}$ и $\frac{d^{2} V_{R}^{(s), 1}(n, x)}{d x^{2}}$ ограничены по модулю некоторой абсолютной постоянной,

$$
\left|d^{(s)}(x)\right|<C, \quad\left|\frac{d}{d x}\left(d^{(s)}(x)\right)\right|<C \text { и }\left|\frac{d^{2}}{d x^{2}}\left(d^{(s)}(x)\right)\right|<\frac{C}{\varepsilon_{s-1}} .
$$

Представим $U_{N R}^{(s)}(n, x)$ в виде двух слагаемых:

$$
U_{N R}^{(s)}(n, x)=U_{N R}^{(s), 1}(n, x)+U_{N R}^{(s), 2}(n, x),
$$

где $U_{N R}^{(s), 1}(n, x)=U_{N R}^{(s)}(n, x)$ для $|n| \leqslant R_{s}, U_{R}^{(s), 1}(n, x)=0$ для $|n|>R_{s}, U_{N R}^{(s), 2}(n, x)=$ $U_{N R}^{(s)}(n, x)-U_{N R}^{(s), 1}(n, x)$. 
ПРЕДЛОЖЕНИЕ 4. Справедливы следующие оценки:

$$
\begin{gathered}
\left\|U_{N R}^{(s), 2}(n, x)\right\|_{s+1} \leqslant \varepsilon_{s}^{\frac{3}{\pi^{2}} \rho \Gamma}\left\|U_{N R}^{(s)}(n, x)\right\|_{s}, \\
\left\|\frac{d U_{N R}^{(s), 2}(n, x)}{d x}\right\|_{s+1} \leqslant \varepsilon_{s}^{\frac{3}{\pi^{2}} \rho \Gamma}\left\|\frac{d U_{N R}^{(s)}(n, x)}{d x}\right\|_{s} \\
\left\|\frac{d^{2} U_{R}^{(s), 2}(n, x)}{d x^{2}}\right\|_{s+1} \leqslant \varepsilon_{s}^{\frac{3}{\pi^{2}} \rho \Gamma}\left\|\frac{d^{2} U_{R}^{(s)}(n, x)}{d x^{2}}\right\|_{s} .
\end{gathered}
$$

ДОКАЗАТЕЛЬСТВо.

$$
\begin{gathered}
\left\|U_{N R}^{(s), 2}(n, x)\right\|_{s+1}=\sum_{|n|>R_{s}} \operatorname{ess} \sup \left|U_{N R}^{(s)}(n, x)\right| e^{\rho_{s+1}|n|} \leqslant e^{-\left(\rho_{s}-\rho_{s+1}\right) R_{s}}, \\
\sum_{|n|>R_{s}} \operatorname{ess} \sup \left|U_{N R}^{(s)}(n, x)\right| e^{\rho_{s}|n|} \leqslant \varepsilon_{s}^{\frac{3}{\pi^{2}} \rho \Gamma}\left\|U_{N R}^{(s)}\right\|_{s} .
\end{gathered}
$$

Аналогично получаются оценки производных.

Для упрощения формул введем некоторые обозначения. Для любого обратимого элемента $a \in A^{(\rho)}$ обозначим $\operatorname{Ad}_{a}$ отображение $A^{(\rho)} \rightarrow A^{(\rho)}: \operatorname{Ad}_{a}(\varphi)=a^{-1} \varphi$ для любого $\varphi \in A^{(\rho)}$ и через $\operatorname{ad}_{b}$ отображение $A^{(\rho)} \rightarrow A^{(\rho)}: \operatorname{ad}_{b}(\varphi)=[\varphi, b]=\varphi b-b \varphi$ для любого $\varphi \in A^{(\rho)}$ и произвольного фиксированного $b \in A^{(\rho)}$. Ясно, что если $a=\exp \{b\}$, то $a-$ обратимьй элемент алгебры $A^{(\rho)}$ и $a^{-1}=\exp \{-b\}$.

Далее мы применяем стандартньй метод КАМ-теории, используя хорошо известную формулу

$$
\operatorname{Ad}_{a}(\varphi)=\varphi+\sum_{k=1}^{\infty} \frac{\operatorname{ad}_{b}\left(\operatorname{ad}_{b}\left(\ldots \operatorname{ad}_{b}(\varphi)\right) \ldots\right)}{k !}
$$

где $a=\exp (b)$.

ПРЕДЛОЖЕНИЕ 5. Существует такой унитарный әлемент $u^{(s)}=u^{(s)}(n, x) \in$ $A^{\left(\rho_{s}\right)}$, чmo

$$
\operatorname{Ad}_{u^{(s)}}\left(\varphi^{(s)}\right)=\widetilde{d}^{(s)}(x)+\widetilde{V}_{R}^{(s), 1}(n, x)+\widetilde{U}_{N R}^{(s), 1}(n, x),
$$

причем справедливы следующие оценки:

$$
\begin{gathered}
\left|\widetilde{d}^{(s)}(x)-d^{(s)}(x)\right|<\varepsilon_{s+1}, \quad\left|\frac{d}{d x}\left(\widetilde{d}^{(s)}(x)-d^{(s)}(x)\right)\right|<\varepsilon_{s}^{1-4 \mu} \varepsilon_{s}^{3 / 4} \\
\left|\frac{d^{2}}{d x^{2}}\left(\widetilde{d}^{(s)}(x)-d^{(s)}(x)\right)\right|<\varepsilon_{s}^{1-8 \mu} \varepsilon_{s}^{1 / 2}, \quad \widetilde{V}_{R}^{(s), 1}(n, x)=0
\end{gathered}
$$

вне $P 3 O_{t}^{(n)}, \bar{s} \leqslant t \leqslant s$,

$$
\begin{gathered}
\left\|\widetilde{V}_{R}^{(s), 1}(n, x)-V_{R}^{(s), 1}(n, x)\right\|_{s+1}<\left\|V_{R}^{(s), 1}(n, x)\right\|_{s} \varepsilon_{s}^{1-2 \mu}, \\
\left\|\frac{d}{d x}\left(\widetilde{V}_{R}^{(s), 1}(n, x)-V_{R}^{(s), 1}(n, x)\right)\right\|_{s+1}<\left\|\frac{d}{d x} V_{R}^{(s), 1}(n, x)\right\| \varepsilon_{s}^{1-2 \mu} \\
\left\|\frac{d^{2}}{d x^{2}}\left(\widetilde{V}_{R}^{(s), 1}(n, x)-V_{R}^{(s), 1}(n, x)\right)\right\|_{s+1}<\left\|\frac{d^{2}}{d x^{2}} V_{R}^{(s), 1}(n, x)\right\| \varepsilon_{s}^{1-2 \mu} .
\end{gathered}
$$


Далее, $\widetilde{U}_{N R}^{(s)}(n, x)=0$ для всех nар $(n, x)$, для которых

$$
\begin{gathered}
\left|\widetilde{d}^{(s)}(x)-\widetilde{d}^{(s)}\left(T^{n} x\right)\right|<\delta_{s}-C \varepsilon_{s} \varepsilon \bar{s}, \quad\left\|\widetilde{U}_{N R}^{(s)}(n, x)\right\|_{s+1}<\varepsilon_{s+1}^{6}, \\
\left\|\frac{d}{d x} \widetilde{U}_{N R}^{(s), 1}(n, x)\right\|_{s+1}<\varepsilon_{s+1}^{5}, \quad\left\|\frac{d^{2}}{d x^{2}} \widetilde{U}_{N R}^{(s), 1}(n, x)\right\|_{s+1}<\varepsilon_{s+1}^{4}
\end{gathered}
$$

Для унитарного әлемента $u^{(s)}(n, x)$ справедливы следующие неравенства:

$$
\begin{gathered}
\left\|u^{(s)}(n, x)-\delta_{0 n}\right\|_{s}<C \varepsilon_{s} \varepsilon_{\bar{s}}^{-\mu}, \\
\left\|\frac{d}{d x} u^{(s)}(n, x)\right\|_{s}<C \varepsilon_{s}^{3 / 4}, \quad\left\|\frac{d^{2}}{d x^{2}} u^{(s)}(n, x)\right\|_{s}<\varepsilon_{s-1}^{1 / 8} .
\end{gathered}
$$

ДокАЗАТЕльство. Для любого элемента $a=a(n, x) \in A^{\left(\rho_{s}\right)}$ определим элемент $a^{(t r)}=a^{(t r)}(n, x)$, полагая $a^{(t r)}(n, x)=a(n, x)$ для $|n| \leqslant R_{s}$ и $a^{(t r)}(n, x)=0$ для $|n|>R_{s}$. Будем искать $u^{(s)}$ в виде $\prod_{i=1}^{M(s)} u^{(s), i}$, где $u^{(s), i}-$ унитарные элементы алгебры $A^{\left(\rho_{s}\right)}$, а $M(s)$ будет указано в ходе доказательства.

Сначала найдем элемент $u^{(s), 1}$, которьй мы будем искать в виде

$$
u^{(s), 1}=\exp \left\{i w^{(s), 1}\right\}
$$

где $w^{(s), 1}$ - некоторьй самосопряженный элемент алгебры $A^{\left(\rho_{s}\right)}$. С целью унификации обозначений положим $\varphi^{(s)}=\varphi^{(s), 0}$. Напишем формулу $(*) \mathrm{c} \varphi=\varphi^{(s), 0}, a=u^{(s), 1}$. Мы получим:

$$
\begin{aligned}
\operatorname{Ad}_{u^{(s), 1}}\left(\varphi^{(s), 0}\right)=d^{(s)}(x)+ & \operatorname{Ad}_{u^{(s), 1}}\left(V_{R}^{(s), 1}\right)+U_{N R}^{(s), 1}+\operatorname{ad}_{i w^{(s), 1}}\left(d^{(s)}(x)\right)+\operatorname{ad}_{i w^{(s), 1}}\left(U_{N R}^{(s), 1}\right) \\
& +\sum_{k=2}^{\infty} \frac{\operatorname{ad}_{i w^{(s), 1}}\left(\operatorname { a d } _ { i w ^ { ( s ) , 1 } } \left(\ldots\left(\operatorname{ad}_{i w^{(s), 1}}\left(d^{(s)}(x)+U_{N R}^{(s), 1}\right) \ldots\right)\right.\right.}{k !}
\end{aligned}
$$

Полагая $w^{(s), 1}(n, x)=\frac{i U_{N R}^{(s), 1}(n, x)}{d^{(s)}(x)-d^{(s)}\left(T^{n} x\right)}$ для таких $(n, x)$, для которых $U_{N R}^{(s), 1}(n, x) \neq 0$, и $w^{(s), 1}(n, x)=0$, если $U_{N R}^{(s), 1}(n, x)=0$, находим, что $w^{(s), 1}$ есть решение уравнения

$$
U_{N R}^{(s), 1}+\operatorname{ad}_{i w^{(s), 1}}\left(d^{(s)}(x)\right)=0
$$

Легко проверить, что

$$
\begin{gathered}
\left\|w^{(s), 1}\right\|_{s}<\frac{2 \varepsilon_{s}}{\varepsilon \frac{\mu}{s}} \\
\left\|\operatorname{Ad}_{u^{(s), 1}}\left(V_{N R}^{(s), 1}\right)\right\|_{s}<2 \varepsilon_{s}^{2} \varepsilon_{\bar{s}}^{-\mu} \text { и }\left\|\operatorname{Ad}_{u^{(s), 1}}\left(V_{R}^{(s), 1}\right)-V_{R}^{(s), 1}\right\|_{s}<\varepsilon \varepsilon_{\bar{s}}^{1-2 \mu} \varepsilon_{s} .
\end{gathered}
$$

Более того, представим элемент $\varphi^{(s), 1}=\operatorname{Ad}_{u^{(s), 1}}\left(\varphi^{(s), 0}\right)$ в виде суммы

$$
d_{1}^{(s)}(x)+V_{R}^{(s), 1}(n, x)+V_{R, 1}^{(s), 1}(n, x)+U_{N R, 1}^{(s)}(n, x),
$$


где $d_{1}^{(s)}(x)=\varphi^{(s), 1}(0, x)$

$$
U_{N R, 1}^{(s)}(n, x)=\left[\operatorname{Ad}_{u^{(s), 1}}\left(U_{N R}^{(s), 1}\right)\right](n, x)+\left[\operatorname{Ad}_{u^{(s), 1}}\left(V_{R}^{(s)}\right)\right](n, x)-V_{R}^{(s), 1}(n, x)
$$

для $(n, x)$, не являюшихся $t$-резонансными для $\bar{s} \leqslant t \leqslant s, U_{N R, 1}^{(s)}(n, x)=0$ для остальных пар $(n, x)$ и

$$
V_{R, 1}^{(s), 1}(n, x)=\operatorname{Ad}_{u^{(s), 1}}\left(\varphi^{(s), 0}\right)(n, x)-V_{R}^{(s), 1}(n, x)-U_{N R, 1}^{(s)}(n, x) .
$$

Применим к $\varphi^{(s), 1}$ сглаживание типа 0 , описанное в разделе 3.1 , и запишем следующее разложение:

$$
\varphi^{(s), 1}(n, x)=d_{1}^{(s)}(x)+\bar{V}_{R, 1}^{(s), 1}(n, x)+\bar{U}_{N R, 1}^{(s)}(n, x),
$$

где

$$
\bar{V}_{R, 1}^{(s), 1}(n, x)=V_{R}^{(s), 1}(n, x)+\overline{\bar{V}}_{R}^{(s), 1}(n, x)
$$

и $\overline{\bar{V}}_{R}^{(s), 1}(n, x)$ равно нулю вне РЗ $O_{t}^{(n)}, \bar{s} \leqslant t \leqslant s$. Отметим, что

$$
\left\|\overline{\bar{V}}_{R}^{(s), 1}\right\|_{s}<C \frac{\varepsilon_{s}^{2}}{\varepsilon_{\bar{s}}^{\mu}}
$$

Последнее неравенство вытекает из того, что $\operatorname{ad}_{i w}(s), 1\left(V_{R}^{(s), 1}(n, x)\right)=0$ для всех пар $(n, x)$ таких, что $x \in O_{t}^{(n)}, \bar{s} \leqslant t \leqslant s$, и разложение для $\operatorname{Ad}_{u^{(s), 1}}\left(V_{R}^{(s), 1}\right)$ начинается только с членов второго порядка по $w^{(s), 1}$. Для того, чтобы убедиться, что $\operatorname{ad}_{i w(s), 1}\left(V_{R}^{(s), 1}\right)$ обладает указанным свойством, запишем явное выражение для $\operatorname{ad}_{i w^{(s), 1}}\left(V_{R}^{(s), 1}\right)$ :

$$
\begin{aligned}
& \quad\left[\operatorname{ad}_{i w^{(s), 1}}\left(V_{R}^{(s), 1}\right)\right](n, x) \\
& \quad=i \sum_{|m| \leqslant R_{s}}\left\{V_{R}^{(s), 1}\left(n-m, T^{m} x\right) w^{(s), 1}(m, x)-w^{(s), 1}\left(n-m, T^{m} x\right) V_{R}^{(s), 1}(m, x)\right\} .
\end{aligned}
$$

Если $(n, x)$ - такая пара, что $x \in O_{t}^{(n)}$ для некоторого $\bar{s} \leqslant t \leqslant s$, то пары $(m, x)$ не являются $t_{1}$-резонансными ни при каком $t_{1}, \bar{s} \leqslant t_{1} \leqslant s$, так как в противном случае было бы непусто $O_{t_{1}}^{(m)} \cap O_{t}^{(n)}$, что невозможно в силу индуктивного предположения $\left.2_{s}\right)$. Следовательно, $V_{R}^{(s), 1}(m, x)=0$ при $n \neq m$, а при $n=m$ вьполняется $w^{(s), 1}\left(n-m, T^{m} x\right)=w^{(s), 1}\left(0, T^{m} x\right)=0$. С другой стороны, вместе с парой $(n, x)$ и пара $\left(-n, T^{n} x\right)$ является $t$-резонансной, а значит, $V_{R}^{(s), 1}\left(n-m, T^{m} x\right)=$ $V_{R}^{(s), 1}\left(m-n, T^{n} x\right)=0$ для $m-n \neq-n$, т.е. $m \neq 0 ; w^{(s), 1}(m, x)=0$ при $m=0$. Поэтому $\left[\operatorname{ad}_{i w^{(s), 1}}\left(V_{R}^{(s), 1}\right)\right](n, x)=0$ для указанных пар $(n, x)$.

Мы получили элемент $\varphi_{1}^{(s)}$, резонансная часть которого имеет меньший порядок. Повторяя эту процедуру $M(s)$ раз, где $M(s)=6\left[(1+\kappa)^{s-\bar{s}}(1-2 \mu)^{-1}\right]+1$, мы построим элементы $\varphi^{(s), 2}, \varphi^{(s), 3}, \ldots, \varphi^{(s), M(s)}$ и унитарные элементы $u^{(s), 1}, \ldots, u^{(s), M(s)}$ такие, что элементы $\widetilde{\varphi}^{(s)}=\varphi^{(s), M(s)}=\operatorname{Ad}_{u^{(s)}} \varphi^{(s)}$ и $u^{(s)}=\prod_{i=1}^{M(s)} u^{(s), i}$ будут удовлетворять предложению 5. 
3.3. Преобразование резонансной части. Частичное и полное расщепление резонансов. В предыдущем разделе мы построили унитарньй элемент $u^{(s)} \in A^{\left(\rho_{s}\right)}$ такой, что элемент

$$
\widetilde{\varphi}^{(s)}(n, x)=\left(\operatorname{Ad}_{u^{(s)}} \varphi^{(s)}\right)(n, x)=\widetilde{d}^{(s)}(x)+\widetilde{V}_{R}^{(s), 1}(n, x)+\widetilde{U}_{N R}^{(s)}(n, x)
$$

принадлежит $A^{\left(\rho_{s+1}\right)}$ и $\widetilde{d}^{(s)}(x), \widetilde{V}_{R}^{(s), 1}(n, x), \widetilde{U}_{N R}^{(s)}(n, x)$ - непрерывные функции вместе с их первыми двумя производными во всех расширениях $\mathrm{P} 3 \widetilde{O}_{t}^{(n)}, \bar{s} \leqslant t \leqslant s$. Кроме того, с помошью процедуры сглаживания, описанной в п. 3.1, мы можем представить $\widetilde{V}_{R}^{(s), 1}(n, x)$ в виде суммы двух слагаемых $\widetilde{U}_{R}^{(s), 1}(n, x)$ и $\widetilde{U}_{R}^{(s), 2}(n, x)$, каждое из которых непрерывно вместе с первыми двумя производньми в $\widetilde{O}_{\bar{s}}^{(n)}$, при этом $\widetilde{U}_{R}^{(s), 1}(n, x)=0$ для РЗ типа 3 и 4.

Теперь мы переходим к определению такого унитарного элемента $q^{(s)} \in A^{\left(\rho_{s+1}\right)}$, что $\operatorname{Ad}_{q^{(s)}}\left(\widetilde{U}_{R}^{(s), 2}\right)=0$.

Рассмотрим Р3 $O_{\bar{s}}^{(n)}$ и семейство двумерных операторов $K^{\bar{s}, n}(x)$, зависяших от параметра $x \in O_{s}^{(n)}$ :

$$
K^{\bar{s}, n}(x)=\left(\begin{array}{cc}
\widetilde{d}^{(s)}(x) & \widetilde{U}_{R}^{(s), 2}(n, x) \\
\widetilde{U}^{(s), 2}\left(-n, T^{n} x\right) & \widetilde{d}^{(s)}\left(T^{n} x\right)
\end{array}\right)
$$

Эта матрица симметрична, так как $\widetilde{\varphi}^{(s)}$ - самосопряженный элемент алгебры $A^{\left(\rho_{s+1}\right)}$. Тогда

$$
\lambda_{ \pm}^{\bar{s}, n}(x)=\frac{\widetilde{d}^{(s)}(x)+\widetilde{d}^{(s)}\left(T^{n} x\right)}{2} \pm \sqrt{\frac{\left(\widetilde{d}^{(s)}(x)-\widetilde{d}^{(s)}\left(T^{n} x\right)\right)^{2}}{4}+\left(\widetilde{U}_{R}^{(s), 2}(n, x)\right)^{2}}
$$

- собственные значения этой матрицы. Пусть

$$
Q^{(\bar{s}), n}(x)=\left(\begin{array}{rr}
\cos \Theta_{(\bar{s}), n}(x) & -\sin \Theta_{(\bar{s}), n}(x) \\
\sin \Theta_{(\bar{s}), n}(x) & \cos \Theta_{(\bar{s}), n}(x)
\end{array}\right)
$$

есть ортогональная матрица, приводящая $K^{\bar{s}, n}(x)$ к диагональному виду. Отметим, что $Q^{(\bar{s}), n}(x)$ отлична от единичной только для тех $x$, для которых $\widetilde{U}_{R}^{(s), 2}(n, x) \neq 0$. Функции $\lambda_{ \pm}^{\bar{s}, n}(x)$ непрерьвны вместе с их первыми и вторыми производными в $O_{\bar{s}}^{(n)}$ и совпадают с $\widetilde{d}^{(s)}(x), \widetilde{d}^{(s)}\left(T^{n} x\right)$ на границе $\mathrm{P} 3 O_{\bar{s}}^{(n)}$. Матрища $K^{\bar{s}, n}(x)$ может быть приведена к диагональному виду лишш с точностью до перестановки ее диагональных элементов. Для того чтобы обеспечить необходимую гладкость элементов матрицы $Q^{(\bar{s}), n}(x)$, мы определяем $Q^{(\bar{s}), n}(x)$ в зависимости от типа РЗ следуюшим образом.

1) Для РЗ типа 1 или 2. В этом случае функция $\widetilde{d}^{(s)}(x)$ монотонна на $O_{\bar{s}}^{(n)}$. Предположим, что $\widetilde{d}^{(s)}(x)$ возрастает как функция локальной координаты $x$; тогда мы выбираем $Q^{(\bar{s}), n}(x)$ таким образом, что

$$
Q^{(s), n}(x)^{*} K^{\bar{s}, n}(x) Q^{(s), n}(x)=\left(\begin{array}{cc}
\lambda_{1}^{\bar{s}, n}(x) & 0 \\
0 & \lambda_{2}^{\bar{s}, n}(x)
\end{array}\right),
$$


где $\lambda_{1}^{\bar{s}, n}(x)=\lambda_{-}^{\bar{s}, n}(x)$ для $x<x_{*}$ и $\lambda_{1}^{\bar{s}, n}(x)=\lambda_{+}^{\bar{s}, n}(x)$ для $x \geqslant x_{*}$. Если $\widetilde{d}^{(s)}(x)$ убьвает, положим $\lambda_{1}^{\bar{s}, n}(x)=\lambda_{+}^{\bar{s}, n}(x)$ для $x<x_{*}$ и $\lambda_{1}^{\bar{s}, n}(x)=\lambda_{-}^{\bar{s}, n}(x)$ для $x \geqslant x_{*}$. Ясно, что все элементы матрицы $Q^{(\bar{s}), n}(x)$ и $\lambda_{1}^{\bar{s}, n}(x)$ принадлежат $C^{2}\left(O_{\bar{s}}^{(n)}\right)$, так как в некоторой окрестности точки $x_{*}$ матрица $Q^{(\bar{s}), n}(x)$ совпадает с единичной.

2) Для Р3 $O_{\bar{s}}^{(n)}$ типа 3 матрица $Q^{(\bar{s}), n}(x)$ выбирается так, что $\lambda_{1}^{\bar{s}, n}(x)=\lambda_{+}^{\bar{s}, n}(x)$, если $\widetilde{d}^{(s)}(x)$ убывает в $O_{\bar{s}}^{(n)}, \lambda_{1}^{\bar{s}, n}(x)=\lambda_{-}^{\bar{s}, n}(x)$, если $\widetilde{d}^{(s)}(x)$ возрастает.

3) Для РЗ $O_{\bar{s}}^{(n)}$ типа 4 матрица $Q^{(\bar{s}), n}(x)$ выбирается таким образом, что $\lambda_{1}^{\bar{s}, n}(x)=$ $\lambda_{+}^{\bar{s}, n}(x)$, если $\widetilde{d}^{(s)}(x)$ имеет минимум в $O_{\bar{s}}^{(n)}, \lambda_{1}^{\bar{s}, n}(x)=\lambda_{-}^{\bar{s}, n}(x)$, если $\widetilde{d}^{(s)}(x)$ имеет максимум в $O_{\bar{s}}^{(n)}$.

ПРЕДЛОЖЕНИЕ 6. $\left(Q^{(\bar{s}), n}(x)\right)^{-1}=Q^{(\bar{s}), n}\left(T^{n} x\right)=\left(Q^{(\bar{s}), n}(x)\right)^{*}$.

Рассмотрим унитарньй элемент $q^{(s)}(n, x) \in A^{\left(\rho_{s+1}\right)}: q^{(s)}(0, x)=\cos \Theta_{(\bar{s}), n}(x)$, $q^{(s)}(n, x)=\sin \Theta_{(\bar{s}), n}(x), q^{(s)}(m, x)=0$ для $m \neq 0, n$ и для $x \in O_{\bar{s}}^{(n)}, q^{(s)}(0, x)=1$, $q^{(s)}(m, x)=0$ для $m \neq 0$, если $x \notin \bigcup O_{\bar{s}}^{(n)}$, где объединение берется по всем РЗ типа $1,2,3$ и 4 . Обозначим последнее через $O_{\bar{s}}$.

ПрЕДЛОЖЕНИЕ 7. Справедливо следующее равенство:

$$
\left(\operatorname{Ad}_{q^{(s)}}\left(\widetilde{\varphi}^{(s)}\right)\right)(n, x)=\tilde{\tilde{d}}^{(s)}(x)+\left(\operatorname{Ad}_{q^{(s)}}\left(\widetilde{U}_{R}^{(s), 1}\right)\right)(n, x)+\left(\operatorname{Ad}_{q^{(s)}}\left(\widetilde{U}_{N R}^{(s), 1}\right)\right)(n, x)
$$

где $\tilde{\tilde{d}}^{(s)}(x)=\widetilde{d}^{(s)}(x)$ для $x \notin O_{\bar{s}}, \tilde{\tilde{d}}^{(s)}(x)=\lambda_{1}^{\bar{s}, n}(x)$ для $x \in O_{\bar{s}}$. Кроме того, $\left\|q^{(s)}\right\|_{s+1}<C \varepsilon_{s+1}^{-1}$. Здесь $O_{\bar{s}}-$ обгединение РЗ порядка $\bar{s}$.

Функиия $\tilde{\tilde{d}}^{(s)}(x)$ имеет по крайней мере одну критическую точку на каждом интервале непрерывности.

ДоказАтЕльство. Равенства для $\tilde{\tilde{d}}^{(s)}(x)$ следуют непосредственно из определения элемента $q^{(s)}$. Норма

$$
\left\|q^{(s)}\right\|_{s+1} \leqslant \max _{x \in O_{\bar{s}}}\left(1,\left|\cos \Theta_{\bar{s}, n}(x)\right|+\left|\sin \Theta_{\bar{s}, n}(x)\right| e^{\rho_{s+1}|n|}\right) .
$$

Следовательно, $\left\|q^{(s)}\right\|_{s+1}<1+e^{\rho R_{\bar{s}}}$. Так как $R_{\bar{s}}=\left[\Gamma \bar{s}^{2} \ln \varepsilon_{\bar{s}}^{-1}\right]$, то

$$
e^{\rho R_{\bar{s}}} \leqslant \varepsilon_{\bar{s}}^{-\rho \Gamma \bar{s}^{2}} \leqslant \varepsilon_{s+1}^{-\rho \Gamma s^{2} /(1+\kappa)^{1+s-\bar{s}}} .
$$

Но $s+1-\bar{s}=g(s)+1$ и

$$
(1+\kappa)^{g(s)+1}>e^{\ln 10+\ln \Gamma \rho+2 \ln s}=10 \Gamma \rho s^{2} .
$$

Значит, $\left\|q^{(s)}\right\|_{s+1}<\varepsilon_{s+1}^{-1}$.

У тверждение о сушествовании по крайней мере одной критической точки на каждом интервале непрерьвности функции $\tilde{\tilde{d}}^{(s)}(x)$ вытекает непосредственно из определения этой функции для РЗ типа 3. 
ПРЕДЛОЖЕНИЕ 8.

$$
\begin{gathered}
\left\|\operatorname{Ad}_{q^{(s)}} \widetilde{U}_{N R}^{(s), 1}\right\|_{s+1}<\varepsilon_{s+1}^{4}, \\
\left|\frac{d \operatorname{Ad}_{q^{(s)}} \widetilde{U}_{N R}^{(s), 1}}{d x}\right|_{s+1}<\varepsilon_{s+1}^{3}, \quad\left\|\frac{d^{2} \operatorname{Ad}_{q^{(s)}} \widetilde{U}_{N R}^{(s), 1}}{d x^{2}}\right\|_{s+1}<\varepsilon_{s+1}^{2} .
\end{gathered}
$$

ДокАЗАТЕльСТВо. Оценки для нормы $\operatorname{Ad}_{q^{(s)}} \widetilde{U}_{N R}^{(s), 1}$ и ее производных вытекают из оценок, полученных в предложениях 5 и 7 , и неравенств

$$
\left\|\frac{d q^{(s)}}{d x}\right\|_{s+1}<\varepsilon_{s+1}^{-2}, \quad\left\|\frac{d^{2} q^{(s)}}{d x^{2}}\right\|_{s+1}<\varepsilon_{s+1}^{-3}
$$

( эти неравенства проверяются непосредственным вычислением производных, используя явные формулы для $\left.q^{(s)}(n, x)\right)$.

Теперь мы должны получить оценки для $\operatorname{Ad}_{q^{(s)}} \widetilde{U}_{R}^{(s), 1}(n, x)$. Прежде всего, заметим, что для РЗ типа 3 и $4 \operatorname{Ad}_{q^{(s)}} \widetilde{U}_{R}^{(s), 1}(n, x) \equiv 0$. Для того чтобы рассмотреть РЗ типа 1 и 2 , нам потребуются более точные оценки

$$
\left|q^{(s)}(n, x)\right|, \quad\left|\frac{d q^{(s)}(n, x)}{d x}\right| \text { и }\left|\frac{d^{2} q^{(s)}(n, x)}{d x^{2}}\right|
$$

для $x \in O_{\bar{s}}^{(n)}$.

Мы можем выписать явньй вид $\operatorname{Ad}_{q^{(s)}} \widetilde{U}_{R}^{(s), 1}(n, x)$ для $x \in O_{\bar{s}}^{(n)}$ :

$$
\operatorname{Ad}_{q^{(s)}} \widetilde{U}_{R}^{(s), 1}(m, x)= \begin{cases}\widetilde{U}_{R}^{(s), 1}(n, x) \sin 2 \Theta_{\bar{s}, n}(x) & \text { для } m=0, \\ \widetilde{U}_{R}^{(s), 1}(n, x) \cos 2 \Theta_{\bar{s}, n}(x) & \text { для } m=n, \\ 0 & \text { для всех остальных } m .\end{cases}
$$

Легко проверить, что

$$
\begin{aligned}
& \left|\sin 2 \Theta_{\bar{s}, n}(x)\right|=2\left|\frac{K(n, x)\left(\widetilde{d}^{(s)}(x)-\lambda_{1}^{\bar{s}, n}(x)\right)}{(K(n, x))^{2}+\left(\widetilde{d}^{(s)}(x)-\lambda_{1}^{\bar{s}, n}(x)\right)^{2}}\right|, \\
& \left|\cos 2 \Theta_{\bar{s}, n}(x)\right|=\left|\frac{K(n, x)^{2}-\left(\widetilde{d}^{(s)}(x)-\lambda_{1}^{\bar{s}}(x)\right)^{2}}{(K(n, x))^{2}+\left(\widetilde{d}^{(s)}(x)-\lambda_{1}^{\bar{s}, n}(x)\right)^{2}}\right|,
\end{aligned}
$$

где с целью сокрашения обозначений $\widetilde{U}_{R}^{(s), 1}(n, x)$ обозначено через $K(n, x)$.

Для РЗ $O_{\bar{s}}^{(n)}$ типа $1 \lambda_{1}^{\bar{s}, n}(x)$ отлично от $\widetilde{d}^{(s)}(x)$ только вне некоторой окрестности гранищы РЗ порядка $O_{\bar{s}+1}^{(n)}$ и, следовательно,

$$
\left|\widetilde{d}^{(s)}(x)-\widetilde{d}^{(s)}\left(T^{n} x\right)\right|>\varepsilon_{\bar{s}+1-g(\overline{s+1})}^{\mu} .
$$

Для РЗ типа 2

$$
\left|\widetilde{d}^{(s)}(x)-\widetilde{d}^{(s)}\left(T^{n} x\right)\right|>C \varepsilon_{s+1}^{1 / 2}\left(R_{\bar{s}}^{-(\nu+2)}\right) .
$$


В самом деле, точка $x^{*}$ находится от $x_{*}$ на расстоянии, большем $C \varepsilon_{s+1}^{1 / 2}$, так как

$$
\left|\widetilde{U}_{R}^{(s), 2}\left(n, x^{*}\right)-\widetilde{U}_{R}^{(s), 2}\left(n, x^{* *}\right)\right|>C \varepsilon_{s+1}, \quad\left|\frac{d \widetilde{U}_{R}^{(s), 2}(n, x)}{d x}\right|<C_{1} \varepsilon_{s+1}^{1 / 2}
$$

на отрезке $\left[x^{* *}, x^{*}\right]$, точка $x^{* *}$ лежит внутри отрезка $\left[x_{*}, x^{*}\right]$ и

$$
\left|\frac{d \widetilde{d}^{(s)}(x)}{d x}-\frac{d \widetilde{d}^{(s)}\left(T^{n} x\right)}{d x}\right|>C_{2} R_{\bar{s}}^{-(\nu+2)}
$$

$\left(\right.$ как это следует из $\left.5_{s}\right)$ и $6_{s}$ ) и оценок разности $d_{1}^{(s)}(x)-d^{(s)}(x)$ и ее производных).

Непосредственный подсчет показьвает, что

$$
\begin{gathered}
|A|<C \frac{\delta^{2}}{|a|}, \quad|B|<1-C \frac{\delta^{4}}{a^{2}} \\
\left|A^{\prime}\right|<C\left(\left|\frac{\delta^{\prime}}{a}\right|+\frac{\left|a^{\prime} \delta\right|}{a^{2}}\right), \quad\left|B^{\prime}\right|<C\left(\frac{\left|\delta \delta^{\prime}\right|}{a^{2}}+\frac{\left|a^{\prime} \delta^{2}\right|}{\left|a^{3}\right|}\right) \\
\left|A^{\prime \prime}\right|<C\left(\frac{\left|a^{\prime} \delta^{\prime}\right|}{a^{2}}+\frac{\left|a^{\prime \prime} \delta\right|}{a^{2}}+\frac{a^{\prime 2} \delta^{2}}{\left|a^{3}\right|}+\frac{\left|\delta^{\prime \prime}\right|}{|a|}+\frac{\left|\delta \delta^{\prime 2}\right|}{\left|a^{3}\right|}\right) \\
\left|B^{\prime \prime}\right|<C\left(\frac{\delta^{\prime 2}}{\left|a^{3}\right|}+\frac{\left|\delta \delta^{\prime}\right|}{\left|a^{3}\right|}+\frac{\left|a^{\prime} \delta\right|}{\left|a^{3}\right|}+\frac{\left|a^{\prime \prime} \delta^{2}\right|}{\left|a^{3}\right|}+\frac{a^{\prime 2} \delta^{2}}{a^{4}}\right)
\end{gathered}
$$

где с целью упрощения обозначений ' обозначает $\frac{d}{d x}$,

$A=\sin 2 \Theta_{\bar{s}, n}(x), \quad B=\cos 2 \Theta_{\bar{s}, n}(x), \quad a=\widetilde{d}^{(s)}(x)-\widetilde{d}^{(s)}\left(T^{n} x\right), \quad \delta=\widetilde{U}_{R}^{(s), 1}(n, x)$.

Используя эти оценки, легко убедиться в справедливости предложения 8.

Мы полагаем $\tau_{*}^{s}(x)=\bar{s}$ для всех $x$, для которых $q^{(s)}(n, x)$ отлично от единицы.

Теперь сформулируем основной результат п. 3.3 .

ПРЕДЛОЖЕНИЕ 9. Элемент

$$
\bar{\varphi}^{(s+1)}(n, x)=\left(q^{(s)^{*}} \cdot u^{(s)^{*}} \varphi^{(s)} \cdot u^{(s)} q^{(s)}\right)(n, x)
$$

может быть представлен в следующем виде:

$$
\bar{\varphi}^{(s+1)}(n, x)=\bar{d}^{(s+1)}(x)+\bar{V}_{R}^{(s+1)}(n, x)+\bar{V}_{N R}^{(s+1)}(n, x),
$$

где $\left\|\bar{V}_{N R}^{(s+1)}(n, x)\right\|_{s+1}<\varepsilon_{s+1}, \bar{V}_{R}^{(s+1)}(n, x)$ отлично от нуля только для РЗ порядка $t, \bar{s}+1 \leqslant t \leqslant s$, все функции $\bar{d}^{(s+1)}, \bar{V}_{R}^{(s+1)}, \bar{V}_{N R}^{(s+1)}$ непрерывны вместе $c$ их первыми и вторыми производными внутри расширения каждой из этих РЗ $O_{t}^{(n)}, \bar{s}+1 \leqslant t \leqslant s$,

$$
\begin{gathered}
\left\|\bar{V}_{R}^{(s+1)}(n, x)\right\|_{s+1}<C \varepsilon_{t_{1}^{(s)}(x)}, \\
\left\|\frac{d}{d x} \bar{V}_{R}^{(s+1)}(n, x)\right\|_{s+1}<C \varepsilon_{t_{1}^{(s)}(x)}^{3 / 4}, \quad\left|\frac{d^{2}}{d x^{2}} \bar{V}_{R}^{(s+1)}(n, x)\right|_{s+1} \leqslant C \varepsilon_{t_{1}^{(s)}(x)}^{1 / 2}
\end{gathered} .
$$

Кроме того, $\bar{V}_{R}^{(s+1)}(n, x) \equiv 0$ для РЗ $O_{\bar{s}+1}^{(n)}$, возникших из РЗ $O_{\bar{s}}^{(n)}$ mипа 3 $u 4, u$

для РЗ $O_{\bar{s}+1}^{(n)}$, возникших из РЗ типа 3.

$$
\left|\bar{d}^{(s+1)}(x)-\bar{d}^{(s+1)}\left(T^{n} x\right)\right| \geqslant \frac{1}{16} \varepsilon_{s+1}
$$


ДокАЗАТЕльство. Мы полагаем $V_{N R}^{(s+1)}(n, x)=\bar{\varphi}^{(s+1)}(n, x)$ вне РЗ порядка $t$, $\bar{s}+1 \leqslant t \leqslant s, V_{R}^{(s+1)}(n, x)=0$ вне этих РЗ. Неравенство

$$
\left|\bar{d}^{(s+1)}(x)-\bar{d}^{(s+1)}\left(T^{n} x\right)\right| \geqslant \frac{1}{16} \varepsilon_{s+1}
$$

следует для РЗ типа 3 из того, что

$$
\bar{d}^{(s+1)}(x)=\lambda_{1}^{(n, \bar{s})}(x), \bar{d}^{(s+1)}\left(T^{n} x\right)=\lambda_{1}^{(n, \bar{s})}\left(T^{n} x\right)
$$

И

$$
\left|\bar{d}^{(s+1)}(x)-\bar{d}^{(s+1)}\left(T^{n} x\right)\right|=\left|\lambda_{+}^{(n, \bar{s})}(x)-\lambda_{-}^{(n, \bar{s})}(x)\right| \geqslant \frac{1}{16} \varepsilon_{s+1} .
$$

ЗАМЕЧАНИЕ. Отметим, что из РЗ типа 3 и порядка $\bar{s}$ образовались РЗ типа 4 и порядка $\bar{s}+1$. В этих зонах между значениями $\bar{d}^{(s+1)}(x)$ и $\bar{d}^{(s+1)}\left(T^{n} x\right)$ возникла шель порядка $\varepsilon_{s+1}$. Как легко видеть, из РЗ типа 4 и порядка $\bar{s}$ образовались Р3 того же типа и порядка $\bar{s}+1$, и для них аналогичная щель больше $\frac{1}{16} \varepsilon_{s}-C \varepsilon_{s+1}$. Это означает, что образовавшиеся Р3 типа 4 останутся Р3 того же типа на последуюших шагах индукции до тех пор, пока щель порядка $\varepsilon_{s}$ не станет больше, чем $\varepsilon_{s+k-g(s+k)}^{\mu}$. После этого на последуюших шагах эта область перестанет быть РЗ, и легко проверить, что $k$ не превосходит $C s$.

Итак, мы получили элемент $\bar{\varphi}^{(s+1)}(n, x)$, для которого вьполняются все индуктивные предположения для РЗ порядка $t$, где $\bar{s}+1 \leqslant t \leqslant s$, и их сдвигов на $n$, где $|n| \leqslant R_{s}$. Для того чтобы получить элемент $\varphi^{(s+1)}(n, x)$, удовлетворяюший всем индуктивньм предложениям $\left.\left.0_{s+1}\right)-6_{s+1}\right)$, необходимо рассмотреть РЗ порядка $(s+1)$ и проверить вьполнение остальных индуктивных предположений. Это будет сделано в следующем пункте.

3.4. Геометрия резонансных зон. Анализ разрывов. Окончание доказательства индуктивной леммы. Мы видели в предыдушем разделе, что функции $\bar{d}^{(s+1)}(x), \bar{V}_{R}^{(s+1)}(n, x), \bar{V}_{N R}^{(s+1)}(n, x)$ непрерьвны во всех расширениях $\widetilde{O}_{t}^{(n)}, \overline{s+1} \leqslant$ $t \leqslant s$, РЗ типа 0,1 и 2 . Кроме того, эти функщии непрерывны в расширениях РЗ $\widetilde{O}_{\bar{s}}^{(n)}$ типа 4 , так как в этом случае каждая из функций $\bar{d}^{(s+1)}(x)$ и $\bar{d}^{(s+1)}\left(T^{n} x\right)$ имеет ровно одну критическую точку внутри РЗ $\widetilde{O}_{\bar{s}}^{(n)}(x)$ и $\widetilde{O}_{s}^{(-n)}\left(T^{n} x\right)$, соответственно (одна из этих точек - максимум, а другая - минимум); разность между критическими значениями этих функций больше $C \varepsilon_{s+1}, \mathrm{a} V_{R}^{(s), 1}(n, x)$ не превосходит $C \varepsilon_{\bar{s}}$ для всех $x \in O_{\bar{s}}^{(n)}$, так что разность $\bar{V}_{R}^{(s+1)}(n, x)-V_{R}^{(s), 1}(n, x)$ не превосходит по модулю $C \varepsilon_{s+1}^{2} \varepsilon_{\bar{s}}^{-1}$. Последнее означает, что разность между критическими значениями функции $\bar{d}^{(n+1)}(x)$ в $O_{\bar{s}}^{(n)}(x)$ и $O_{\bar{s}}^{(-n)}\left(T^{n} x\right)$ по модулю остается больше $C_{1} \varepsilon_{s+1}$, где $C_{1}>C\left(1-\varepsilon_{s+1} \varepsilon_{\bar{s}}^{-1}\right)$. На границах РЗ $O_{\bar{s}}^{(n)}$ типа 3 возникают разрьвы функций $\bar{d}^{(s+1)}(x)$ и $\bar{V}^{(s), 1}(n, x)$.

ПРЕДЛОЖЕНИЕ 10. Пусть $O_{\bar{s}}^{(n)}$ - PЗ типа 3 , содержащ, ая точку $x$, и $O_{\bar{s}}^{(-n)}$ - такая же РЗ, содержащая точку $T^{n} x$. Если $\bar{x}$ - точка разрьва функиии $\bar{d}^{(s+1)}(x)$, то предельные значения функций $\bar{d}^{(s+1)}(x)$ и $\bar{V}^{(s), 1}(m, x)$ и их производных при стремлении $x \kappa \bar{x}$ изнутри (извне) $Р 3 O_{\bar{s}}^{(n)}$ совпадают с предельнымми значениями функций $\bar{d}^{(s+1)}\left(T^{n}(x)\right)$ u $\bar{V}^{(s+1)}\left(m, T^{n}(x)\right)$ и их производных 
при стремлении $x \kappa \bar{x}$ изнутри (извне) РЗ $O_{\bar{s}}^{(n)}$. Функиия $\bar{d}^{(s+1)}(x)$ принимает каждое свое значение ровно два раза (с учетом кратности).

Доказательство непосредственно следует из определения функций $\bar{d}^{(s+1)}(x)$, $\bar{V}^{(s+1)}(x)$ и определения элемента $q^{(s)}(n, x)$. Заметим, что $|n| \leqslant R \bar{s}$.

Точно таким же образом возникали разрывы функции $d^{(t)}(x), t \leqslant s$, на предыдущих шагах индукщии при полном расщеплении РЗ. Используя это обстоятельство, мы покажем, что функции $\bar{d}^{(s+1)}(x), \bar{V}^{(s+1)}(x)$ могут быть переклеены таким образом, что будут выполнены индуктивные предположения $\left.\left.0_{s+1}\right)-6_{s+1}\right)$, причем эта переклейка будет осуществлена при помоши отображения $S^{1} \rightarrow S^{1}$, сохраняющего меру Лебега. Свойства этого отображения будут сформулированы в предложении 12 .

ПРЕДЛОЖЕНИЕ 11. Предположим, что на интервале

$$
\bar{B}_{n}^{(s+1)}=\left\{x:\left|\bar{d}^{(s+1)}(x)-\bar{d}^{(s+1)}\right|<2 \delta_{s+1}\right\},
$$

$R_{s}<|n| \leqslant R_{s+1}$, функиии $\bar{d}^{(s+1)}$ и $\bar{d}^{(s+1)}\left(T^{n} x\right)$ непрерывны $и$

$$
\lim _{x \rightarrow x_{ \pm}}\left|\bar{d}^{(s+1)}(x)-\bar{d}^{(s+1)}\left(T^{n} x\right)\right|=2 \delta_{s+1},
$$

где $x_{-}, x_{+}-$концы интервала $\bar{B}_{n}^{(s+1)}$ и $x \in \bar{B}_{n}^{(s+1)}$. Тогда справедливь следующие утверждения:

1) $C_{1} \delta_{s+1}<\left|\bar{B}_{n}^{(s+1)}\right|<C_{2} \delta_{s+1}^{1 / 2}$

2) существует такая точка $x_{*} \in \bar{B}_{n}^{(s+1)}$, что $\bar{d}^{(s+1)}\left(x_{*}\right)=\bar{d}^{(s+1)}\left(T^{n} x_{*}\right)$;

3) если $x^{*}$ - критическая точка функиии $\bar{d}^{(s+1)}(x)$, mо $\operatorname{dist}\left(x^{*}, \bar{B}_{n}^{(s+1)}\right)>$ $\delta_{s+1}^{1 / 3}$

Кроме того, пересечение $\widetilde{O}_{t}^{(m)} \cap \bar{B}_{n}^{(s+1)}$ пусто для $\overline{s+1} \leqslant t<s+1$ и любых $m$.

ДокАЗАТЕЛЬСтво. Из построения функции $\bar{d}^{(s+1)}(x)$ следует, что $\left|\frac{d \bar{d}^{(s+1)}(x)}{d x}\right|$ $<C$. Поэтому $\left|\bar{B}_{n}^{(s+1)}\right|>C_{1} \delta_{s+1}$.

Пусть $x^{*}-$ критическая точка функции $\bar{d}^{(s+1)}(x)$, принадлежашая интервалу непрерьвности функции $\bar{d}^{(s+1)}(x)$, содержащему $\bar{B}_{n}^{(s+1)}$. (В силу индуктивных предположений и определения функции $\bar{d}^{(s+1)}(x)$ такой интервал существует.) Предположим, что для некоторой точки $\bar{x} \in \bar{B}_{n}^{(s+1)}$ вьполняется $\left|\bar{d}^{(s+1)}\left(x^{*}\right)-\bar{d}^{(s+1)}(\bar{x})\right|<\delta$. Тогда $\left|\bar{d}^{(s+1)}\left(x^{*}\right)-\bar{d}^{(s+1)}\left(T^{n} \bar{x}\right)\right|<\delta+2 \delta_{s+1}$, т.е. $T^{n} \bar{x}$ принадлежит $C_{1}\left(\delta+2 \delta_{s+1}\right)^{1 / 2}$ окрестности точки $x^{*}$. Той же окрестности принадлежит и $\bar{x}$. Следовательно,

$$
\begin{aligned}
& 2 C_{1}\left(\delta+2 \delta_{s+1}\right)^{1 / 2}>\frac{\varepsilon^{\sigma}}{(s+1)^{2} \mathbb{R}_{s+1}^{\nu+1}} \\
& 2 C_{1}\left(\delta+2 \delta_{s+1}\right)^{1 / 2}>\frac{\varepsilon^{\sigma / 2}}{\left(\Gamma(s+1)^{2}(1+\kappa)^{s+1}\right)^{\nu+1}(s+1)^{2}}
\end{aligned}
$$


Значит,

$$
\delta+2 \delta_{s+1}>C_{3} \frac{\varepsilon^{\sigma / 2}}{\left((1+\kappa)^{2(\nu+1)}\right)^{s+1}} .
$$

Последнее неравенство не может быть верным, если $\delta<\delta_{s+1}^{1 / 3}$. Мы получаем, что $\bar{x}$ отстоит от $x^{*}$, по крайней мере, на расстояние $\delta_{s+1}^{1 / 3}$ и $\left|\frac{d \bar{d}^{(s+1)}(x)}{d x}\right| \geqslant C \delta_{s+1}^{1 / 3}$ для $x \in \bar{B}_{n}^{(s+1)}$. Такое же неравенство справедливо для $\frac{d \bar{d}^{(s+1)}\left(T^{n} x\right)}{d x}$.

Покажем теперь, что

$$
\operatorname{sgn} \frac{d \bar{d}^{(s+1)}(x)}{d x}=-\operatorname{sgn} \frac{d \bar{d}^{(s+1)}\left(T^{n} x\right)}{d x}
$$

для $x \in \bar{B}_{n}^{(s+1)}$. Для этого, прежде всего, заметим, что если $x_{1} \neq x_{2}$ и $\bar{d}^{(s+1)}\left(x_{1}\right)=$ $\bar{d}^{(s+1)}\left(x_{2}\right)$, то

$$
\left.\operatorname{sgn} \frac{d \bar{d}^{(s+1)}(x)}{d x}\right|_{x_{1}}=-\left.\operatorname{sgn} \frac{d \bar{d}^{(s+1)}(x)}{d x}\right|_{x_{2}}
$$

Предположим, что знаки производных совпадают и $x_{1}^{*}$ и $x_{2}^{*}-$ критические точки функции $\bar{d}^{(s+1)}(x)$ такие, что пары $\left(x_{1}, x_{1}^{*}\right)$ и $\left(x_{2}, x_{2}^{*}\right)$ принадлежат отрезку непрерьвности функции $\bar{d}^{(s+1)}(x)$ (такие критические точки сушествуют согласно индуктивным предположениям и определению функции $\left.\bar{d}^{(s+1)}(x)\right)$. Если

$$
\left(\bar{d}^{(s+1)}\left(x_{1}\right)-\bar{d}^{(s+1)}\left(x_{1}^{*}\right)\right)\left(\bar{d}^{(s+1)}\left(x_{2}\right)-\bar{d}^{(s+1)}\left(x_{2}^{*}\right)\right)>0,
$$

то при $\bar{d}^{(s+1)}\left(x_{1}^{*}\right)>\bar{d}^{(s+1)}\left(x_{2}^{*}\right)$ найдется такое $\bar{d},\left(\bar{d}-\bar{d}^{(s+1)}\left(x_{1}\right)\right)\left(\bar{d}-\bar{d}^{(s+1)}\left(x_{1}^{*}\right)\right)<0$, что $\bar{d}^{(s+1)}(x)$ принимает значение $\bar{d}$ более, чем в двух различных точках. Случай $\bar{d}^{(s+1)}\left(x_{1}^{*}\right)<\bar{d}^{(s+1)}\left(x_{2}^{*}\right)$ рассматривается аналогично.

Предположим теперь, что

$$
\left(\bar{d}^{(s+1)}\left(x_{1}\right)-\bar{d}^{(s+1)}\left(x_{1}^{*}\right)\right)\left(\bar{d}^{(s+1)}\left(x_{2}\right)-\bar{d}^{(s+1)}\left(x_{2}^{*}\right)\right)<0 .
$$

Мы покажем, что и в этом случае функция $\bar{d}^{(s+1)}(x)$ принимает некоторое значение по крайней мере в трех различных точках, что невозможно.

Рассмотрим несколько случаев.

1) Существует критическая точка $x_{1}^{* *}$ функции $\bar{d}^{(s+1)}(x)$ такая, что на интервале с концами $x_{1}^{* *}$ и $x_{1}^{*}$, содержашем $x_{1}$, функция $\bar{d}^{(s+1)}(x)$ непрерьвна. Из условия совпадения знаков производных $\frac{d \bar{d}^{(s+1)}(x)}{d x}$ в точках $x_{1}$ и $x_{2}$ следует, что $x_{1}^{* *} \neq x_{2}^{*}$. Тогда сушествует такое $\bar{d}$, близкое к $\bar{d}^{(s+1)}\left(x_{2}^{*}\right)$ при $\bar{d}^{(s+1)}\left(x_{2}^{*}\right)>\bar{d}^{(s+1)}\left(x_{1}^{* *}\right)$ или близкое к $\bar{d}^{(s+1)}\left(x_{1}^{* *}\right)$ при $\bar{d}^{(s+1)}\left(x_{2}^{*}\right)<\bar{d}^{(s+1)}\left(x_{1}^{* *}\right)$, что $\bar{d}^{(s+1)}(x)$ принимает значение $\bar{d}$ по крайней мере в трех различных точках. 
2) Сушествует точка $x_{1}^{\prime}$, являюшаяся точкой разрыва функции $\bar{d}^{(s+1)}(x)$, такая, что $\bar{d}^{(s+1)}(x)$ непрерьвна на интервале с концами $x_{1}^{\prime}$ и $x_{1}^{*}$, содержащем $x_{1}$ и не содержашем критических точек. Тогда из индуктивных предположений и определения функции $\bar{d}^{(s+1)}(x)$ легко вывести, что существует такое $p,|p|<R \frac{}{s+1}$, что $\overline{\bar{d}}^{(s+1)}(x)=$ $\bar{d}^{(s+1)}(e x)$, где $e$ - отображение окружности, переводящее некоторьй интервал (или полуинтервал) $I^{(s+1)}$ с конщом в $x_{1}^{\prime}$ в $T^{p} I^{(s+1)}$, а $I_{1}^{(s+1)}=T^{p} I^{(s+1)}$ в $T^{-p} I_{1}^{(s+1)}$, будет удовлетворять 1$)$. Следовательно, и в этом случае функция $\bar{d}^{(s+1)}(x)$ будет принимать некоторое свое значение по крайней мере в трех различных точках, что невозможно.

Итак, мы получаем, что

$$
\left.\operatorname{sgn} \frac{d \bar{d}^{(s+1)}(x)}{d x}\right|_{x_{1}}=-\left.\operatorname{sgn} \frac{d \bar{d}^{(s+1)}(x)}{d x}\right|_{x_{2}}
$$

Покажем теперь, что существует такая точка $x_{*} \in \bar{B}^{(s+1)}$, что

$$
\bar{d}^{(s+1)}\left(x_{*}\right)=\bar{d}^{(s+1)}\left(T^{n} x_{*}\right) .
$$

В самом деле, функции $\bar{d}^{(s+1)}(x)$ и $\bar{d}^{(s+1)}\left(T^{n} x\right)$ непрерьвны на замыкании $\bar{B}_{n}^{(s+1)}$ и, следовательно, непрерьвна разность $\bar{d}^{(s+1)}(x)-\bar{d}^{(s+1)}\left(T^{n} x\right)$. Модуль этой разности принимает одинаковые значения в концах интервала $\bar{B}_{n}^{(s+1)}$. Если сама разность принимает противоположные значения, то существование точки $x_{*}$ очевидно. Если же разность принимает в концах $\bar{B}_{n}^{(s+1)}$ одинаковые значения, то существует критическая точка этой разности внутри $\bar{B}_{n}^{(s+1)}$. Это означает, что производные $\frac{d \bar{d}^{(s+1)}(x)}{d x}$ и $\frac{d \bar{d}^{(s+1)}\left(T^{n} x\right)}{d x}$ имеют одинаковые знаки для $x \in \bar{B}_{n}^{(s+1)}$, так как $\bar{B}_{n}^{(s+1)}$ не содержит критических точек функций $\bar{d}^{(s+1)}(x)$ и $\bar{d}^{(s+1)}\left(T^{n} x\right)$, как было показано в начале доказательства. Из этого следует, что $\bar{d}^{(s+1)}\left(x_{1}\right) \neq \bar{d}^{(s+1)}\left(T^{n} x_{2}\right)$ для любых $x_{1}, x_{2} \in \bar{B}_{n}^{(s+1)}$. Таким образом, области значений функций $\bar{d}^{(s+1)}(x)$ и $\bar{d}^{(s+1)}\left(T^{n} x\right)$ для $x \in \bar{B}_{n}^{(s+1)}$ не пересекаются. Так как область значений каждой из этих функций находится в $2 \delta_{s+1}$-окрестности области значений другой и каждая из них отделена от значений в любой критической точке $x^{*}$ на расстояние, большее $\delta_{s+1}^{1 / 3}$, найдутся точки $\bar{x}_{1}$ и $\bar{x}_{2}$, в которых производные функции $\bar{d}^{(s+1)}(x)$ имеют одинаковьй знак, $\bar{d}^{(s+1)}\left(\bar{x}_{1}\right)=\bar{d}^{(s+1)}\left(\bar{x}_{2}\right)$ и одна из них принадлежит $\bar{B}_{n}^{(s+1)}$. Это невозможно.

Таким образом, нами доказано существование точки $x_{*} \in \bar{B}_{n}^{(s+1)}$, для которой $\bar{d}^{(s+1)}\left(x_{*}\right)=\bar{d}^{(s+1)}\left(T^{n} x_{*}\right)$. Из того, что $\bar{d}^{(s+1)}\left(x_{*}\right)$ и $\bar{d}^{(s+1)}\left(T^{n} x_{*}\right)$ имеют производные разных знаков при $x \in \bar{B}_{n}^{(s+1)}$ и каждая из них по модулю $\geqslant C \delta_{s+1}^{1 / 3}$, следует, что диаметр $\bar{B}_{n}^{(s+1)}$ не превосходит $\frac{2 \delta_{s+1}}{C \delta^{1 / 3}} \leqslant C \delta_{s+1}^{1 / 2}$. 
ПРЕДЛОЖЕНИЕ 12. Существует такое взаимно однозначное сохраняющее меру Лебега отображсение $e^{(s)}: S^{1} \rightarrow S^{1}$, что для әлемента

$$
\varphi^{(s+1)}(n, x)=\bar{\varphi}\left(e^{(s)}(x)\right)=d^{(s+1)}(x)+V^{(s+1)}(n, x)
$$

функции $d^{(s+1)}(x)$ и $V^{(s+1)}(x)$ непрерывны в расширении $\widetilde{O}_{s+1}^{(n)}$ любой РЗ порядка $s+1, R_{s}<|n| \leqslant R_{s+1}$.

Отображсние $e^{(s)}$ обладает следующими свойствами:

1) $e^{(s)}$ тохсдественно вне $\bigcup_{j} I_{j}, j \in J$, где $J$ - конечное мнохсество индексов, а каждое множество $I_{j}$ является интервалом или полуинтервалом;

2) $I_{j_{1}} \cap I_{j_{2}}=\varnothing$ npu $j_{1} \neq j_{2}$;

3) для каждого $j_{1}$ существует одно и только одно $j_{2}$ такое, что $I_{j_{2}}=$ $T^{p\left(j_{1}\right)} I_{j_{1}} u e^{(s)}(x)=T^{p(j)} x$ для $x \in I_{j_{1}}, p\left(j_{2}\right)=-p\left(j_{1}\right),\left|p\left(j_{1}\right)\right|<R_{\bar{s}}$

4) хотя бы одно из множеств $I_{j_{1}}, I_{j_{2}}=T^{p\left(j_{1}\right)} I_{j_{1}}$ принадлежст расширению $\widetilde{O}_{s+1}^{n\left(j_{1}\right)} P 3$ порядка $s+1 ;$

5) $I_{j} \cup O_{t}^{(n)}=\varnothing$ для всех $j \in J u \overline{s+1} \leqslant t \leqslant s$;

6) мера Лебега $\mu\left(I_{j}\right)<C \delta_{s}^{1 / 2}$ для всех $j \in J$.

ДоКАЗАТЕльство непосредственно вытекает из следуюших утверждений.

1) Пусть $\widetilde{O}_{s+1}^{\left(n_{1}\right)}$ и $\widetilde{O}_{s+1}^{\left(n_{2}\right)}$ - расширения двух РЗ порядка $s+1$, принадлежаших одному участку монотонности функщии $\bar{d}^{(s+1)}(x)$, заключенному между двумя критическими точками $x_{1}^{*}$ и $x_{2}^{*}$. Тогда $\operatorname{dist}\left(\widetilde{O}_{s+1}^{\left(n_{1}\right)}, \widetilde{O}_{s+1}^{\left(n_{2}\right)}\right)>C_{1} \delta_{s+1}^{1 / 3}$.

В самом деле, согласно предложению 11 сушествуют такие точки $x_{1} \in \widetilde{O}_{s+1}^{\left(n_{1}\right)}$ и $x_{2} \in \widetilde{O}_{s+1}^{\left(n_{2}\right)}$, что $\bar{d}^{(s+1)}\left(x_{1}\right)=\bar{d}^{(s+1)}\left(T^{n_{1}} x_{1}\right)$ и $\bar{d}^{(s+1)}\left(x_{2}\right)=\bar{d}^{(s+1)}\left(T^{n_{1}} x_{2}\right)$. Кроме того, $\operatorname{diam} \widetilde{O}_{s+1}^{\left(n_{1}\right)}<C \delta_{s+1}^{1 / 2}$ и $\operatorname{diam} \widetilde{O}_{s+1}^{\left(n_{2}\right)}<C \delta_{s+1}^{1 / 2}$. Тогда

$$
\begin{aligned}
& \left|\bar{d}^{(s+1)}\left(T^{n_{1}} x_{2}\right)-\bar{d}^{(s+1)}\left(T^{n_{2}} x_{2}\right)\right| \\
& \quad<\left|\bar{d}^{(s+1)}\left(T^{n_{1}} x_{1}\right)-\bar{d}^{(s+1)}\left(T^{n_{1}} x_{2}\right)\right|+\left|\bar{d}^{(s+1)}\left(T^{n_{1}} x_{1}\right)-\bar{d}^{(s+1)}\left(T^{n_{2}} x_{2}\right)\right| \\
& \quad<2 C\left|x_{1}-x_{2}\right|<C_{2} \delta_{s+1}^{1 / 3},
\end{aligned}
$$

если $\left|x_{1}-x_{2}\right|<\delta_{s+1}^{1 / 3}$. С другой стороны, можно показать, что

$$
\left|\bar{d}^{(s+1)}\left(T^{n_{1}} x_{2}\right)-\bar{d}^{(s+1)}\left(T^{n_{2}} x_{2}\right)\right|>C \delta_{s+1}^{1 / 6}\left|\left\{\left(n_{1}-n_{2}, \omega\right)\right\}\right| .
$$

Имеем $\left|\left\{\left(n_{1}-n_{2}, \omega\right)\right\}\right|<C_{4} \delta_{s+1}^{1 / 6}$, что невозможно при $\left|n_{1}\right|,\left|n_{2}\right|<R_{s+1}$ и диофантовом условии $(D)$.

2) Так как $\operatorname{diam}\left(\widetilde{O}_{s+1}^{\left(n_{1}\right)}\right)<C \delta_{s+1}^{1 / 2}$, можно для любой точки разрьва $\bar{x}$ найти такой интервал или полуинтервал $\Delta(\bar{x})$ с концом в $\bar{x}$, что длина этого интервала не превосходит $C \delta_{s+1}^{1 / 2}$, и сушествует такое $p=p(\bar{x}),|p|<R_{s+1}$, что функшия $d^{(s+1)}(x)$, равная $\bar{d}^{(s+1)}(x)$ в одной полуокрестности точки $\bar{x}$ и $d^{(s+1)}\left(T^{-p} y\right)$ для $y \in \Delta(\bar{x})$ в другой ее полуокрестности, будет непрерывна в $\widetilde{O}_{s+1}^{(n)}$. Тогда преобразование $e^{(s)}(x)=$ 
$T^{-p(\bar{x})} x$ для $x \in \Delta(\bar{x}), e^{(s)}(x)=T^{p(\bar{x})} x$ для $x \in T^{-p(\bar{x})} \Delta(\bar{x}), e^{(s)}(x)$ тождественно вне $\bigcup_{\bar{x}} \Delta(\bar{x})$, где объединение берется по всем точкам разрьва $\bar{x}$, принадлежащим множеству

$$
\left\{x:\left|\bar{d}^{(s+1)}(x)-\bar{d}^{(s+1)}\left(T^{n} x\right)\right|<2 \delta_{s+1}, R_{s}<|n| \leqslant R_{s+1}\right\},
$$

есть искомое преобразование. Из пункта 1) следует, что это преобразование корректно определено.

Положим $\tau^{(s)}(x), \tau_{1}^{(s)}(x)$ и $\tau_{*}^{(s)}(x)$ равными $\tau^{(s)}\left(e^{(s)^{-1}}(x)\right), \tau_{1}^{(s)}\left(e^{(s)^{-1}}(x)\right)$ и $\tau_{*}^{(s)}\left(e^{(s)^{-1}}(x)\right)$, соответственно.

Остальные индуктивные предположения проверяются непосредственно.

\section{СПИСОК ЛИТЕРАТУРЫ}

[1] Sinai Ya. Anderson localization for one-dimensional difference Schrödinger operator with quasi-periodic ponential // J. Statist. Phys. 1987. V. 46. P. 861-909.

[2] Chulaevsky V.A., Sinai Ya. G. Anderson localization for the 1-D discrete Schrödinger operator with two-frequency potential // Comm. Math. Phys. 1989. V. 125. P. 91.

[3] Chulaevsky V.A., Sinai Ya. G. Anderson localization and KAM theory // Analysis, et Cetera. New York: Academic Press, 1990.

[4] Chulaevsky V.A., Sinai Ya. G. The exponential localization and structure of the spectrum for 1D quasi-periodic discrete Schrödinger operators // Rev. Math. Phys. 1991. V. 3. № 3. Р. 241-284.

[5] Динабург Е. И., Синай Я. Г. Об одномерном уравнении Шрёдингера с квазипериодическим потенциалом // Функц. анал. и прилож. 1975. Т. 9. С. 8-21.

[6] Carmona R., Lacroix J. Spectral theory of random Schrödinger operators. Boston: Birkhäuser, 1990.

[7] Aizenman M., Molchanov S. Localization at large disorder and at extreme energies: An elementary deviation // Comm. Math. Phys. 1993. V. 157. P. 245-278.

[8] Корнфельд И.П., Синай Я.Г., Фомин С.В.Эргодическая теория. М.: Наука, 1980.

[9] Chulaevsky V. A., Spenser T. Positive Lyapunov exponents for a class of deterministic potentials // Comm. Math. Phys. 1995. V. 168. P. 455-466.

[10] Пастур Л.А., Фиготин А. Локализация для одного несоизмеримого потенциала: Точно решаемая многомерная модель // Письма ЖЭЭФ. 1983. Т. 37. С. 686-688.

[11] Simon B. Almost periodic Schrodinger operators. IV. Maryland model // Ann. Phys. 1985. V. 159. P. $157-183$.

[12] Bellissard J., Lima R., Scoppola E. Localization in $\nu$-dimensional incommesurate media // Comm. Math. Phys. 1983. V. 88. P. 465-477.

[13] Frohlich J. Spencer T., Wittwer P. Localization for a class of one-dimensional quasi-periodic Schrödinger operator // Comm. Math. Phys. 1990. V. 132. P. 5-25.

[14] Jitomirskaya S., Simon B. Operator with singular continuous spectrum: III Almost periodic Schrodinger operators // Comm. Math. Phys. 1994. V. 165. P. 201-205.

[15] Avron J., Simon B. Almost periodic Schrodinger operators, II. The integrated density of states // Duke Math. J. 1983. V. 50. P. 369-391.

[16] Herman M. Une methode pour minorer les exposant de Lyapunov et exemples montrant le caractere local d'un theoreme d'Arnold et de Moser sur le tore en dimension 2 // Comment. Math. Helv. 1983. V. 58. P. 453-502.

[17] Jitomirskaya S. Anderson localization for the almost Mathieu equation: A nonperturbative proof // Comm. Math. Phys. 1994. V. 165. P. 49-57; II Point spectrum for $\lambda>2 / /$ Comm. Math. Phys. 1995. V. 168. P. 563-570.

[18] Gesztesy F., Simon B. The xi function // Preprint, 1995. 
[19] Avron F., Simon B. Singular continuous spectrum for a class of almost periodic Jacobi matrices // Bull. Amer. Math. Soc. (N.S.). 1982. V. 6. P. 81-85.

[20] Last Y. A relation between absolutely continuous spectrum of ergodic Jacobi matrices and the spectra of periodic approximants // Comm. Math. Phys. 1993. V. 151. P. 183-192.

[21] Aubry S., Andre G. Analyticity breaking and Anderson localzation in incommensurate lattices // Ann. Israel Phys. Soc. 1981. V. 3. P. 133-164.

[22] Thouless D. J. Bandwidth for a quasiperiodic tight binding model // Phys. Rev. 1982. V. B28. P. 4272-4276.

[23] Avron F., van Mouche P., Simon B. On the measure of the spectrum for the almost Mathieu operator // Comm. Math. Phys. 1990. V. 132. P. 103-118.

[24] Eliasson L. Floquet solution for 1-dimensional quasi-periodic Schrödinger equation // Comm. Math. Phys. 1992. V. 146. P. 447-482.

[25] Johnson R., Moser J. The rotation number for almost periodic potentials // Comm. Math. Phys. 1982. V. 84. P. 403-438.

[26] Белоколос Е. Д. Квантовая частица в однородной деформированной решетке. Оценки размера лакун в спектре // ТМФ. 1975. Т. 25. № 3. С. 344-357.

[27] Moser J., Poschel J. An extension of a result by Dinaburg and Sinai on quasi-periodic potentials // Comm. Math. Helvetici. 1984. V. 59. P. 39-85.

[28] Chulaevsky V. A., Dinaburg E. I. Methods of KAM-theory for long-range quasi-periodic operators on $\mathbb{Z}^{\nu}$. Pure point spectrum // Comm. Math. Phys. 1993. V. 153. P. 559-577.

[29] Gordon A., Jitomirskaya S., Last Y., Simon B. Duality and Singular Continuos Spectrum in the Almost Mathieu Equations // Preprint, 1996.

Объединенный институт физики Земли им. О. Ю. Шмидта РАН

Поступила в редакцию

31.07.1996 